\title{
The ammonoids from the Dalle à Merocanites of Timimoun (Late Tournaisian-Early Viséan; Gourara, Algeria)
}

\author{
Volker Ebbighausen $^{1}$, Dieter Korn ${ }^{*}, 2$ and Jürgen Bockwinkel ${ }^{3}$ \\ ${ }^{1}$ Engstenberger Höhe 12, 51519 Odenthal, Germany. E-mail: Volker@vxr.de \\ ${ }^{2}$ Museum für Naturkunde Berlin, Invalidenstraße 43, 10115 Berlin, Germany. E-mail: dieter.korn@mfn-berlin.de \\ ${ }^{3}$ Dechant-Feinstraße 22, 51375 Leverkusen, Germany. E-mail: jbockwinkel@t-online.de
}

\begin{abstract}
Received 11 May 2009

Accepted 26 June 2009

Published 19 February 2010

\section{Key Words}

\section{Ammonoidea}

Mississippian

Carboniferous

North Africa

taxonomy

The ammonoids from the Early Carboniferous (Mississippian) Dalle à Merocanites of Timimoun (Gourara, Algeria) are described. The following new ammonoid taxa are introduced: subfamily Hammatocyclinae n. subfam., Hammatocyclus pollex n. sp., Hammatocyclus corrugatus n. sp., Neopericyclus arenosus n. sp., Ammonellipsites pareyni n. sp., Ammonellipsites menchikoffi n. sp., Ammonellipsites conradae n. sp., Muensteroceras fabrei n. sp., Eurites commutatus n. sp., Eurites pondus n. sp., subfamily Trimorphoceratinae n. subfam., Trimorphoceras n. gen., Trimorphoceras crassilens n. sp., Trimorphoceras absolutum n. sp., Trimorphoceras molestum n. sp., Obturgites n. gen., Obturgites polysarcus n. sp., Obturgites oligosarcus n. sp., Dzhaprakoceras dzhazairense n. sp., and Merocanites merocanites $\mathrm{n}$. sp. These species occur in one very prolific horizon and can be attributed to the North African Ammonellipsites-Merocanites Assemblage (Fascipericyclus-Ammonellipsites Genus Zone; Late Tournaisian to Early Viséan). They represent one of the most diverse ammonoid faunas known from this time interval.
\end{abstract}

\section{Introduction}

Ammonoids from the Tournaisian-Viséan boundary interval belong to the first ever described Palaeozoic cephalopods (Sowerby 1812-1814; Parkinson 1822). They were already monographically described in the 19th century; M'Coy (1844) and M'Coy \& Griffith (1862) described some species from Ireland, de Koninck (1880) was the pioneer in describing time equivalent faunas from Belgium, and Holzapfel (1889) gave an overview on the ammonoids from the Erdbach Limestone of the Rhenish Mountains in Germany. At the beginning of the 20th century, it was particularly Foord (1901, 1903) who revised the Irish Carboniferous ammonoids and who produced for that time the most extensive monographic description of the species around the Tournaisian-Viséan boundary.

Latest Tournaisian and earliest Viséan ammonoids were reported from a variety of geographic areas (Korn \& Ilg 2009):

Alaska - Gordon (1957) reported species of the genera Polaricyclus and Eurites from the Brooks Range; these genera occur in the Pericyclus-Progoniatites
Genus Zone and also in the Fascipericyclus-Ammonellipsites Genus Zone. Therefore it is not clear, which horizon is represented by this assemblage.

British Columbia - The Lower Mount Head Formation yielded a rather diverse fauna with the genera Merocanites, Imitoceras, Irinoceras, Goniocycloides, Furnishoceras, Eurites, and Dzhaprakoceras (Work \& Nassichuk 2000). An assemblage with such a composition is not known from other places, and hence, its stratigraphic correlation is difficult. It might be a time equivalent of the Dalle á Merocanites.

Utah - The assemblage from the Deseret Limestone (Petersen et al. 2000) is composed of only two species of Dzhaprakoceras; this makes it difficult to place it within the stratigraphic scheme.

Kentucky - Early, middle, and late Osagean ammonoid assemblages (Work \& Mason 2003, 2004, 2005) show a succession similar to the occurrences in Algeria. Particularly the fauna from the New Providence Shale (late Osagean) with Polaricyclus, Winchelloceras, and Michiganites (Work \& Mason 2004) may represent a time equivalent of the assemblage from the Dalle á Merocanites.

\footnotetext{
* Corresponding author
} 
Ireland - Crick (1899) and particularly Foord (1901, 1903) described a rich ammonoid fauna on the basis of well-preserved material. Their material came from a number of localities and from lithologically different sources, and because of this, both authors were not able to present a stratigraphic succession of the assemblages. The described species are probably belonging to the following genera: Dzhaprakoceras, Muensteroceras, Ammonellipsites, Irinoceras, Fascipericyclus, Rotopericyclus, Eurites, Asiacyclus (?), Neopericyclus (?), Merocanites, and Michiganites. Smyth (1951) described a fauna from the Rush Slates with Ammonellipsites, Dzhaprakoceras (?), Eurites, and Merocanites; it has a position close to the Tournaisian-Viséan boundary.

Lancashire - An assemblage with Ammonellipsites, Helicocyclus, Rotopericyclus, Dzhaprakoceras, Eonomismoceras, and Michiganites from the Leagram Mudstone Member has a position in the basalmost Viséan (Riley 1996).

Derbyshire and Staffordshire - Assemblages from the Peak district probably overspan the Tournaisian-Viséan boundary, with a lower (Tournaisian) fauna containing Fascipericyclus and a higher fauna with Ammonellipsites (Hind 1910; Tilsley \& Korn 2009).

Belgium - The classical assemblages first described by de Koninck $(1844,1880)$ and later revised by Delépine (1940) from the "Calcaire Waulsortien" are composed of the genera Muensteroceras, Dzhaprakoceras, Eurites, Fascipericyclus, and Hammatocyclus; they have probably a latest Tournaisian age.

Rhenish Mountains - The Erdbach Limestone, which occurs in pockets in the Devonian reef limestones, yielded species of the genera Muensteroceras, Dzhaprakoceras, Fascipericyclus (?), Ammonellipsites, Irinoceras, Merocanites, and possibly Dimorphoceras. The fauna was first described by Holzapfel (1889) and partly revised by Schmidt (1925). Conodont samples from the ammonoid-bearing rocks yielded a fauna that can be attributed to the latest Tournaisian (Krebs 1968).

Harz - Early Carboniferous limestones resting in cavities in the Devonian reef limestones yielded a rather rich latest Tournaisian assemblage (Schindewolf 1951), but it is not clear by how much it has been contaminated by younger (i.e. late Viséan) elements. It can be stated with some certainty that the genera Ammonellipsites, Irinoceras, Merocanites, Dzhaprakoceras, Eurites, Hammatocyclus, and Helicocyclus come from one single time interval. Possibly, Hammatocyclus was already described by Roemer (1852).

Cantabrian Mountains - The Alba Formation ("Marbre Griotte"; Kullmann 1961, 1963) of the Oviedo and León districts yielded a fauna with species of Dzhaprakoceras (very common), Eurites, Merocanites, and Michiganites, and very rarely Ammonellipsites, this formation can be correlated with the Dalle à Merocanites. Barrois (1879, 1882), and Delépine
(1943) already previously described a few elements of the fauna. Possibly, the Villabellaco Formation of the Palencia district (Higgins et al. 1964; Higgins \& Wagner-Gentis 1982) with the genera above and also Winchelloceras (?) and Pseudogirtyoceras has a similar age.

Sierra Morena in Central Iberia - The record of $\mathrm{Am}$ monellipsites places the Casa de la Varga Formation near the Tournaisian-Viséan boundary (Pardo Alonso \& Kullmann 2002).

Western Pyrenees - A fauna with Ammonellipsites, Dzhaprakoceras and Merocanites from the Aspe Valley is probably a time equivalent of the Iberian occurrences (Schmidt 1931).

Montagne Noire - The Calcaire de Faugères (Faugères Formation) yielded a small fauna with Ammonellipsites, Winchelloceras (?), and Merocanites; this diminished fauna makes it hard to state if they are representative of the latest Tournaisian or earliest Viséan age (Böhm 1935; Korn \& Feist 2007).

Carnic Alps - The published record of a Merocanites sp. from the Cellon (Müller 1959) is complimented by new and so far undescribed faunas containing Ammonellipsites, Dzhaprakoceras, and Irinoceras.

Holy Cross Mountains - The genera Ammonellipsites, Muensteroceras, Irinoceras, and Merocanites from the Radlin Formation at Galezice resemble the assemblages from other Central European occurrences (Czarniecki 1973; Dzik 1997).

North Urals and Pay Khoy - Kusina (1971, 1973, 1974, 1980, 1983, 2000), Shimansky \& Kusina (1977), and Kusina \& Konovalova (2004) described diverse faunas from localities at the Kozhim River, making it one of the key regions for understanding the ammonoid succession of the time interval around the Tournaisian-Viséan boundary. The succession possibly overspans the Tournaisian-Viséan boundary; it is dominated by the genera Muensteroceras and Dzhaprakoceras. The rich faunas show some peculiarities in their composition, with a number of genera not recorded from other places.

Iran - Dzhaprakoceras and Merocanites fossils may suggest the presence of a Tournaisian-Viséan boundary fauna in Central Iran (Walliser 1966).

Tien Shan of Kyrgyzstan - The area of the Dzhapryk River is the most important Asian occurrence of Late Tournaisian and Early Viséan ammonoids. Several horizons yielded rich faunas, which have a composition consisting of Fascipericyclus, Ammonellipsites, Dzhaprakoceras, Merocanites, etc. similar to those of the European occurrences (Librovitch 1927; Popov 1965, 1968, 1975).

Xinjiang - Muensteroceras, Dzhaprakoceras, and Merocanites are reported from the upper Donggulubastao Formation of the eastern Junggar Basin (Sheng 1984; Liang \& Wang 1988, 1991); it is difficult to tell the precise horizon of this occurrence.

New South Wales - Irinoceras and Merocanites from the Flagstaff Sandstone (Campbell et al. 1983) may 
have a stratigraphic position near the TournaisianViséan boundary.

This list shows that most of the described assemblages are very similar in their composition of genera and that a very distinctive provincialism of faunas obviously does not exist. The list also shows that the rich occurrences are geographically restricted to the European-North African-Central Asian realm. Indeed, time equivalent assemblages from other regions are poor in species, and they are mostly composed of the widespread genera Dzhaprakoceras and Merocanites.

Ammonoids from near the Tournaisian-Viséan boundary of the northern and Central Sahara were described several times:

Anti-Atlas of Morocco - The assemblage from the locality at the Bordj d'Erfoud possibly yielded the genera Muensteroceras, Dzhaprakoceras, Eurites, Ammonellipsites, Fascipericyclus, Rotopericyclus, Winchelloceras, and Merocanites as described by Delépine (1941); it was never re-investigated and requires revision. The rich fauna from Bouhamed (Korn et al. 2003a) is slightly older.

Saoura Valley of north-western Algeria - The fauna from the Hassi Sguilma Formation was the richest known African ammonoid assemblage of its age. Menchikoff (1930) and Pareyn (1961) described species possibly belonging to the genera Imitoceras, Irinoceras, Muensteroceras, Dzhaprakoceras, Eurites, Ammonellipsites, Neopericyclus, Fascipericyclus, Rotopericyclus, Winchelloceras (?), and Merocanites, i.e. a composition similar to the occurrence of the Bordj d'Erfoud. Pareyn's descriptions are partly based on poorly preserved material difficult to interpret. Re-sampling of the locality at Hassi Sguilma by the present authors (Korn et al. 2010b) revealed that the composition of the fauna shows some differences to the fauna from the Dalle à Merocanites. Further studies are required to solve this problem.

Gourara of western Algeria - The specimens from the Dalle à Merocanites figured by Conrad (1984) belong to the genera Ammonellipsites and Merocanites; they come from the same locality, which will be outlined in greater detail here.

Ahnet and Mouydir of South Algeria - Conrad (1984, p. 151) listed a number of ammonoid species from the Dalle des Iridet but did not Figure specimens. New findings from these regions will be described in a separate article (Korn et al. 2010b).

It was particularly the third occurrence, located $14.5 \mathrm{~km}$ west-southwest of Timimoun, which turned out to be very productive for ammonoid faunas; it yielded a large suite of material during the field trips by the present authors. This fauna will be described here for the first time to compliment the monographs of the Early Tournaisian (Ebbighausen et al. 2004), early Late Tournaisian (Korn et al. 2010c, 2010a), as well as Early and Middle Viséan (Bockwinkel et al. 2010) assemblages from the Central Sahara of Algeria.

\section{Geological setting}

The ammonoid fauna described here comes from a locality $14.5 \mathrm{~km}$ west-southwest of Timimoun (Gourara, western Algeria), at the south-western margin of the Grand Erg Occidental (Fig. 1). Between the sand dunes of the Grand Erg, Carboniferous sedimentary rocks are exposed, extending over a distance of approximately $40 \mathrm{~km}$ in a SW-NE erosion window that ranges in width between a few hundreds of metres and $10 \mathrm{~km}$.
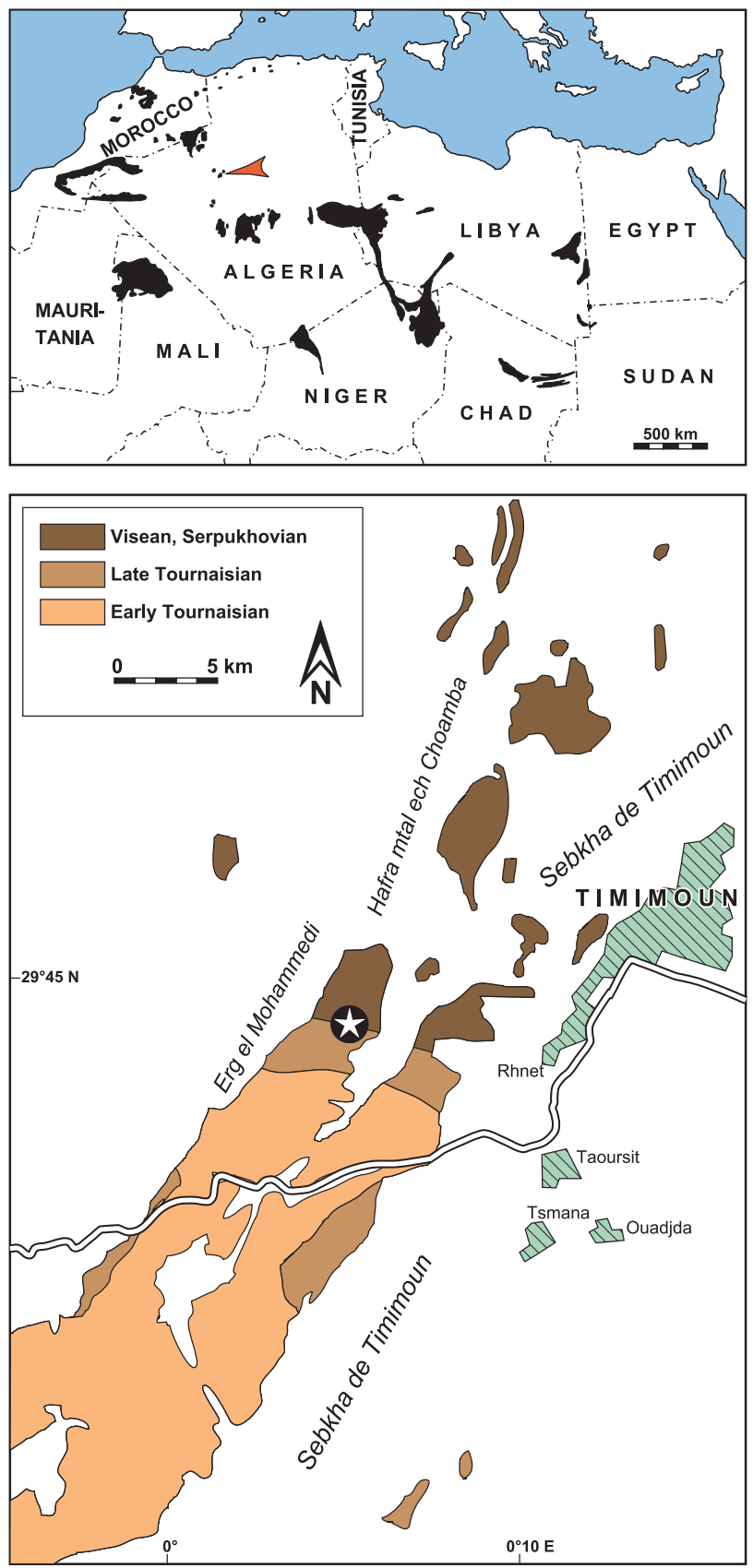

Figure 1. The outcrop of Palaeozoic rocks in northern Africa and Iberia and the geographic position (marked by an asterisk) of the Dalle à Merocanites near Timimoun. 

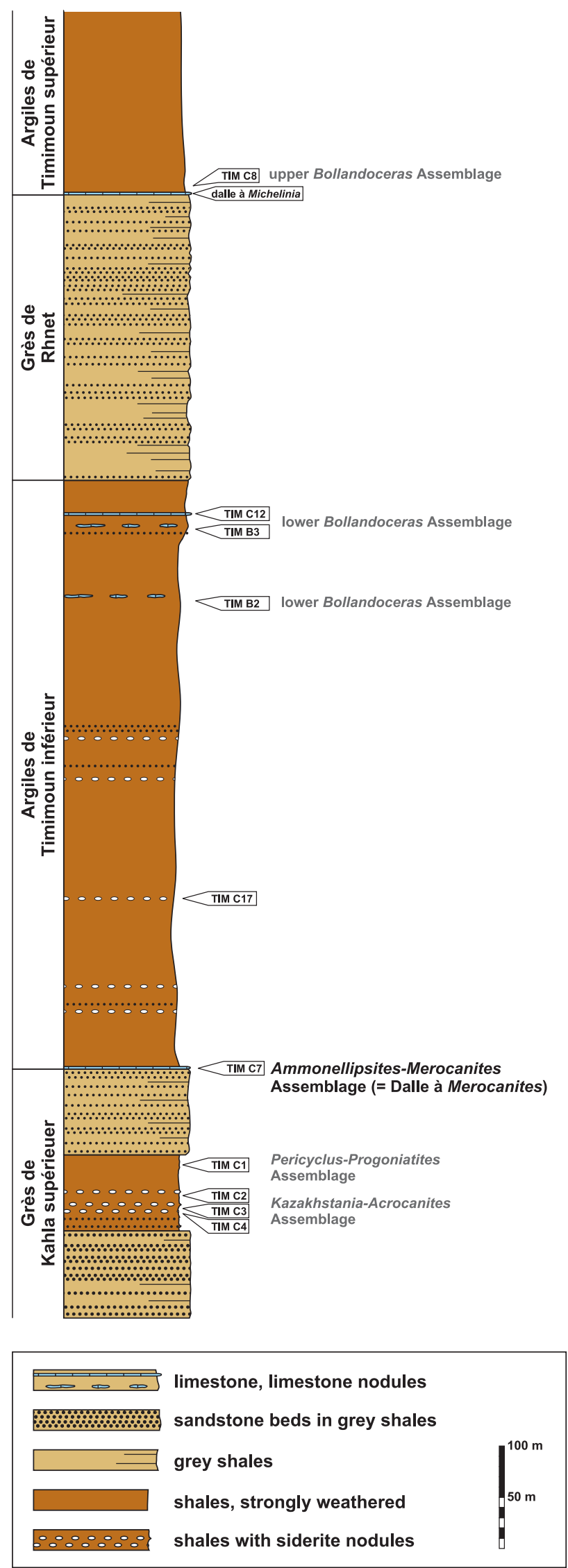

Figure 2. Stratigraphic section of the Early Carboniferous (Mississippian) rock succession near Timimoun with the position of the fauna from the Dalle à Merocanites.
The rocks are gently inclined towards the north-east. Its succession (Conrad 1984) consists mainly of shales with several intercalated sandstone formations; carbonates are very rare and restricted to distinct horizons.

The stratigraphically oldest of the carbonate horizons within the Carboniferous succession is the Dalle à Merocanites, a unit of approximately $1.90 \mathrm{~m}$ thickness, composed of a few mainly sideritic and dolomitized nodular beds separated by strongly weathered shales (Fig. 2). The Dalle à Merocanites rests immediately on top of the highest sandstone complex of the Upper Kahla Shales, which is about $100 \mathrm{~m}$ thick. It comprises a rock unit composed of silty shales with a large number of thin rhythmic sandstone beds. Both units form a low ridge that forms an outcrop that can be studied over a lateral distance of about $2.5 \mathrm{~km}$. The Dalle à Merocanites is located at the northern flank of this ridge. It is overlain by the Lower Timimoun Shales (Argiles de Timimoun infèrieur), a very strongly weathered shale unit that forms a wide sebkha without any outcrop. All ammonoids described here come from a single horizon at the base of the Dalle à Merocanites.

\section{Biostratigraphy and palaeogeography}

The newly defined Tournaisian-Viséan boundary (Devuyst et al. 2003) is, in most ammonoid-bearing sections, characterised by the genera Ammonellipsites and Merocanites (Riley 1990; Korn et al. 2007; Tilsley \& Korn 2009). Both genera have been recorded from both sides of the boundary. Konovalova (2005) gave an overview on the ammonoid diversity patterns across the Tournaisian-Viséan boundary.

Occurrences in Germany at Erdbach (Rhenish Mountains) and Iberg (Harz Mountains), where the two genera co-occur with species of Dzhaprakoceras, Eurites and others, have been investigated in terms of conodont stratigraphy. Both belong in the anchoralis Zone (Krebs 1968; Gischler 1992), which speaks for a correlation with the latest Tournaisian. By contrast, the occurrence of Ammonellipsites in the Leagram Mudstone of Clitheroe (Lancashire) has to be placed in the lowest Viséan (Riley 1996). This age determination is based on foraminifers.

Based on the occurrence of Ammonellipsites and Merocanites alone, it cannot be decided if the Dalle à Merocanites has a Tournaisian or a Viséan age (Fig. 3). The few other faunal elements provide little evidence for a precise stratigraphic determination. Hammatocyclus is known from the Harz Mountains (i.e. with a Tournaisian age); the occurrence of Dzhaprakoceras is often an indication for the earliest Viséan (Popov 1968; Riley 1996).

The slightly imprecise stratigraphic assignment of the assemblage from the Dalle à Merocanites and other occurrences is an obstacle for a quantitative palaeogeographic analysis of the various North African occurrences (Wendt et al. 2009; Korn et al. 2010b). Differences in the composition of the assemblages may be 


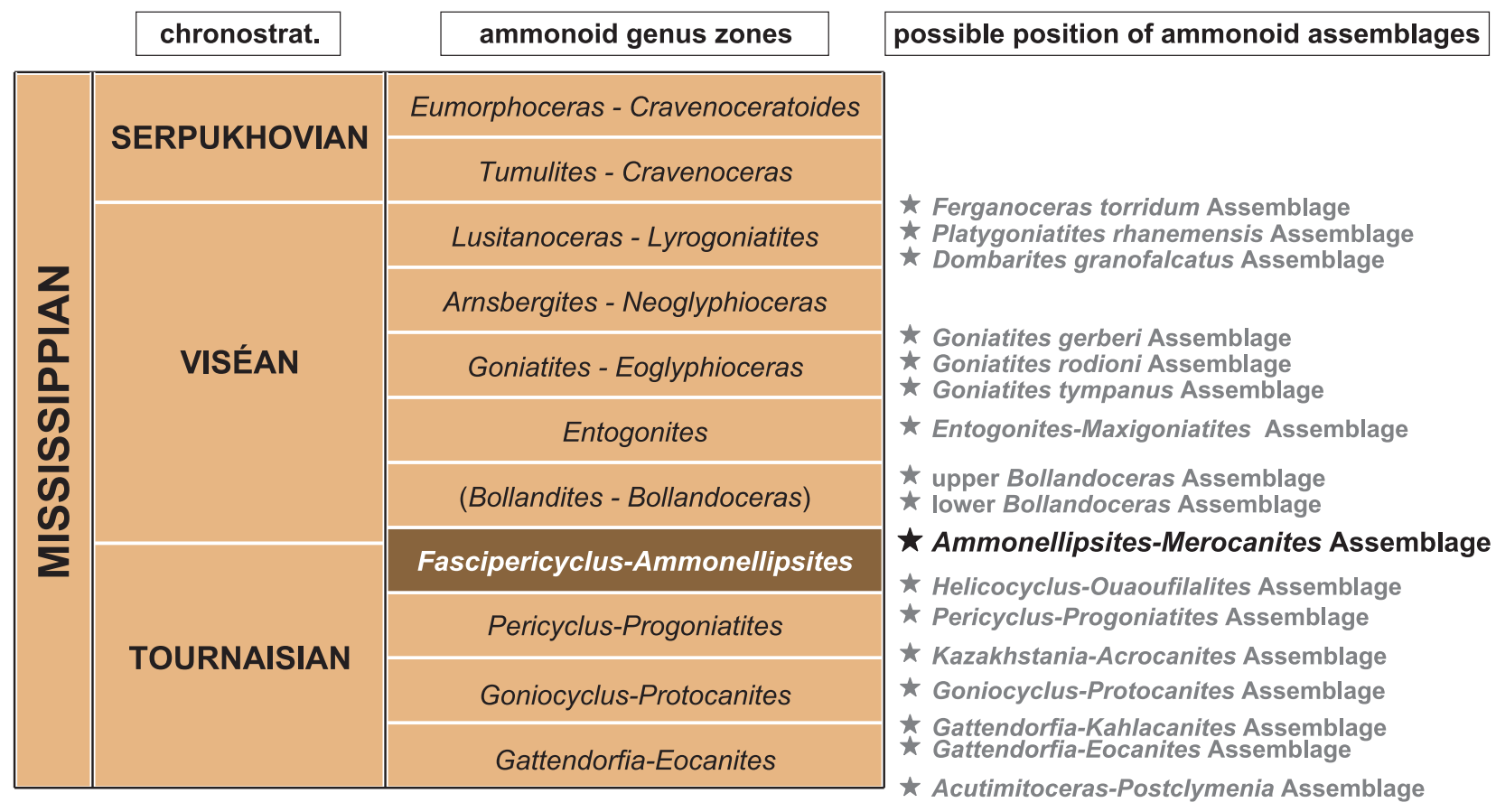

Figure 3. Stratigraphic scheme of the Early Carboniferous (Mississippian) chronostratigraphy and ammonoid zonation, with correlation of the North African ammonoid assemblages (after Korn et al. 2004, 2007). The position of the fauna from the Dalle à Merocanites is highlighted.

caused by the different age of the faunas. It can be stated, however, that the interval around the Tournaisian-Viséan boundary is, in contrast to the Late Viséan (Korn 1997; Korn et al. 2005; Korn \& Ebbighausen 2008), a time of ammonoid cosmopolitanism. The wide geographic range of many of the genera, which compose similar communities is suggestive of such a situation. Ammonellipsites, for instance, has a geographic range in the equatorial zone between north-western Africa and Central Asia, and Dzhaprakoceras as well as Merocanites have an almost global distribution. It has to be kept in mind, however, that many occurrences require revision for a detailed analysis. Furthermore, the lack of taxa (e.g. the Goniatitida; see Korn et al. 2005), which are known from below and above the interval studied here, indicate that the knowledge of the faunas is still limited.

\section{Material}

At total of more than 800 specimens were investigated from the Dalle à Merocanites in sample TIM-C7, $14.5 \mathrm{~km}$ west-southwest of Timimoun $\left(29.2275^{\circ} \mathrm{N}, 0.0855^{\circ} \mathrm{E}\right)$; approximately 640 specimens can be assigned to the following species:

\author{
Hammatocyclus pollex $\mathrm{n}$. sp. \\ Hammatocyclus corrugatus n. $\mathrm{sp}$. \\ Neopericyclus arenosus n. sp. \\ Ammonellipsites pareyni $\mathrm{n}$. sp. \\ Ammonellipsites menchikoffi $\mathrm{n}$. sp. \\ Ammonellipsites conradae n. sp. \\ Muensteroceras fabrei $\mathrm{n}$. $\mathrm{sp}$. \\ Eurites commutatus n. sp. \\ Eurites pondus n. sp. \\ Trimorphoceras crassilens $\mathrm{n}$. $\mathrm{sp}$. \\ Trimorphoceras absolutum n. sp. \\ Trimorphoceras molestum n. sp. \\ Obturgites polysarcus n. sp. \\ Obturgites oligosarcus n. sp. \\ Dzhaprakoceras dzhazairense $\mathrm{n}$. sp. \\ Merocanites merocanites $\mathrm{n}$. $\mathrm{sp}$.
}

\section{Systematic descriptions}

The descriptive part of this monograph will mainly focus on the illustration and morphometric analysis of the species with particular attention to their ontogenetic devel-

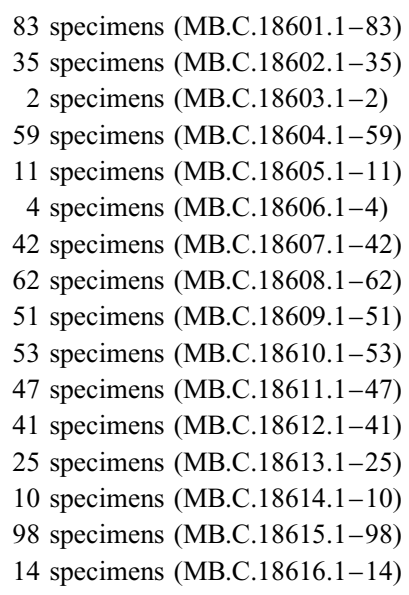

opment. For this reason, more than 100 cross sections were produced (of which nearly 50 are illustrated here). The key for the description of the species, including explanation of methods, is published by Korn (2010). Sutural terminology follows Korn et al. (2003b). 
Order Goniatitida Hyatt, 1884

Suborder Goniatitina Hyatt, 1884

Superfamily Pericyclaceae Hyatt, 1900

Family Pericyclidae Hyatt, 1900

\section{Subfamily Hammatocyclinae n. subfam.}

Subfamily definition. Pericyclidae with significant ontogenetic changes of conch geometry; juveniles thickly discoidal and evolute with broad, slightly flattened venter; adult stage pachyconic, subinvolute to subevolute with increasing height of the aperture; umbilical wall often flattened, umbilical margin narrowly rounded or subangular. Ornament with almost linear rectiradiate growth lines; growth lines sometimes strengthened to fine riblets; shell constrictions extend parallel to the growth lines. Suture line with very narrow, parallel-sided or slightly pouched external lobe with straight or weakly sinuous flanks and low median saddle; ventrolateral saddle narrowly rounded or subangular, adventive lobe lanceolate.

Included genera.

Hammatocyclus Schindewolf, 1951

Neopericyclus Popov, 1965

\section{Hammatocyclus Schindewolf, 1951}

Type species. Pericyclus (Hammatocyclus) homoceratoides Schindewolf, 1951 (OD).

Genus definition. Pericyclinae with significant ontogenetic changes of conch geometry; juveniles thickly discoidal and evolute with broad, slightly flattened venter; adult stage pachyconic, subinvolute to subevolute (Fig. 4) with increasing height of the aperture; umbilical wall often flattened, umbilical margin narrowly rounded or subangular. Ornament with very fine and almost linear rectiradiate growth lines, sometimes forming a very wide and shallow ventral sinus; growth lines sometimes strengthened to fine riblets; shell constrictions extend parallel to the growth lines. Suture line with very narrow, parallel-sided or slightly pouched external lobe with straight or weakly sinuous flanks and low median saddle; ventrolateral saddle narrowly rounded or subangular, adventive lobe lanceolate.

Included species.

confinis: Hammatocyclus confinis Kusina in Shimansky \& Kusina, 1977, p. 89; North Urals.

corrugatus: Hammatocyclus corrugatus n. sp.; Gourara, Algeria

homoceratoides: Pericyclus (Hammatocyclus) homoceratoides Schindewolf, 1951, p. 82; Harz.

incertus: Hammatocyclus incertus Kusina \& Konovalova, 2004, p. 18; North Urals.

latumbilicatus: Muensteroceras latumbilicatum Kullmann, 1961, p. 266; Cantabrian Mountains.

pollex: Hammatocyclus pollex n. sp.; Gourara, Algeria.

? brazerensis: Pericyclus (Hammatocyclus) brazerensis Gordon, 1986, p. 31; Utah.

? crenulatus: Goniatites crenulatus De Koninck, 1880, p. 112; Belgium.

? tumidus: Goniatites tumidus Roemer, 1852, p. 94; Harz.

? tuberculatus: "Goniatites" (Pericyclus?) tuberculatus Delépine, 1940, p. 42; Belgium.

Separation of the new species. The two species of Hammatocyclus from the Dalle à Merocanites can be separated by their conch morphology and ornament. In the conch shape and its ontogenetic development, they are characterised as follows:

- Hammatocyclus pollex - conch thinly pachyconic in the adult stage (ww/dm $=0.60-0.75$ at $60 \mathrm{~mm} \mathrm{dm}$ ); ww/wh ratio $1 \mathrm{ess}$ than 2.00 above $40 \mathrm{~mm} \mathrm{dm}$; uw/dm ratio less than 0.30 above $60 \mathrm{~mm} \mathrm{dm}$;

- Hammatocyclus corrugatus - conch thickly pachyconic in the adult stage (ww/dm $=0.75-0.85$ at $60 \mathrm{~mm} \mathrm{dm}$ ); ww/wh ratio usually more than 2.00 above $40 \mathrm{~mm} \mathrm{dm}$; uw/dm ratio usually more than 0.30 above $60 \mathrm{~mm} \mathrm{dm}$.
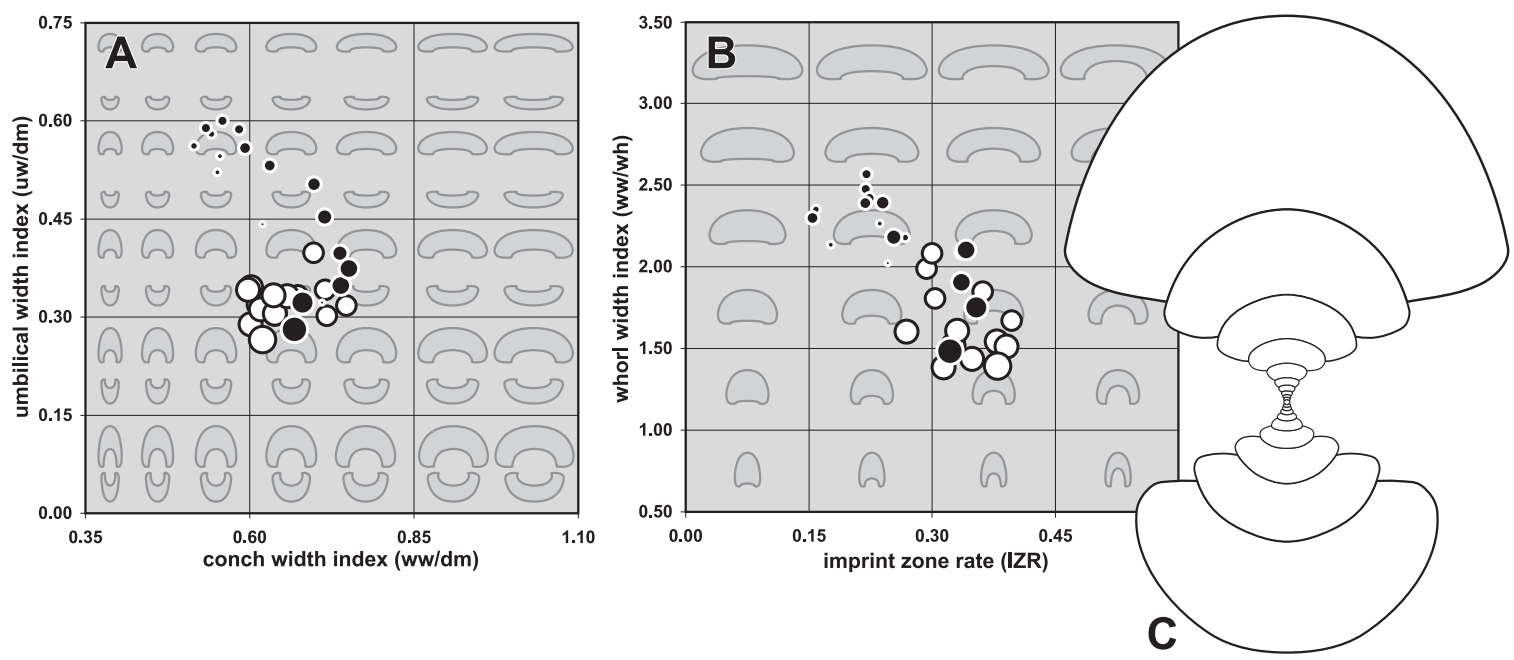

Figure 4. Ontogenetic trajectories of Hammatocyclus, exemplified for Hammatocyclus pollex n. sp. from locality TIM-C7. A. Ontogenetic development of the conch width index (ww/dm), umbilical width index (uw/dm). B. Ontogenetic development of the imprint zone rate (IZR), whorl width index (ww/wh). C. Cross section of paratype MB.C.18601.7; $\times 1.0$. [Black dots represent ontogenetic stages of cross section MB.C.18601.7, white dots represent the reference specimens (Tab. 2).] 
The ornament differs as follows:

- Hammatocyclus pollex - without riblets;

- Hammatocyclus corrugatus - riblets extending on the venter, causing a corrugated surface.

Discussion. Hammatocyclus differs from other genera, which may show a rather similar adult morphology, in the presence of an evolute juvenile stage. The most similar genus is probably Rotopericyclus Turner, 1948, in which some species possess a subevolute adult stage, but in this the juvenile stage is either subevolute or subinvolute. Parahammatocyclus Riley, 1996 possesses a similar conch morphology, but differs in the V-shaped external lobe.

\section{Hammatocyclus pollex n. sp.}

Figures 5-7

Derivation of name. After Lat. pollex = thumb, because of the thumb-shaped outline of the ventrolateral saddle.

Holotype. Specimen MB.C.18601.1, illustrated in Figure 5.

Type locality and horizon. Sebkha de Timimoun, locality TIM-C7 (14.5 km west-southwest of Timimoun, Algeria); Ammonellipsites-Merocanites Assemblage.

Material. Very well preserved material is available. In total, 83 specimens between 17 and 80 mm conch diameter were studied. Holotype MB.C.18601.1 is, at $80 \mathrm{~mm}$ conch diameter, still fully chambered; this suggests that the total diameter of the specimen was at least $150 \mathrm{~mm}$. Most of the specimens are internal moulds of the phragmocone with minor parts of the body chamber preserved, and many show somewhat corroded shell remains.

Diagnosis. Hammatocyclus with a thickly discoidal and evolute conch at $2-6 \mathrm{~mm} \mathrm{dm}$; conch thinly pachyconic between 10 and $80 \mathrm{~mm}$ dm with an almost continuous trend from evolute to subinvolute; whorl cross section strongly depressed in juveniles and moderately depressed in adults; umbilical margin rounded in juveniles, becoming subangular at $10 \mathrm{~mm} \mathrm{dm}$ and angular at $30 \mathrm{~mm} \mathrm{dm}$; umbilical wall flat and slightly oblique in late growth stages; aperture very low in juveniles and continuously becoming moderately high to high during ontogeny. Ornament with very fine and almost linear rectiradiate growth lines; external sinus very shallow; growth lines strengthened to form fine riblets particularly on the flanks; shell constrictions parallel to the growth lines up to $20-25 \mathrm{~mm} \mathrm{dm}$, large stage without constrictions; very weak umbilical rim, sometimes with delicate nodes formed by the shell. Suture line with very narrow, parallel-sided and slightly pouched external lobe and low median saddle; ventrolateral saddle narrowly rounded and sometimes slightly inflated, adventive lobe lanceolate, asymmetric.

Table 1. Conch ontogeny (Figs 7A-G, L-N) of Hammatocyclus pollex n. sp.

\begin{tabular}{|c|c|c|c|}
\hline $\mathrm{dm}$ & conch shape & whorl cross section shape & aperture \\
\hline $4 \mathrm{~mm}$ & $\begin{array}{l}\text { thickly discoidal; evolute } \\
(\mathrm{ww} / \mathrm{dm}=0.52-0.58 ; \mathrm{uw} / \mathrm{dm}=0.50-0.60)\end{array}$ & $\begin{array}{l}\text { strongly depressed; moderately embracing } \\
(\mathrm{ww} / \mathrm{wh}=2.00-2.40 ; \mathrm{IZR}=0.15-0.25)\end{array}$ & $\begin{array}{l}\text { very low to low } \\
(\text { WER }=1.45-1.55)\end{array}$ \\
\hline $10 \mathrm{~mm}$ & $\begin{array}{l}\text { thinly pachyconic; evolute } \\
(\mathrm{ww} / \mathrm{dm}=0.62-0.70 ; \mathrm{uw} / \mathrm{dm}=0.50-0.56)\end{array}$ & $\begin{array}{l}\text { strongly to very strongly depressed; moderately } \\
\text { embracing ( } w w / w h=2.30-2.70 ; I Z R=0.20-0.25 \text { ) }\end{array}$ & $\begin{array}{l}\text { low } \\
(\text { WER }=1.50-1.65)\end{array}$ \\
\hline $30 \mathrm{~mm}$ & $\begin{array}{l}\text { thinly to thickly pachyconic; subevolute } \\
\text { (ww/dm }=0.65-0.80 ; \mathrm{uw} / \mathrm{dm}=0.30-0.40 \text { ) }\end{array}$ & $\begin{array}{l}\text { moderately to strongly depressed; strongly embracing } \\
\text { (ww/wh }=1.50-2.20 ; \text { IZR }=0.30-0.35 \text { ) }\end{array}$ & $\begin{array}{l}\text { low } \\
(\text { WER }=1.60-1.75)\end{array}$ \\
\hline $60 \mathrm{~mm}$ & $\begin{array}{l}\text { thinly pachyconic; subinvolute } \\
(\mathrm{ww} / \mathrm{dm}=0.60-0.75 ; \mathrm{uw} / \mathrm{dm}=0.25-0.30)\end{array}$ & $\begin{array}{l}\text { moderately depressed; strongly embracing } \\
(\mathrm{ww} / \mathrm{wh}=1.50-1.90 ; \mathrm{IZR}=0.30-0.35)\end{array}$ & $\begin{array}{l}\text { moderate to high } \\
\text { (WER }=1.90-2.05)\end{array}$ \\
\hline
\end{tabular}
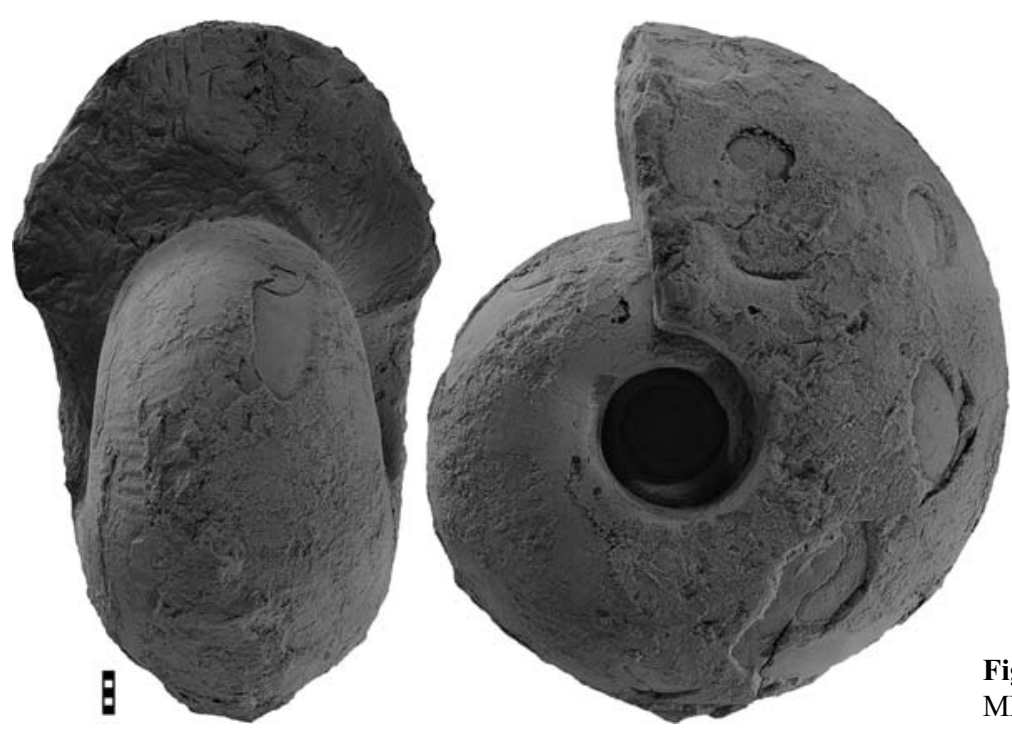

Figure 5. Hammatocyclus pollex n. sp., holotype MB.C.18601.1 from locality TIM-C7; ×1.0. 
Table 2. Conch dimensions (in $\mathrm{mm}$ ) and proportions for reference specimens of Hammatocyclus pollex $\mathrm{n}$. sp.

\begin{tabular}{lcccccccccc}
\hline & $\mathrm{dm}$ & ww & wh & uw & ah & ww/dm & ww/wh & uw/dm & WER & IZR \\
\hline holotype MB.C.18601.1 & 80.3 & 49.8 & 35.7 & 21.3 & 22.1 & 0.62 & 1.40 & 0.27 & 1.90 & 0.38 \\
paratype MB.C.18601.4 & 62.1 & 37.4 & 27.0 & 18.0 & 18.5 & 0.60 & 1.39 & 0.29 & 2.03 & 0.31 \\
paratype MB.C.18601.2 & 56.9 & 34.9 & 24.2 & 18.2 & 15.8 & 0.61 & 1.44 & 0.32 & 1.91 & 0.35 \\
paratype MB.C.18601.3 & 53.2 & 34.0 & 22.3 & 16.3 & 15.1 & 0.64 & 1.52 & 0.31 & 1.94 & 0.33 \\
paratype MB.C.18601.23 & 50.5 & 31.1 & 20.3 & 15.7 & 12.6 & 0.62 & 1.53 & 0.31 & 1.77 & 0.38 \\
paratype MB.C.18601.13 & 49.1 & 29.4 & 20.7 & 16.0 & 13.0 & 0.64 & 1.52 & 0.31 & 1.94 & 0.33 \\
paratype MB.C.18601.5 & 43.6 & 28.7 & 17.8 & 14.5 & 11.9 & 0.66 & 1.61 & 0.33 & 1.89 & 0.33 \\
paratype MB.C.18601.16 & 42.8 & 25.8 & 16.0 & 14.8 & 11.7 & 0.60 & 1.61 & 0.35 & 1.90 & 0.27 \\
paratype MB.C.18601.21 & 42.3 & 26.9 & 17.4 & 14.1 & 10.8 & 0.64 & 1.55 & 0.33 & 1.80 & 0.38 \\
paratype MB.C.18601.17 & 40.8 & 24.4 & 16.1 & 13.9 & 9.8 & 0.60 & 1.51 & 0.34 & 1.73 & 0.39 \\
paratype MB.C.18601.19 & 37.0 & 26.5 & 15.8 & 11.2 & 9.6 & 0.72 & 1.67 & 0.30 & 1.82 & 0.40 \\
paratype MB.C.18601.20 & 36.3 & 25.9 & 14.3 & 12.4 & 10.0 & 0.71 & 1.81 & 0.34 & 1.90 & 0.30 \\
paratype MB.C.18601.6 & 35.3 & 26.4 & 13.2 & 11.2 & 9.4 & 0.75 & 1.99 & 0.32 & 1.85 & 0.29 \\
paratype MB.C.18601.18 & 34.8 & 23.5 & 12.7 & 11.6 & 8.1 & 0.67 & 1.85 & 0.33 & 1.70 & 0.36 \\
paratype MB.C.18601.22 & 28.7 & 20.0 & 9.6 & 11.5 & 6.7 & 0.70 & 2.09 & 0.40 & 1.70 & 0.30 \\
\hline
\end{tabular}
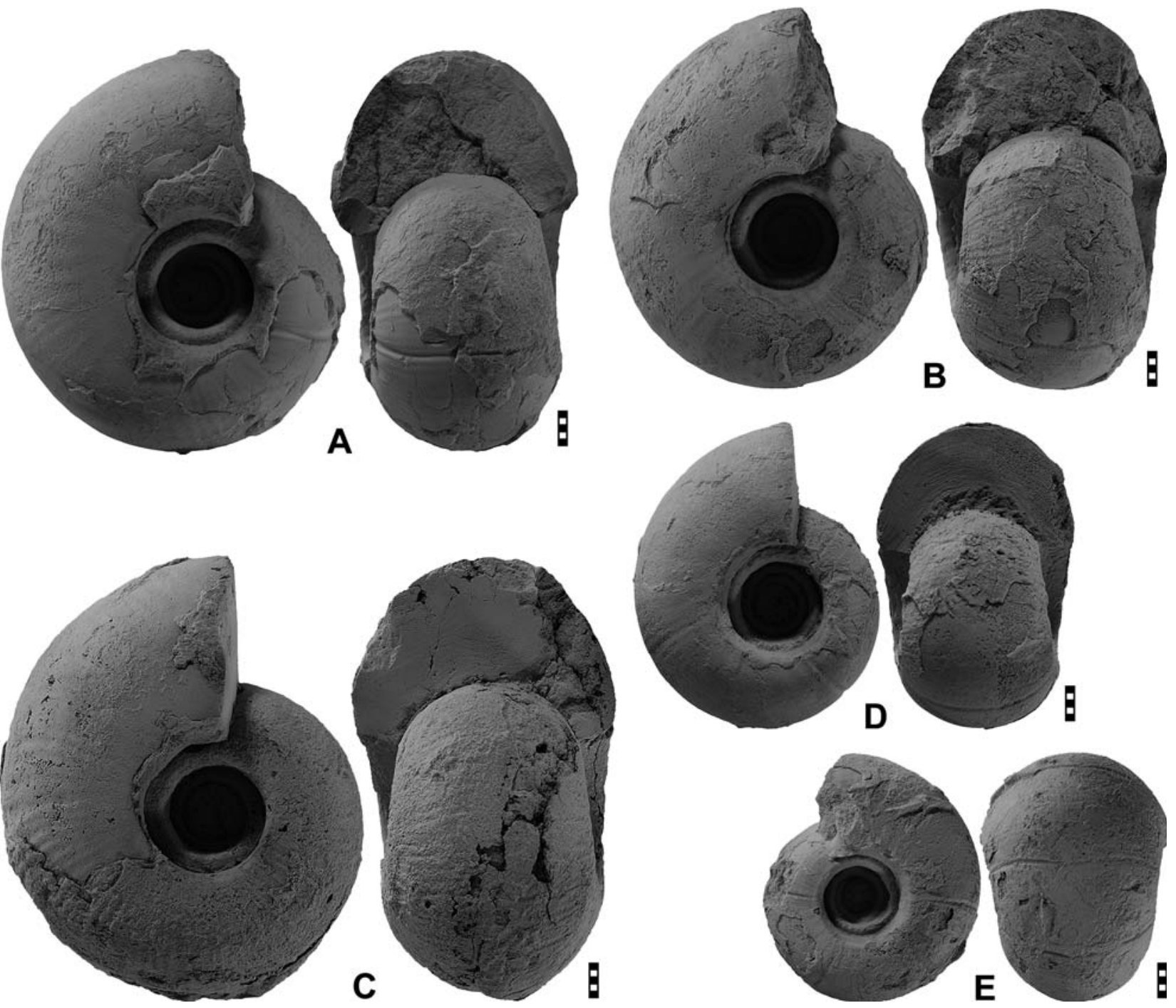

Figure 6. Hammatocyclus pollex n. sp. from locality TIM-C7; all $\times 1.0$. A. Paratype MB.C.18601.2. B. Paratype MB.C.18601.3. C. Paratype MB.C.18601.4. D. Paratype MB.C.18601.5. E. Paratype MB.C.18601.6. 


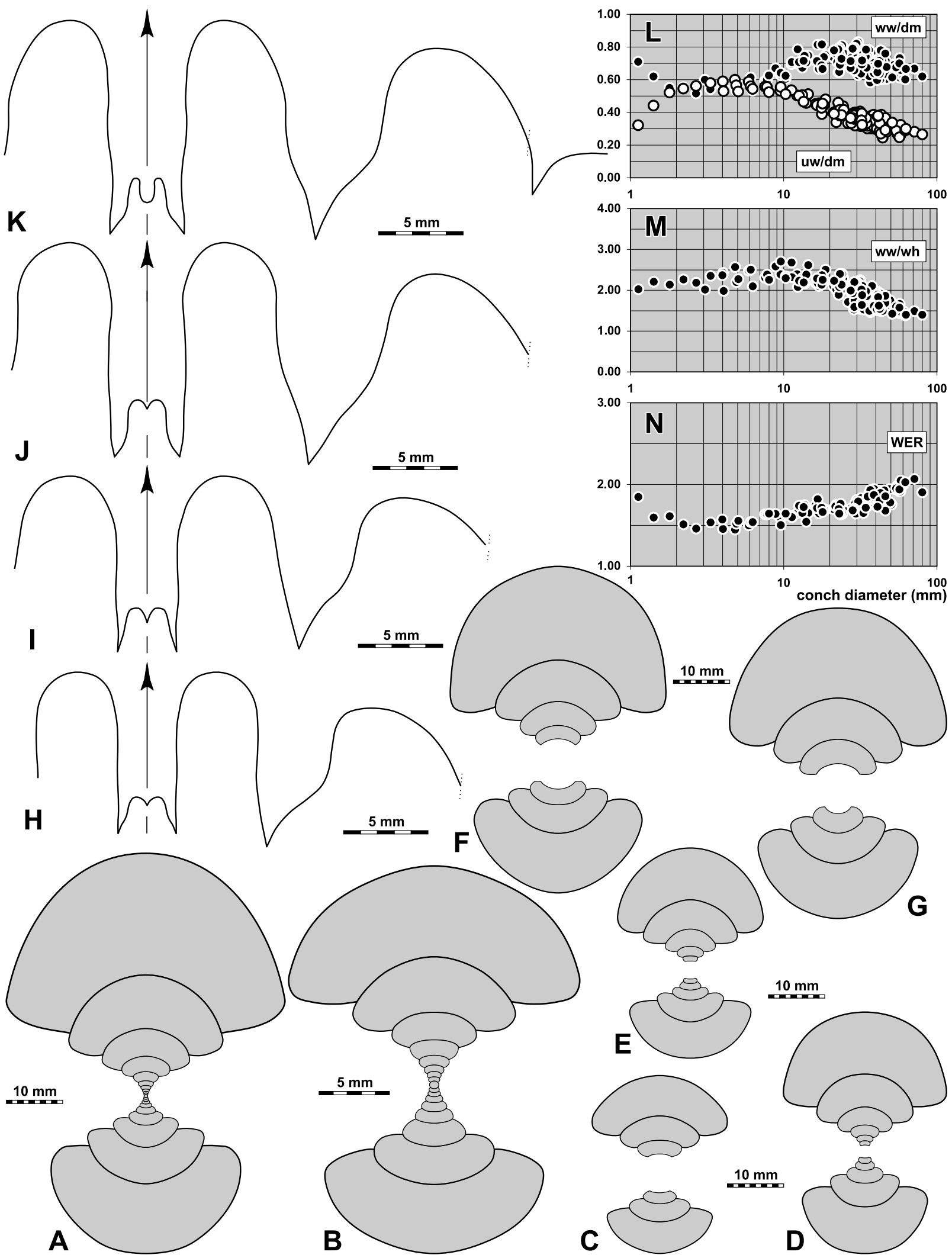

Figure 7. Hammatocyclus pollex n. sp. from locality TIM-C7. A. Cross section of paratype MB.C.18601.7; $\times 1.0$. B. Cross section (inner whorls) of the same specimen; $\times 2.5$. C. Cross section of paratype MB.C.18601.8; $\times 1.0$. D. Cross section of paratype MB.C.18601.9; $\times 1.0$. E. Cross section of paratype MB.C.18601.10; $\times 1.0$. F. Cross section of paratype MB.C.18601.11; × 1.0. G. Cross section of paratype MB.C.18601.12; ×1.0. H. Suture line of paratype MB.C.18601.13, at $21.6 \mathrm{~mm} \mathrm{ww}, 13.0 \mathrm{~mm}$ wh; $\times$ 3.0. I. Suture line of paratype MB.C.18601.14, at $25.3 \mathrm{~mm} w w, 15.5 \mathrm{~mm} w \mathrm{~h} \times 3$ 3.0. J. Suture line of paratype MB.C.18601.2, at $42.2 \mathrm{~mm} \mathrm{dm}, 27.1 \mathrm{~mm}$ ww, $15.0 \mathrm{~mm}$ wh; $\times 3.0$. K. Suture line of paratype MB.C.18601.15, at $29.6 \mathrm{~mm}$ ww, $15.2 \mathrm{~mm}$ wh; $\times 3.0$. L-N. Ontogenetic development of the conch width index (ww/dm), umbilical width index (uw/dm), whorl width index (ww/wh), and whorl expansion rate (WER) of all available specimens. 
Table 3. Suture line proportions (Figs 7H-K) for specimens of Hammatocyclus pollex $\mathrm{n}$. sp.

\begin{tabular}{llllllll}
\hline specimen & at dm & EL w/d & EL/VLS & EL/AL & MS h & VLS w/h & remarks \\
\hline paratype MB.C.18601.15 & c. $48.0 \mathrm{~mm}$ & 0.37 & 0.79 & 0.82 & 0.26 & 0.47 & flanks of A lobe strongly curved \\
paratype MB.C.18601.2 & $42.2 \mathrm{~mm}$ & 0.35 & 0.82 & 0.74 & 0.27 & 0.43 & \\
paratype MB.C.18601.14 & c. $34.0 \mathrm{~mm}$ & 0.35 & 0.60 & 0.81 & 0.25 & 0.57 & A lobe asymmetric \\
paratype MB.C.18601.13 & c. $28.0 \mathrm{~mm}$ & 0.36 & 0.70 & 0.78 & 0.23 & 0.51 & E lobe parallel-sided \\
\hline
\end{tabular}

Discussion. The new species resembles the type species of the genus, Hammatocyclus homoceratoides (Schindewolf, 1951), but can be separated by the lack of distinct umbilical nodes and coarse riblets on the flanks, which both are present in the type species.

The co-occurring H. corrugatus differs in the wider conch, the wider umbilicus, and the stronger ribs from H. pollex. H. confinis Kusina, 1977 has a slightly more slender conch at $40 \mathrm{~mm} \mathrm{dm}(\mathrm{ww} / \mathrm{dm}=0.66)$ with a slightly narrower umbilicus $(\mathrm{uw} / \mathrm{dm}=0.31$ ); this species has a suture line with slightly incurved flanks of the external lobe (in contrast to the gently pouched $\mathrm{E}$ lobe in the new species).

H. incertus Kusina \& Konovalova, 2004 is known from two small specimens (less than $30 \mathrm{~mm} \mathrm{dm}$ ). The external lobe has incurved flanks in this stage and does not show specific characters, but the shell surface shows a distinct umbilical rim, which is not present in $H$. pollex at this diameter.

\section{Hammatocyclus corrugatus $\mathbf{n}$. sp.}

Figures 8, 9

Derivation of name. After Lat. corrugo = with wrinkles, because of the shell surface.

Holotype. Specimen MB.C.18602.1, illustrated in Figure 8C.

Type locality and horizon. Sebkha de Timimoun, locality TIM-C7 (14.5 km west-southwest of Timimoun, Algeria); Ammonellipsites-Merocanites Assemblage.

Material. A total of 35 specimens between 25 and $79 \mathrm{~mm}$ conch diameter are available for study. The largest specimen is a fragment of the phragmocone with $55 \mathrm{~mm}$ whorl width; this suggests a total diameter of $80 \mathrm{~mm}$ for the protoconch and a diameter of $150 \mathrm{~mm}$ with body chamber. Most of the specimens are somewhat corroded phragmocones with parts of the body chamber; shell remains exist on some specimens.

Diagnosis. Hammatocyclus with a thickly discoidal and evolute conch at 2-6 $\mathrm{mm} \mathrm{dm}$; conch thickly pachyconic between 10 and $60 \mathrm{~mm}$ dm with an almost continuous trend from evolute to subevolute; whorl cross section strongly or very strongly depressed throughout ontogeny; umbilical margin rounded in juveniles, becoming subangular at $10 \mathrm{~mm} \mathrm{dm}$ and angular later in ontogeny; umbilical wall flat and slightly oblique in late growth stages; aperture low in juveniles and continuously becoming moderately high during ontogeny. Ornament with fine and almost linear rectiradiate growth lines and shallow riblets; external sinus very shallow and very wide; growth lines strengthened to fine riblets on flanks and venter; shell constrictions parallel to the growth lines; very weak umbilical rim, sometimes with delicate nodes formed by the shell. Suture line with very narrow, parallel-sided external lobe and low median saddle; ventrolateral saddle rounded, adventive lobe lanceolate, asymmetric.

Table 4. Conch ontogeny (Figs 9A-C, G-I) of Hammatocyclus corrugatus n. sp.

\begin{tabular}{|c|c|c|c|}
\hline $\mathrm{dm}$ & conch shape & whorl cross section shape & aperture \\
\hline $4 \mathrm{~mm}$ & $\begin{array}{l}\text { thickly discoidal; evolute } \\
\text { (ww/dm } \sim 0.58 ; \text { uw/dm } \sim 0.55 \text { ) }\end{array}$ & $\begin{array}{l}\text { strongly depressed; moderately embracing } \\
\text { (ww/wh } \sim 2.40 ; \text { IZR } \sim 0.20 \text { ) }\end{array}$ & $\begin{array}{l}\text { low } \\
\text { (WER } \sim 1.60)\end{array}$ \\
\hline $10 \mathrm{~mm}$ & $\begin{array}{l}\text { thickly pachyconic; evolute } \\
\text { (ww/dm } \sim 0.75 ; \text { uw/dm } \sim 0.52 \text { ) }\end{array}$ & $\begin{array}{l}\text { very strongly depressed; moderately embracing } \\
\text { (ww/wh } \sim 2.55 ; \text { IZR } \sim 0.20 \text { ) }\end{array}$ & $\begin{array}{l}\text { low } \\
\text { (WER 1.70) }\end{array}$ \\
\hline $30 \mathrm{~mm}$ & $\begin{array}{l}\text { thickly pachyconic; subevolute } \\
(\mathrm{ww} / \mathrm{dm}=0.75-0.85 ; \mathrm{ww} / \mathrm{dm}=0.35-0.45)\end{array}$ & $\begin{array}{l}\text { strongly depressed; strongly embracing } \\
\text { (ww/wh }=2.10-2.50 ; \text { IZR }=0.30-0.35 \text { ) }\end{array}$ & $\begin{array}{l}\text { low } \\
(\mathrm{WER}=1.60-1.75)\end{array}$ \\
\hline $60 \mathrm{~mm}$ & $\begin{array}{l}\text { thickly pachyconic; subevolute } \\
(\mathrm{ww} / \mathrm{dm}=0.75-0.85 ; \mathrm{uw} / \mathrm{dm}=0.30-0.35)\end{array}$ & $\begin{array}{l}\text { strongly depressed; strongly embracing } \\
(\mathrm{ww} / \mathrm{wh}=2.00-2.20 ; \mathrm{IZR}=0.30-0.35)\end{array}$ & $\begin{array}{l}\text { moderate } \\
(\mathrm{WER}=1.80-2.00)\end{array}$ \\
\hline
\end{tabular}

Table 5. Conch dimensions (in $\mathrm{mm}$ ) and proportions for reference specimens of Hammatocyclus corrugatus n. sp.

\begin{tabular}{|c|c|c|c|c|c|c|c|c|c|c|}
\hline & $\mathrm{dm}$ & ww & wh & uw & ah & $w w / d m$ & ww/wh & $\mathrm{uw} / \mathrm{dm}$ & WER & IZR \\
\hline paratype MB.C.18602.2 & 61.5 & 50.0 & 24.2 & 20.1 & 16.9 & 0.81 & 2.06 & 0.33 & 1.90 & 0.30 \\
\hline holotype MB.C.18602.1 & 43.0 & 36.5 & 15.6 & 16.3 & 10.5 & 0.85 & 2.35 & 0.38 & 1.75 & 0.33 \\
\hline paratype MB.C.18602.10 & 40.0 & 32.8 & 12.8 & 16.3 & 9.9 & 0.82 & 2.57 & 0.41 & 1.77 & 0.22 \\
\hline paratype MB.C.18602.3 & 37.3 & 28.3 & 14.1 & 12.6 & 8.8 & 0.76 & 2.00 & 0.34 & 1.71 & 0.38 \\
\hline
\end{tabular}


Table 5. (Continued)

\begin{tabular}{|c|c|c|c|c|c|c|c|c|c|c|}
\hline & $\mathrm{dm}$ & ww & wh & uw & ah & ww/dm & ww/wh & $\mathrm{uw} / \mathrm{dm}$ & WER & IZR \\
\hline paratype MB.C.18602.11 & 36.7 & 30.0 & 14.3 & 14.0 & 9.9 & 0.82 & 2.10 & 0.38 & 1.87 & 0.31 \\
\hline paratype MB.C.18602.4 & 35.5 & 29.1 & 14.4 & 12.6 & 8.7 & 0.82 & 2.03 & 0.35 & 1.75 & 0.40 \\
\hline paratype MB.C.18602.5 & 28.3 & 21.9 & 9.9 & 11.6 & 6.7 & 0.82 & 2.23 & 0.38 & 1.77 & 0.33 \\
\hline paratype MB.C.18602.9 & 26.0 & 22.3 & 9.1 & 10.4 & 5.5 & 0.86 & 2.45 & 0.40 & 1.60 & 0.40 \\
\hline
\end{tabular}

Table 6. Suture line proportions (Figs 9D-F) for Hammatocyclus corrugatus n. sp.

\begin{tabular}{llllllll}
\hline specimen & at dm & EL w/d & EL/VLS & EL/AL & MS h & VLS w/h & remarks \\
\hline holotype MB.C.18602.1 & c. $31.0 \mathrm{~mm}$ & 0.36 & 0.66 & 1.00 & 0.25 & 0.55 & EL slightly concave; AL very narrow \\
paratype MB.C.18602.9 & $23.6 \mathrm{~mm}$ & 0.42 & 0.98 & 0.89 & 0.25 & 0.43 & VLS narrow; AL very asymmetric \\
\hline
\end{tabular}

Discussion. H. corrugatus differs in the wider conch and the wider umbilicus from the type species H. homoceratoides and the co-occurring $H$. pollex. Furthermore, the riblets are usually stronger in $H$. corrugatus than in $H$. pollex. The strength of the riblets is also a good criterion for the separation of the new species from H. confinis Kusina, 1977 and H. incertus Kusina \& Konovalova, 2004. These two species also show a narrower umbilicus than H. corrugatus.
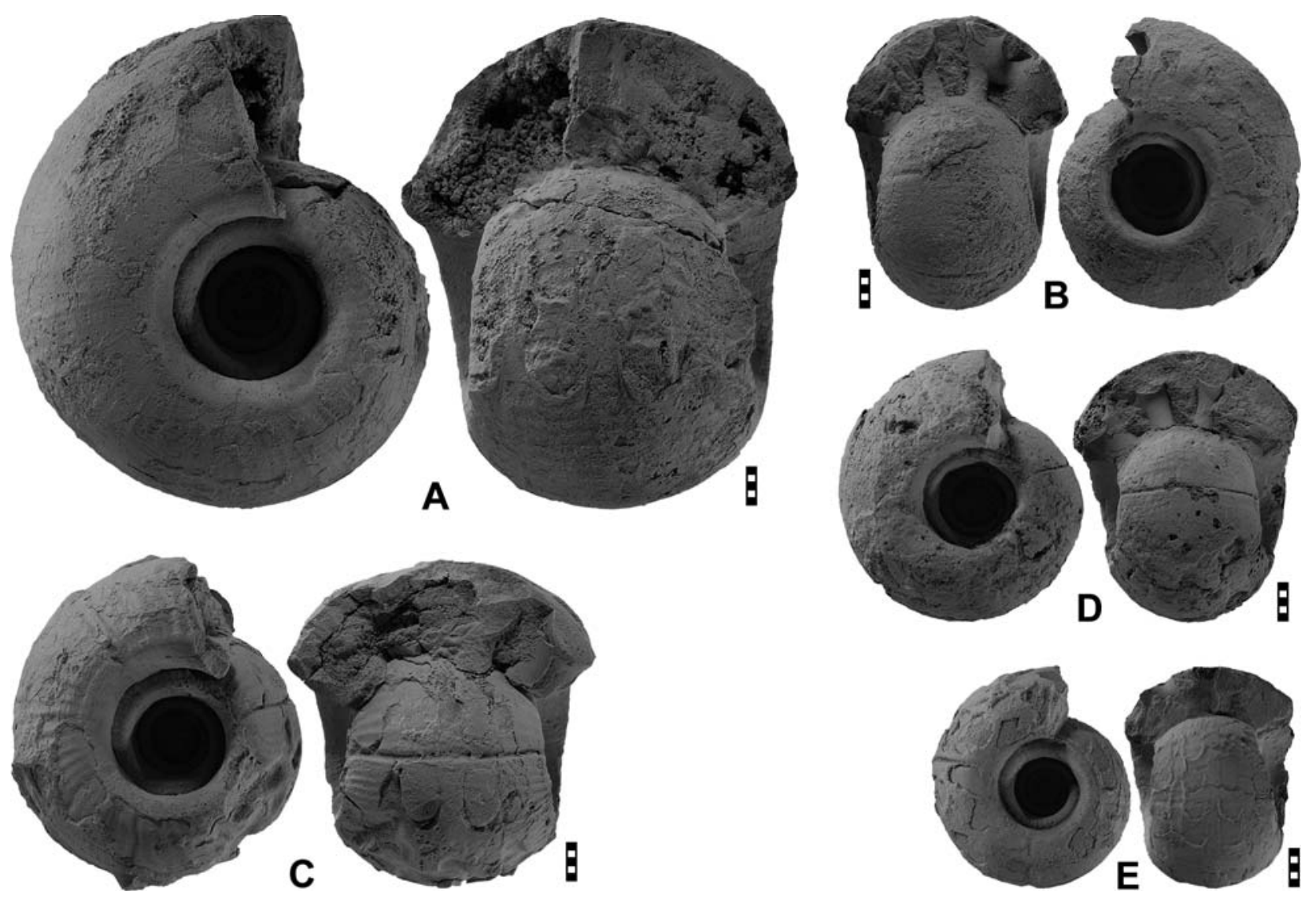

Figure 8. Hammatocyclus corrugatus n. sp. from locality TIM-C7; all $\times 1.0$. A. Paratype MB.C.18602.2. B. Paratype MB.C.18602.3. C. Holotype MB.C.18602.1. D. Paratype MB.C.18602.4. E. Paratype MB.C.18602.5. 

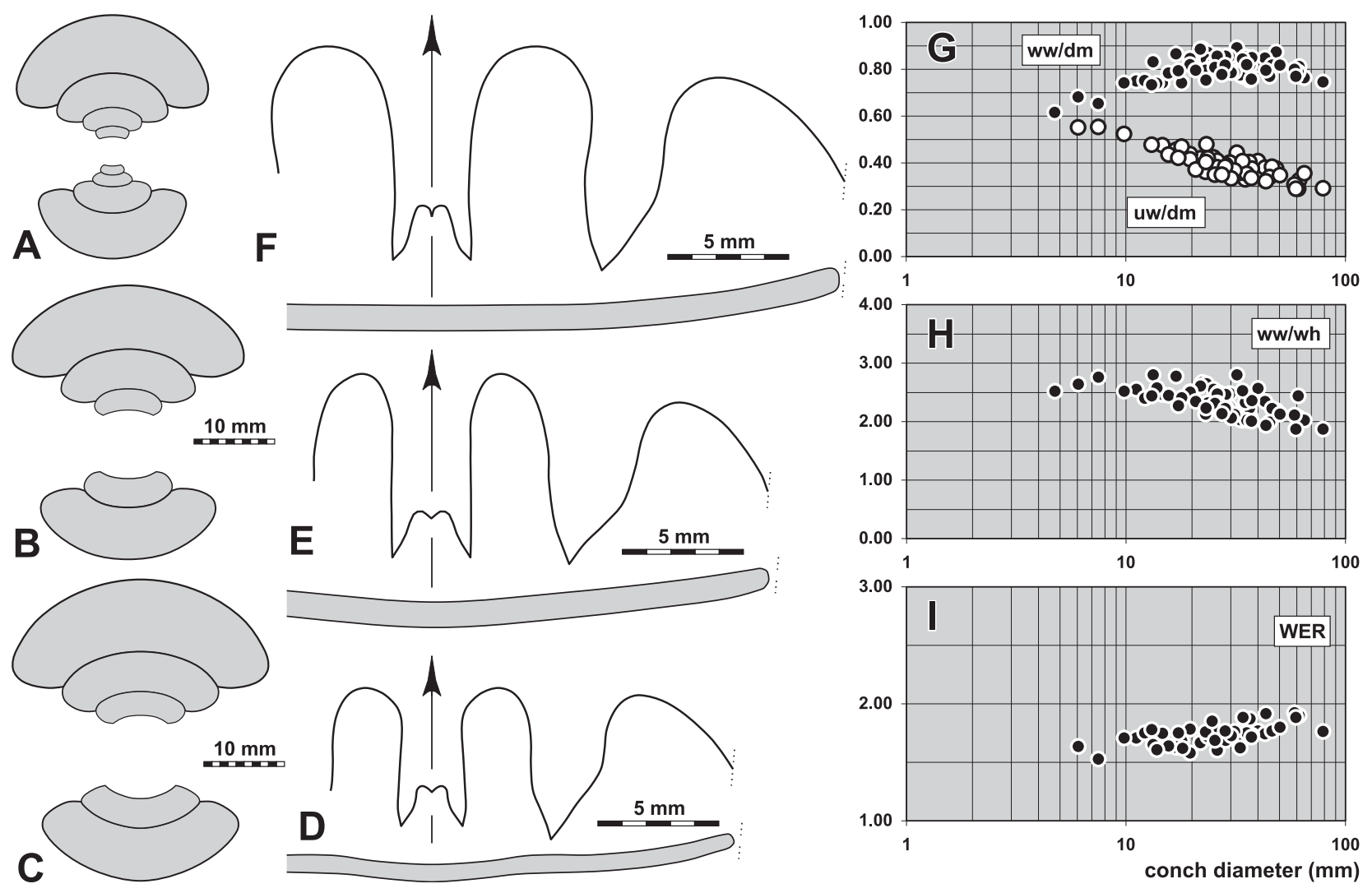

Figure 9. Hammatocyclus corrugatus n. sp. from locality TIM-C7. A. Cross section of paratype MB.C.18602.6; $\times 1.0$. B. Cross section of paratype MB.C.18602.7; $\times 1.0$. C. Cross section of paratype MB.C.18602.8; $\times 1.0$. D. Suture line and constriction of paratype MB.C.18602.5, at $23.1 \mathrm{~mm} \mathrm{dm}, 18.4 \mathrm{~mm} \mathrm{ww}, 7.8 \mathrm{~mm} \mathrm{wh} \times 3.0$. E. Suture line and constriction of paratype MB.C. 18602.9 , at $23.6 \mathrm{~mm} \mathrm{dm}, 20.0 \mathrm{~mm}$ ww, $6.8 \mathrm{~mm}$ wh; $\times 3.0$. F. Suture line and constriction of holotype MB.C.18602.1, at $25.8 \mathrm{~mm}$ ww, $9.7 \mathrm{~mm}$ wh; $\times 3.0$. G-I. Ontogenetic development of the conch width index (ww/dm), umbilical width index (uw/ $\mathrm{dm}$ ), whorl width index (ww/wh), and whorl expansion rate (WER) of all available specimens.

\section{Neopericyclus Popov, 1965}

Type species. Neopericyclus kokdzharensis Popov, 1965 (OD).

Genus definition. Hammatocyclinae with insignificant ontogenetic changes in the conch; adult whorls thickly discoidal and subinvolute to subevolute with moderately high or high aperture; umbilical wall and umbilical margin rounded. Ornament with moderately coarse growth lines or weak riblets, course convex with shallow ventral sinus, direction rectiradiate. Suture line with narrow external lobe; median saddle very low, secondary prongs of the external lobe hook-shaped; adventive lobe V-shaped; dorsolateral saddle strongly asymmetric, narrowly rounded.

Included species.

arenosus: Neopericyclus arenosus n. sp.; Gourara, Algeria.

kokdzharensis: Neopericyclus kokdzharensis Popov, 1965, p. 46; Tien Shan.

moldotooensis: Neopericyclus moldotooensis Popov, 1965, p. 47; Tien Shan.

polaris: Neopericyclus polaris Kusina \& Konovalova, 2004, p. 17; North Urals.

subplicatilis: Pericyclus subplicatilis Crick, 1899, p. 442; Ireland.

\section{Neopericyclus arenosus $\mathbf{n}$. sp.}

Figures 10, 11

Derivation of name. After Lat. arenosus = sandy, because of the position of the type locality between sand dunes.

Holotype. Specimen MB.C.18603.1, illustrated in Figure 10.

Type locality and horizon. Sebkha de Timimoun, locality TIM-C7 (14.5 km west-southwest of Timimoun, Algeria); Ammonellipsites-Merocanites Assemblage.

Material. Two rather well-preserved specimens, the holotype and paratype MB.C.18602.2, of 49 and $52 \mathrm{~mm}$ diameter.

Diagnosis. Neopericyclus with a thinly discoidal and subinvolute conch at $50 \mathrm{~mm} \mathrm{dm}$; whorl cross section weakly depressed; umbilical margin rounded; flanks and venter rounded; aperture moderately high to high. Ornament with moderately coarse growth lines, course convex with shallow ventral sinus, direction rectiradiate; irregularly spaced, faint riblets caused by rhythmic strengthening of the growth lines, delicate nodes on the umbilical margin; five or six constrictions on shell and steinkern, course parallel to the growth lines. Suture line with diverging, slightly 
sinuous flanks of the external lobe; median saddle very low, secondary prongs of the external lobe hook-shaped; adventive lobe V-shaped, asymmetric with gently curved ventral flank and strongly curved dorsal flank; dorsolateral saddle strongly asymmetric, narrowly rounded.

Table 7. Conch dimensions (in $\mathrm{mm}$ ) and proportions for reference specimens of Neopericyclus arenosus $\mathrm{n}$. sp.

\begin{tabular}{llllllllllll}
\hline & $\mathrm{dm}$ & ww & wh & uw & ah & ww/dm & ww/wh & uw/dm & WER & IZR \\
\hline paratype MB.C.18603.2 & 51.9 & 23.3 & 20.9 & 15.0 & 14.6 & 0.45 & 1.11 & 0.29 & 1.94 & 0.30 \\
holotype MB.C.18603.1 & 48.9 & - & 21.0 & 13.8 & 14.8 & - & - & 0.28 & 2.05 & 0.30 \\
& 36.3 & 16.7 & 15.1 & 10.8 & - & 0.46 & 1.11 & 0.30 & - & - \\
\hline
\end{tabular}

Table 8. Suture line proportions (Fig. 11B) for Neopericyclus arenosus n. sp.

\begin{tabular}{lllllll}
\hline specimen & at dm & EL w/d & EL/NLS & EL/AL & MS h & VLS w/h \\
\hline holotype MB.C.18603.1 & c. $42.0 \mathrm{~mm}$ & 0.46 & 0.81 & 0.95 & 0.15 & 0.56 \\
\hline
\end{tabular}

Discussion. Neopericyclus arenosus differs from the type species N. kokdzharensis Popov, 1965 in the weaker and simple riblets (bifurcated in N. kokdzharensis). The median saddle is higher in N. kokdzharensis. N. moldotoensis Popov, 1965 has a slightly wider umbilicus (uw/dm $=0.35$ at $43 \mathrm{~mm} \mathrm{dm}$ ) and a more slender conch (ww/ $\mathrm{dm}=0.40$ ) than $N$. arenosus. $N$. polaris Kusina \& Konovalova, 2004 has a much more compressed conch (ww/ $\mathrm{dm}=0.31$ at $21 \mathrm{~mm} \mathrm{dm})$.
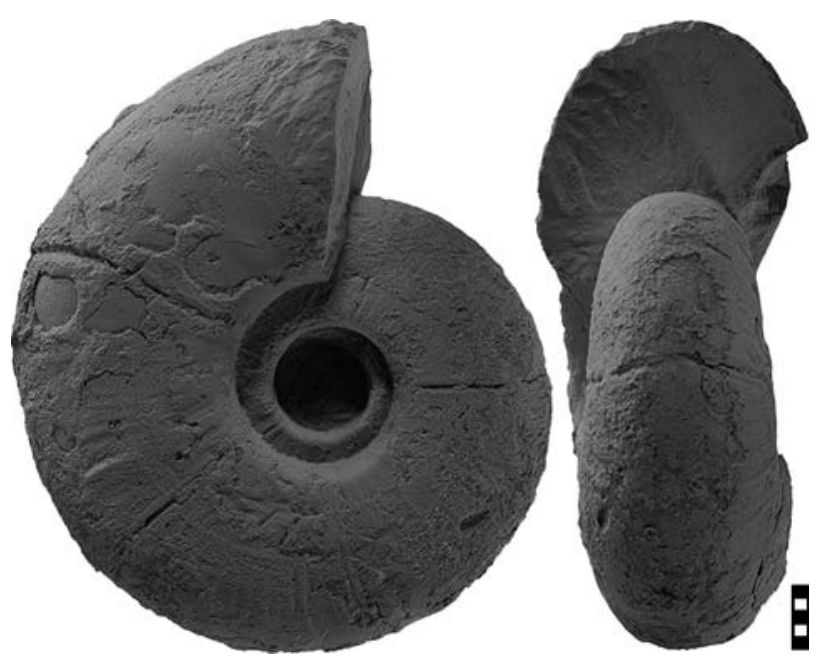

Figure 10. Neopericyclus arenosus n. sp., holotype MB.C.18603.1 from locality TIM-C7; $\times 1$.

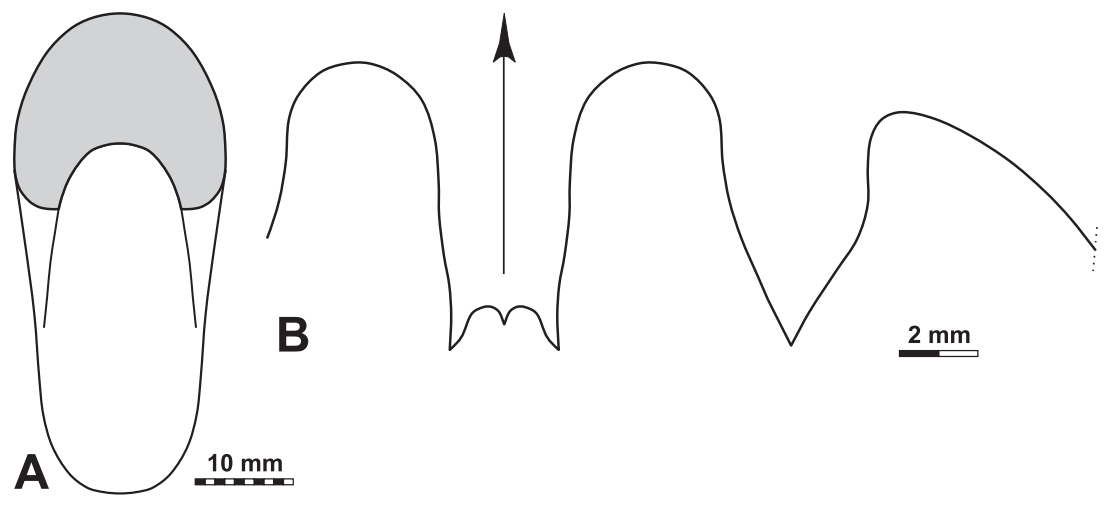

Figure 11. Neopericyclus arenosus n. sp. from locality TIM-C7. A. Dorsal view of holotype MB.C.18603.1; $\times 1.0$. B. Suture line of holotype MB.C.18603.1, at $14.2 \mathrm{~mm}$ ww, $10.2 \mathrm{~mm}$ wh; $\times 4.0$.

Subfamily Ammonellipsitinae Riley, 1996

Subfamily definition. Pericyclidae with insignificant or significant ontogenetic changes of conch geometry; juveniles usually thickly discoidal and subevolute to evolute; adult stage discoidal or pachyconic, subinvolute to subevolute with moderately high or high aperture. Ornament with ribs of various strength. Suture line with narrow V-shaped external lobe with slightly diverging sinuous flanks, median saddle very low or low; ventrolateral saddle rounded to subacute. 
Included genera.

Ammonellipsites Parkinson, 1822

Helicocyclus Schindewolf, 1951

\section{Ammonellipsites Parkinson, 1822}

Type species. Ellipsolithes funatus Sowerby, 1813 (subsequently designated by Schindewolf 1951).

Genus definition. Ammonellipsitinae with significant ontogenetic changes of the conch; inner whorls thickly discoidal and subevolute to evolute; adult whorls discoidal or pachyconic and subinvolute to subevolute (Fig. 12) with high aperture; umbilical wall often flattened, umbilical margin narrowly rounded or subangular. Ornament with sharp and simple concave or slightly biconvex ribs; shell constrictions parallel to the ribs are particularly well-developed in juveniles. Suture line with narrow V-shaped external lobe with slightly diverging flanks, median saddle low. Ventrolateral saddle subacute, adventive lobe V-shaped with gently sinuous flanks.

Included species.

clitheroensis: Ammonellipsites clitheroensis Riley, 1996, p. 70; Lancashire.

conradae: Ammonellipsites conradae n. sp.; Gourara, Algeria.

djaprakensis: Pericyclus djaprakensis Librovitch, 1927, p. 17; Tien Shan.

doohylensis: Pericyclus Doohylensis Foord \& Crick, 1897, p. 151; Ireland.

funatus: Ellipsolithes funatus Sowerby, 1813, p. 81; Ireland.

grandicostatus: Pericyclus grandicostatus Librovitch, 1927, p. 23; Tien Shan.

hauchecornei: Pericyclus Hauchecornei Holzapfel, 1889, p. 36; Rhenish Mountains.

inflatus: Pericyclus inflatus Librovitch, 1927, p. 14; Tien Shan. [Synonym of p. nikitini]

kayseri: Pericyclus Kayseri Schmidt, 1925, p. 554; Rhenish Mountains.

kochi: Pericyclus Kochi Holzapfel, 1889, p. 35; Rhenish Mountains.

menchikoffi: Ammonellipsites menchikoffi n. sp; Gourara, Algeria.

multicostatus: Pericyclus multicostatus Foord, 1901, p. 139; Ireland.

nikitini: Pericyclus Nikitini Librovitch, 1927, p. 14; Tien Shan.

pareyni: Ammonellipsites pareyni n. sp.; Gourara, Algeria.

sguilmensis: Ammonellipsites sguilmensis Korn, Ebbighausen \& Bockwinkel, 2010; Saoura Valley, Algeria.

sonkulensis: Pericyclus sonkulensis Librovitch, 1927, p. 17; Tien Shan. [Synonym of p.djaprakensis]

subcompressus: Pericyclus subcompressus Librovitch, 1927, p. 14; Tien Shan. [Synonym of p. nikitini]

subglaber: Pericyclus subglaber Holzapfel, 1889, p. 37; Rhenish Mountains.

Separation of the new species. The three species of Ammonellipsites from the Dalle à Merocanites can easily be separated by their conch morphology. In the conch shape, specimens with $30 \mathrm{~mm}$ diameter are characterised as follows:

- Ammonellipsites pareyni - conch thickly discoidal, subinvolute $(\mathrm{ww} / \mathrm{dm}=0.50-0.55 ; \mathrm{uw} / \mathrm{dm}=0.15-0.20)$; aperture high $(\mathrm{WER}=2.25-$ $2.50)$

- Ammonellipsites menchikoffi - conch thickly discoidal, subinvolute or subevolute $(\mathrm{ww} / \mathrm{dm}=0.50-0.60 ; \mathrm{uw} / \mathrm{dm}=0.27-0.33)$; aperture high (WER = 1.75-1.90);

- Ammonellipsites conradae - conch thinly discoidal, subevolute $(\mathrm{ww} / \mathrm{dm}=0.40-0.45$; uw/dm $=0.38-0.43)$; aperture low $(\mathrm{WER}=1.55-$ $1.75)$.
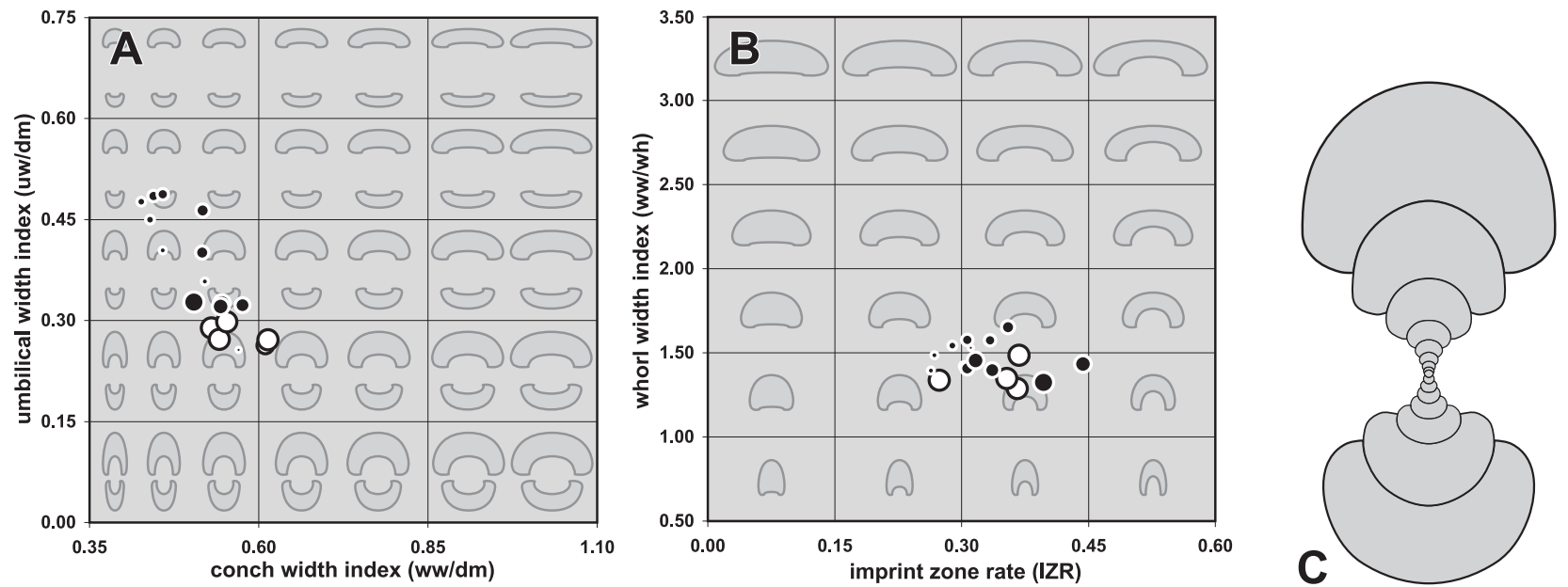

Figure 12. Ontogenetic trajectories of Ammonellipsites, exemplified for Ammonellipsites menchikoffi n. sp. from locality TIM-C7. A. Ontogenetic development of the conch width index (ww/dm), umbilical width index (uw/dm). B. Ontogenetic development of the imprint zone rate (IZR), whorl width index (ww/wh). C. Cross section of paratype MB.C.18605.6; $\times 2.0$. [Black dots represent ontogenetic stages of cross section MB.C.18605.6, white dots represent the reference specimens (Tab. 13).] 
Discussion. Ammonellipsites differs from Fascipericyclus Turner, 1948 in the simple ribs (frequently bifurcated or intercalated in Fascipericyclus). Helicocyclus Schindewolf, 1951 has a much wider umbilicus and less rapidly increasing apertural height.

\section{Ammonellipsites pareyni $\mathrm{n} . \mathbf{s p}$.}

Figures 13, 14

1984 Ammonellipsites kocki. - Conrad, pl. 5, fig. 8.

Derivation of name. After Claude Pareyn, in honour of his contribution to Early Carboniferous stratigraphy in Algeria.

Holotype. Specimen MB.C.18604.1, illustrated in Figure 13E.

Type locality and horizon. Sebkha de Timimoun, locality TIM-C7 (14.5 km west-southwest of Timimoun, Algeria); Ammonellipsites-Merocanites Assemblage.

Material. A total of 60 specimens between 12 and $33 \mathrm{~mm}$ conch diameter are available. The maximum conch diameter can be estimated to be about $50 \mathrm{~mm}$. Most of the specimens are rather strongly corroded phragmocones; shell remains are frequently preserved.

Diagnosis. Ammonellipsites with a subinvolute conch, changing from thinly pachyconic to thickly discoidal between 10 and $30 \mathrm{~mm}$ dm; aperture moderately high in juveniles and rapidly becoming very high during ontogeny. Ornament with $65-70$ sharp equidistant ribs per volution, course slightly biconvex and rectiradiate with barely visible lateral sinus and very shallow ventral sinus. Suture line with narrow, V-shaped external lobe with gently sinuous flanks and low median saddle; ventrolateral saddle narrowly rounded, adventive lobe V-shaped, asymmetric.

Table 9. Conch ontogeny (Figs 14A-C, E-G) of Ammonellipsites pareyni n. sp.

\begin{tabular}{|c|c|c|c|}
\hline & conch shape & whorl cross section shape & aperture \\
\hline $4 \mathrm{~mm}$ & $\begin{array}{l}\text { thickly discoidal; subevolute } \\
(\mathrm{ww} / \mathrm{dm}=0.52-0.60 ; \mathrm{uw} / \mathrm{dm}=0.30-0.40)\end{array}$ & $\begin{array}{l}\text { weakly depressed to moderately depressed; strongly } \\
\text { embracing ( } w w / w h=1.45-1.65 ; \text { IZR }=0.30-0.36 \text { ) }\end{array}$ & $\begin{array}{l}\text { moderate } \\
(\mathrm{WER}=1.80-1.90)\end{array}$ \\
\hline $10 \mathrm{~mm}$ & $\begin{array}{l}\text { thinly pachyconic; subinvolute } \\
(\mathrm{ww} / \mathrm{dm}=0.65-0.70 ; \mathrm{uw} / \mathrm{dm}=0.17-0.22 \text { ) }\end{array}$ & $\begin{array}{l}\text { weakly depressed; strongly embracing } \\
(\mathrm{ww} / \mathrm{wh}=1.35-1.50 ; \mathrm{IZR}=0.30-0.40)\end{array}$ & $\begin{array}{l}\text { moderate to high } \\
(\mathrm{WER}=1.90-2.20)\end{array}$ \\
\hline $30 \mathrm{~mm}$ & $\begin{array}{l}\text { thickly discoidal; subinvolute } \\
(\mathrm{ww} / \mathrm{dm}=0.50-0.55 ; \mathrm{uw} / \mathrm{dm}=0.15-0.20 \text { ) }\end{array}$ & $\begin{array}{l}\text { weakly depressed; moderately to strongly embracing } \\
\text { (ww/wh }=1.15-1.30 ; \mathrm{IZR}=0.25-0.35 \text { ) }\end{array}$ & $\begin{array}{l}\text { high } \\
(\text { WER }=2.25-2.50)\end{array}$ \\
\hline
\end{tabular}
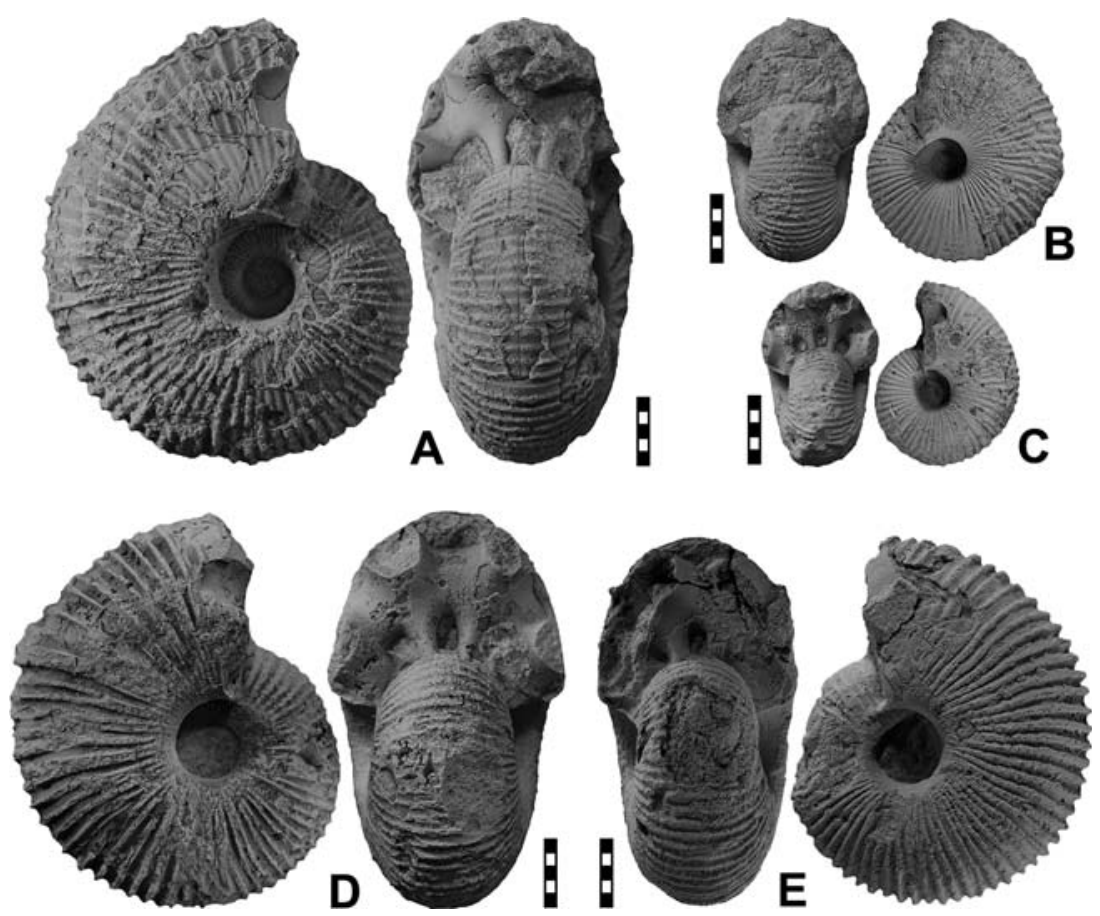

Figure 13. Ammonellipsites pareyni $\mathrm{n}$. sp. from locality TIM-C7; all $\times 1.5$. A. Paratype MB.C.18604.2. B. Paratype MB.C.18604.3. C. Paratype MB.C.18604.4. D. Paratype MB.C.18604.5. E. Holotype MB.C.18604.1. 
Table 10. Conch dimensions (in mm) and proportions for reference specimens of Ammonellipsites pareyni $\mathrm{n}$. sp.

\begin{tabular}{|c|c|c|c|c|c|c|c|c|c|c|}
\hline & $\mathrm{dm}$ & ww & wh & uw & ah & $w w / d m$ & ww/wh & $\mathrm{uw} / \mathrm{dm}$ & WER & IZR \\
\hline paratype MB.C.18604.5 & 28.3 & 16.7 & 13.7 & 5.6 & 9.2 & 0.59 & 1.22 & 0.20 & 2.19 & 0.33 \\
\hline holotype MB.C.18604.1 & 25.7 & 14.3 & 12.0 & 4.9 & 8.7 & 0.56 & 1.19 & 0.19 & 2.29 & 0.28 \\
\hline paratype MB.C.18604.9 & 23.1 & 13.4 & 11.0 & 5.3 & 7.2 & 0.58 & 1.22 & 0.23 & 2.12 & 0.34 \\
\hline paratype MB.C.18604.3 & 17.1 & 10.5 & 9.2 & 2.9 & 5.7 & 0.62 & 1.15 & 0.17 & 2.26 & 0.38 \\
\hline paratype MB.C.18604.4 & 13.0 & 8.2 & 6.9 & 2.5 & 4.3 & 0.63 & 1.19 & 0.19 & 2.25 & 0.37 \\
\hline
\end{tabular}

Table 11. Suture line proportions (Fig. 14D) for Ammonellipsites pareyni n. sp.

\begin{tabular}{llllllll}
\hline specimen & at dm & EL w/d & EL/VLS & EL/AL & MS h & VLS w/h \\
\hline paratype MB.C.18604.2 & $31.2 \mathrm{~mm}$ & 0.55 & 1.34 & 1.16 & 0.34 & 0.41 \\
\hline
\end{tabular}

Discussion. Ammonellipsites pareyni belongs to the narrowly umbilicate species of the genus; it has a narrower umbilicus and a higher aperture than the co-occurring species $A$. menchikoffi and $A$. conradae. The differences are particularly obvious in specimens of $25-30 \mathrm{~mm}$ conch diameter, where A. pareyni shows a slower opening of the umbilicus $(\mathrm{uw} / \mathrm{dm}=0.20)$ and a higher aperture $(\mathrm{WER}=2.25)$. A. menchikoffi shows a rather rapid opening of the umbilicus in this growth stage $(\mathrm{uw} / \mathrm{dm}=0.25-0.30)$ with a low or moderately high aperture $(\mathrm{WER}=1.65-$ 1.85).

A. clitheroensis Riley, 1996, A. doohylensis (Foord \& Crick, 1897), and A. multicostatus (Foord, 1901) have also a rather narrow umbilicus like $A$. pareyni, but possess a pachyconic, much wider conch $(\mathrm{ww} / \mathrm{dm}=0.70)$ than A. pareyni $(\mathrm{ww} / \mathrm{dm}=0.55-0.60$ at $20 \mathrm{~mm} \mathrm{dm})$. A. nikitini (Librovitch, 1927) has also a much stouter conch.
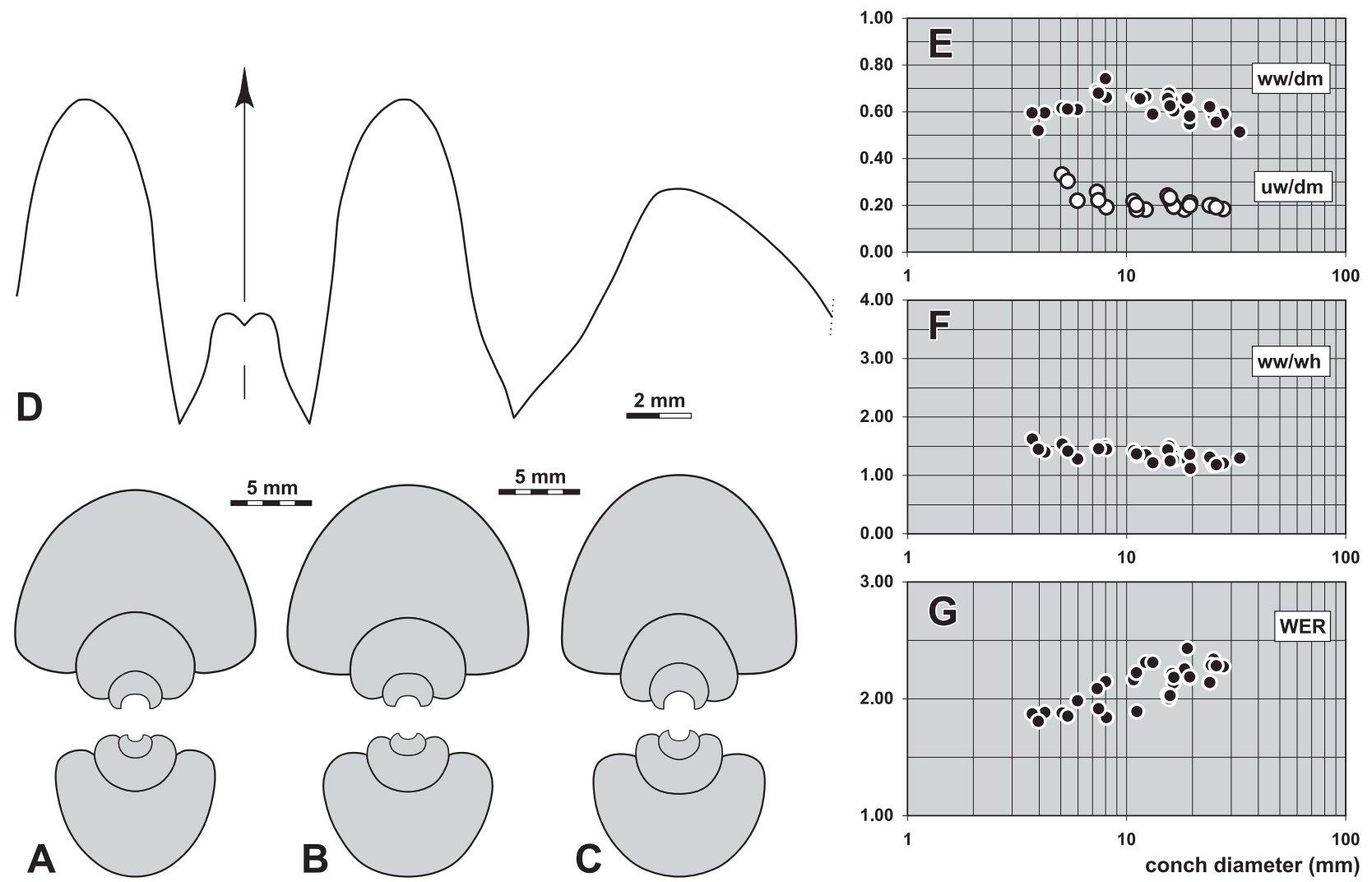

Figure 14. Ammonellipsites pareyni n. sp. from locality TIM-C7. A. Cross section of paratype MB.C.18604.6; $\times 2.0$. B. Cross section of paratype MB.C.18604.7; $\times 2.0$. C. Cross section of paratype MB.C.18604.8; $\times 2.0$. D. Suture line of paratype MB.C.18604.2, at $31.2 \mathrm{~mm} \mathrm{dm}, 14.8 \mathrm{~mm}$ wh; $\times 4.0$. E-G. Ontogenetic development of the conch width index (ww/dm), umbilical width index (uw/dm), whorl width index (ww/wh), and whorl expansion rate (WER) of all available specimens. 
Of the species known from the Rhenish Mountains, A. kochi (Holzapfel, 1889) has a wider conch (ww/ $\mathrm{dm}=0.70$ at $35 \mathrm{~mm} \mathrm{dm}$ ) and much stronger ribs in juveniles, A. hauchecornei (Holzapfel, 1889) has a much wider umbilicus (uw/dm $=0.35$ at $26 \mathrm{~mm} \mathrm{dm}$ ), and A. kayseri (Schmidt, 1925) has much finer riblets (approximately 100 or more per volution).

\section{Ammonellipsites menchikoffi n. sp.}

Figures 12, 15, 16

Derivation of name. After Nicolas Menchikoff, in honour of his contribution to Early Carboniferous stratigraphy in Algeria.

Holotype. Specimen MB.C.18605.1, illustrated in Figure 15D.

Type locality and horizon. Sebkha de Timimoun, locality TIM-C7 (14.5 km west-southwest of Timimoun, Algeria); Ammonellipsites-Merocanites Assemblage.

Material. Ten specimens between 21 and $33 \mathrm{~mm}$ conch diameter are available. Some of the larger specimens are fully septate, and hence a maximum conch diameter of about $50 \mathrm{~mm}$ can be estimated. The specimens are rather strongly corroded phragmocones, but shell remains are occasionally preserved.

Diagnosis. Ammonellipsites with a thickly discoidal, subevolute conch between 10 and $30 \mathrm{~mm}$ dm; aperture very low in juveniles and rapidly becoming moderately high during ontogeny. Ornament with 80 sharp equidistant riblets per volution, course almost linear across flanks and venter, direction slightly rursiradiate. Suture line with very narrow or narrow, V-shaped external lobe with slightly sinuous flanks and low median saddle; ventrolateral saddle almost asymmetric, narrowly rounded or subacute; adventive lobe slightly asymmetric Vshaped.

Table 12. Conch ontogeny (Figs 16A, D-F) of Ammonellipsites menchikoffi n. sp.

\begin{tabular}{|c|c|c|c|}
\hline$d m$ & conch shape & whorl cross section shape & aperture \\
\hline $4 \mathrm{~mm}$ & $\begin{array}{l}\text { thinly discoidal; evolute } \\
\text { (ww/dm } \sim 0.45 ; \text { uw/dm } \sim 0.48 \text { ) }\end{array}$ & $\begin{array}{l}\text { moderately depressed; strongly embracing } \\
\text { (ww/wh } \sim 1.55 ; \text { IZR } \sim 0.32 \text { ) }\end{array}$ & $\begin{array}{l}\text { low } \\
(\text { WER } \sim 1.55)\end{array}$ \\
\hline $10 \mathrm{~mm}$ & $\begin{array}{l}\text { thickly discoidal; subevolute } \\
\text { (ww/dm } \sim 0.55 ; \text { uw/dm } \sim 0.35 \text { ) }\end{array}$ & $\begin{array}{l}\text { weakly depressed; strongly embracing } \\
\text { (ww/wh } \sim 1.40 ; \text { IZR } \sim 0.32 \text { ) }\end{array}$ & $\begin{array}{l}\text { moderate } \\
(\text { WER } \sim 1.85)\end{array}$ \\
\hline 30 mm & $\begin{array}{l}\text { thickly discoidal; subinvolute to subevolute } \\
\text { (ww/dm }=0.50-0.60 ; \mathrm{uw} / \mathrm{dm}=0.27-0.33 \text { ) }\end{array}$ & $\begin{array}{l}\text { weakly depressed; strongly embracing } \\
\text { (ww/wh }=1.25-1.50 ; \text { IZR }=0.35-0.40 \text { ) }\end{array}$ & $\begin{array}{l}\text { moderate } \\
(\mathrm{WER}=1.75-1.90)\end{array}$ \\
\hline
\end{tabular}
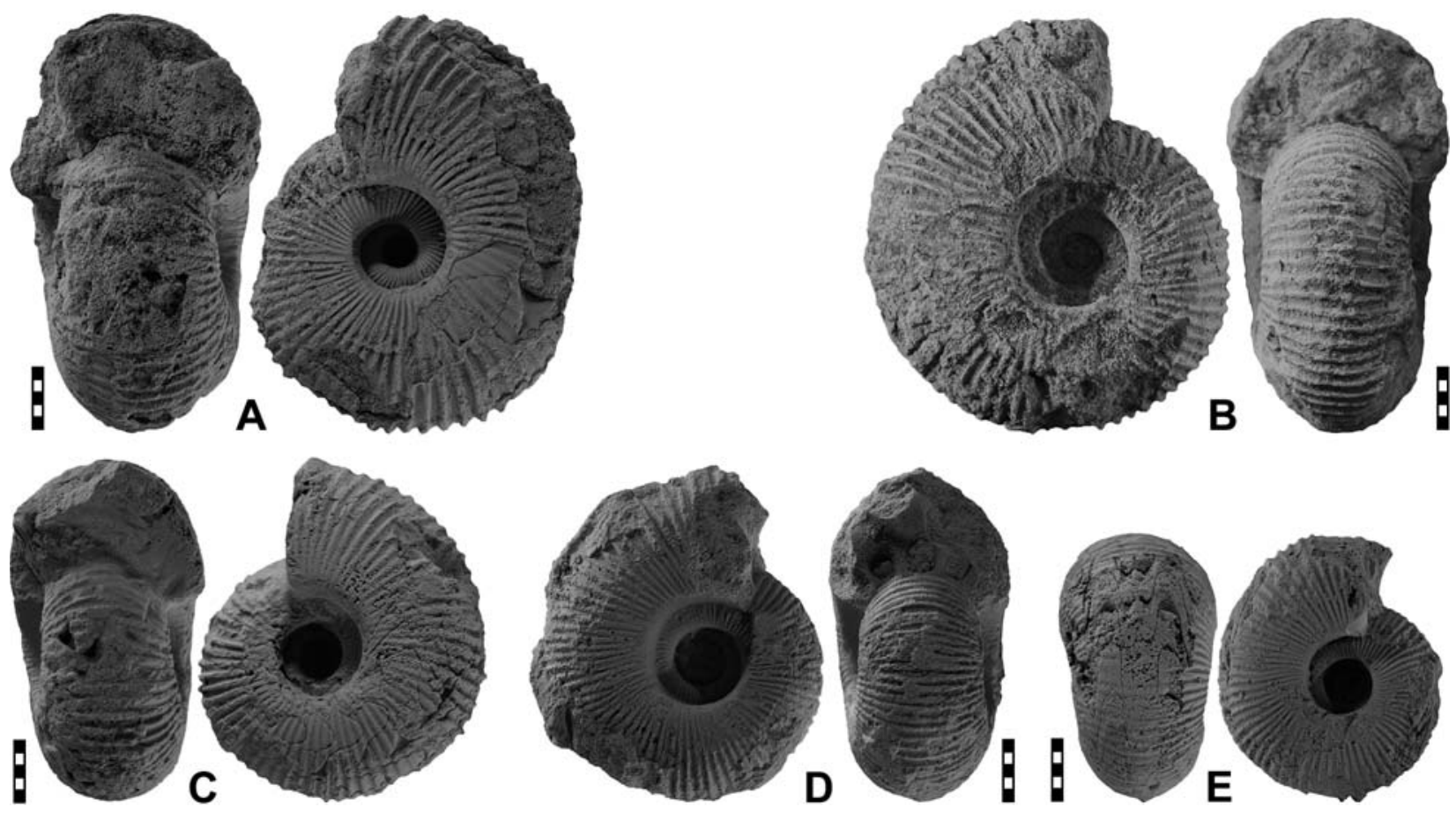

Figure 15. Ammonellipsites menchikoffi n. sp. from locality TIM-C7; all $\times 1.5$. A. Paratype MB.C.18605.2. B. Paratype MB.C.18605.3. C. Paratype MB.C.18605.4. D. Holotype MB.C.18605.1. E. Paratype MB.C.18605.5. 
Table 13. Conch dimensions (in mm) and proportions for reference specimens of Ammonellipsites menchikoffi $\mathrm{n}$. sp.

\begin{tabular}{lccccccccccc}
\hline & $\mathrm{dm}$ & ww & wh & uw & ah & ww/dm & ww/wh & uw/dm & WER & IZR \\
\hline paratype MB.C.18605.3 & 32.4 & 17.2 & 12.8 & 9.4 & 9.3 & 0.53 & 1.34 & 0.29 & 1.97 & 0.27 \\
paratype MB.C.18605.2 & 32.3 & 19.8 & 13.3 & 8.8 & 8.4 & 0.61 & 1.49 & 0.27 & 1.83 & 0.37 \\
paratype MB.C.18605.4 & 26.5 & 14.3 & 11.1 & 7.2 & 7.0 & 0.54 & 1.29 & 0.27 & 1.86 & 0.37 \\
holotype MB.C.18605.1 & 26.0 & 14.4 & 10.6 & 7.8 & 6.9 & 0.55 & 1.35 & 0.30 & 1.85 & 0.35 \\
paratype MB.C.18605.5 & 21.4 & 13.1 & 8.8 & 5.6 & 5.6 & 0.61 & 1.48 & 0.26 & 1.82 & 0.37 \\
\hline
\end{tabular}

Table 14. Suture line proportions (Figs 16B, C) for Ammonellipsites menchikoffi n. sp.

\begin{tabular}{|c|c|c|c|c|c|c|}
\hline specimen & at $\mathrm{dm}$ & $E L w / d$ & EL/VLS & $\mathrm{EL} / \mathrm{AL}$ & MS h & VLS w/h \\
\hline paratype MB.C.18605.4 & $19.3 \mathrm{~mm}$ & 0.50 & 1.07 & 1.19 & 0.30 & 0.46 \\
\hline paratype MB.C. 18605.5 & $16.2 \mathrm{~mm}$ & 0.50 & 1.14 & 1.10 & 0.27 & 0.43 \\
\hline
\end{tabular}

Discussion. Ammonellipsites menchikoffi has a morphological position between the co-occurring species $A$. pareyni (which is narrower umbilicate) and A. conradae (which has a wider umbilicus). A. clitheroensis Riley, 1996, A. kochi (Holzapfel, 1889), and A. kayseri (Schmidt, 1925) have a much narrower umbilicus. A. hauchecornei (Holzapfel, 1889) has a similar conch but possesses weaker, less sharp riblets (about 70 per volution) and a slightly more slender conch $(\mathrm{ww} / \mathrm{dm}=0.45$ at $28 \mathrm{~mm} \mathrm{dm})$.
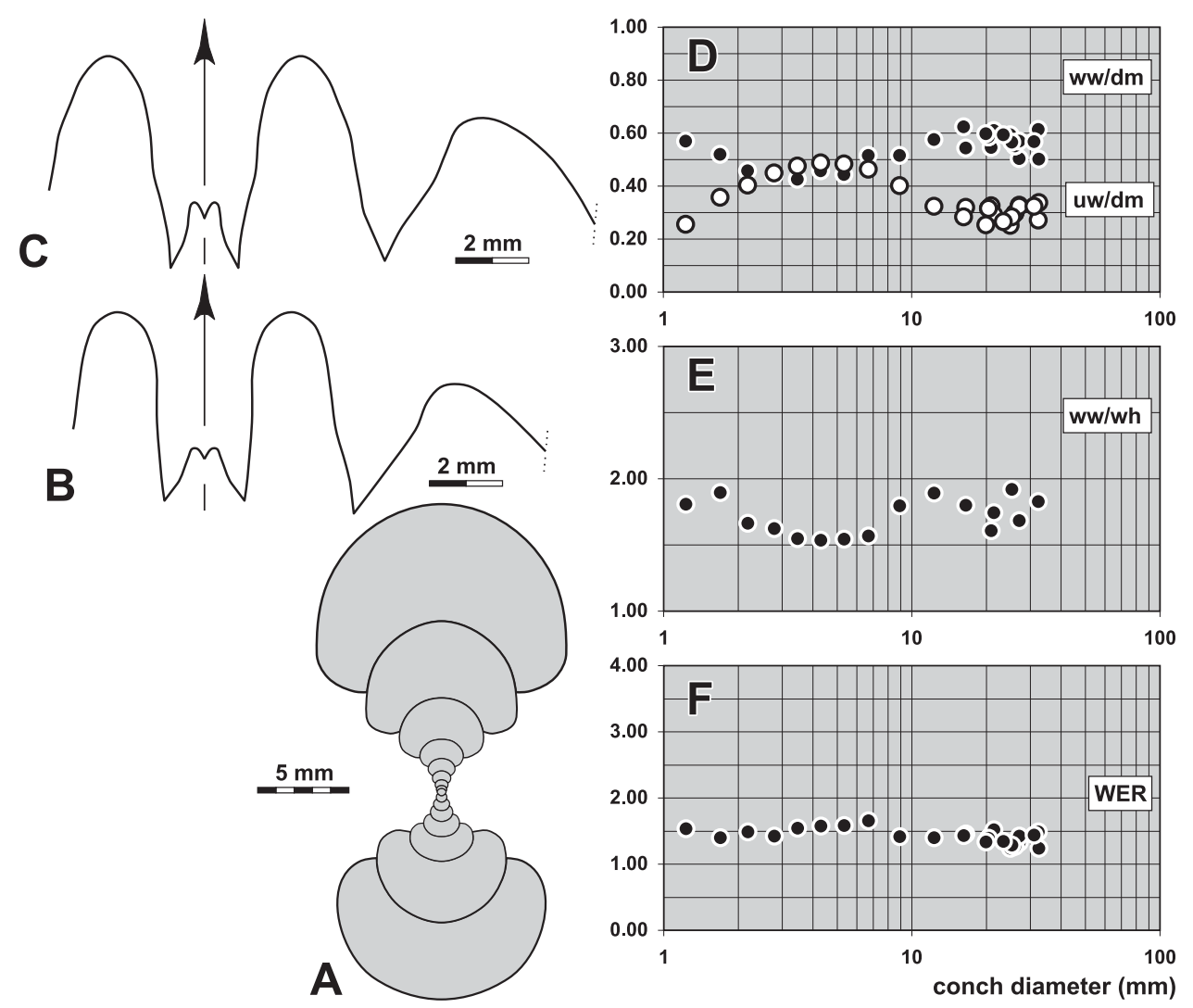

Figure 16. Ammonellipsites menchikoffi $\quad$ n. sp. from locality TIM-C7. A. Cross section of paratype MB.C.18605.6; × 2.0. B. Suture line of paratype $\mathrm{B} 057$, at $16.2 \mathrm{~mm} \mathrm{dm}$, $10.2 \mathrm{~mm}$ ww, $7.5 \mathrm{~mm}$ wh; $\times 4.0$. C. Suture line of paratype B058, at $19.3 \mathrm{~mm}$ $\mathrm{dm}, 11.3 \mathrm{~mm}$ ww, $7.7 \mathrm{~mm}$ wh; $\times 4.0$. D-F. Ontogenetic development of the conch width index (ww/ $\mathrm{dm})$, umbilical width index (uw/dm), whorl width index (ww/wh), and whorl expansion rate (WER) of all available specimens.

Ammonellipsites conradae n. sp.

Figures 17, 18

Derivation of name. After Jacqueline Conrad, in honour of her contribution to Early Carboniferous stratigraphy in Algeria.

Holotype. Specimen MB.C.18606.1, illustrated in Figure 17B.

Type locality and horizon. Sebkha de Timimoun, locality TIM-C7 (14.5 km west-southwest of Timimoun, Algeria); Ammonellipsites-Merocanites Assemblage.

Material. Four specimens between 25 and $34 \mathrm{~mm}$ conch diameter are available. The maximum conch diameter of about $45 \mathrm{~mm}$ can be estimated. The specimens are rather strongly corroded; shell remains are poorly preserved. 
Diagnosis. Ammonellipsites with a thickly discoidal, subevolute conch between 10 and $30 \mathrm{~mm}$ dm; aperture low throughout ontogeny. Ornament with 65 sharp equidistant riblets per volution, course convex with shallow ventral sinus, direction rursiradiate. Suture line with narrow, V-shaped external lobe and low median saddle; flanks of the external lobe slightly sinuous; ventrolateral saddle almost asymmetric, narrowly rounded; adventive lobe slightly asymmetric V-shaped.

Table 15. Conch ontogeny (Figs 18A, C-E) of Ammonellipsites conradae n. sp.

\begin{tabular}{|c|c|c|c|}
\hline$d m$ & conch shape & whorl cross section shape & aperture \\
\hline \multirow[t]{2}{*}{$5 \mathrm{~mm}$} & discoidal; evolute & moderately depressed; strongly embracing & low \\
\hline & $(\mathrm{ww} / \mathrm{dm} \sim 0.48 ; \mathrm{uw} / \mathrm{dm} \sim 0.50)$ & $($ ww/wh $\sim 1.70 ;$ IZR 0.30) & $($ WER $\sim 1.50)$ \\
\hline \multirow[t]{2}{*}{$10 \mathrm{~mm}$} & thickly discoidal; subevolute & moderately depressed; strongly embracing & low \\
\hline & $(\mathrm{ww} / \mathrm{dm} \sim 0.54 ; \mathrm{uw} / \mathrm{dm} \sim 0.42)$ & $($ ww/wh $\sim 1.65 ;$ IZR 0.30) & $($ WER 1.70) \\
\hline \multirow[t]{2}{*}{$30 \mathrm{~mm}$} & thinly discoidal; subevolute & weakly depressed; strongly embracing & low \\
\hline & $(w w / d m=0.40-0.45 ; u w / d m=0.38-0.43)$ & $(w w / w h=1.30-1.40 ;$ IZR $=0.30-0.35)$ & $($ WER $=1.55-1.75)$ \\
\hline
\end{tabular}

Table 16. Conch dimensions (in mm) and proportions for reference specimens of Ammonellipsites conradae n. sp.

\begin{tabular}{lllllllllll}
\hline & dm & ww & wh & uw & ah & ww/dm & ww/wh & uw/dm & WER & IZR \\
\hline paratype MB.C.18606.2 & 33.7 & - & 12.1 & 13.9 & 8.4 & - & - & 0.41 & 1.77 & 0.31 \\
paratype MB.C.18606.4 & 30.5 & - & 9.9 & 12.9 & 6.4 & - & - & 0.42 & 1.60 & 0.36 \\
holotype MB.C.18606.1 & 25.7 & 10.8 & 8.7 & 11.1 & 5.7 & 0.42 & 1.24 & 0.43 & 1.65 & 0.34 \\
\hline
\end{tabular}

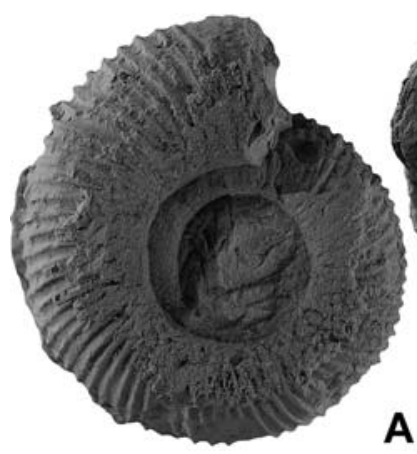

A

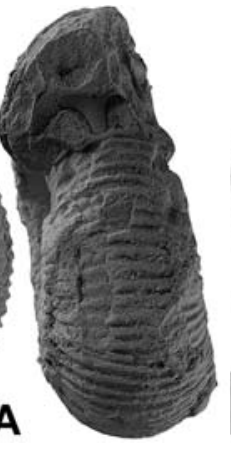

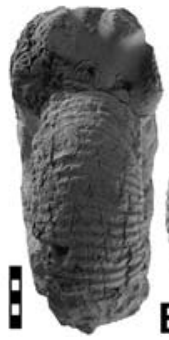

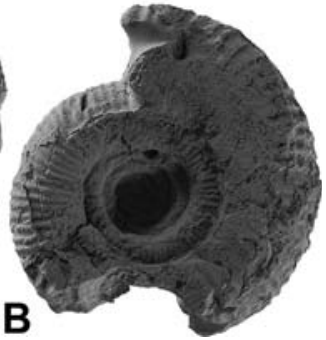

Figure 17. Ammonellipsites conradae n. sp. from locality TIM-C7; all $\times 1.5$. A. Paratype MB.C.18606.2. B. Holotype MB.C.18606.1.
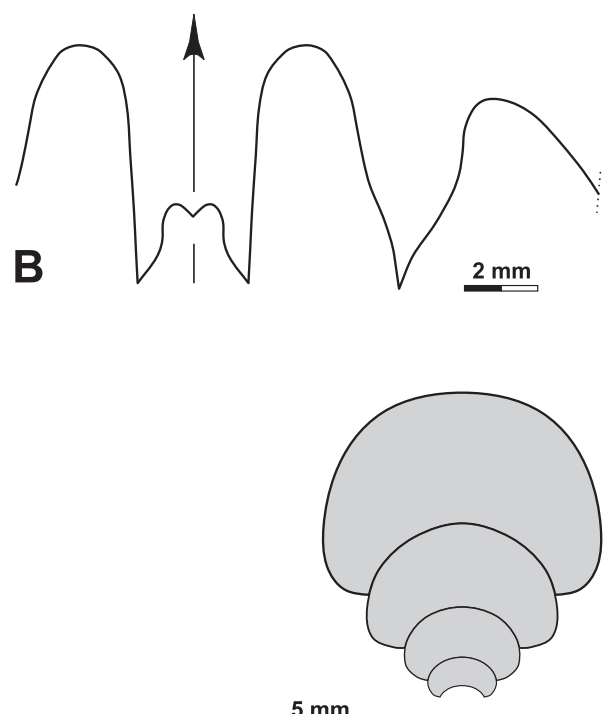

$5 \mathrm{~mm}$

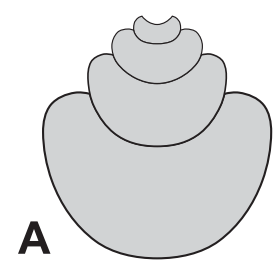

1.00
0.80
0.60
0.40
0.20
0.00

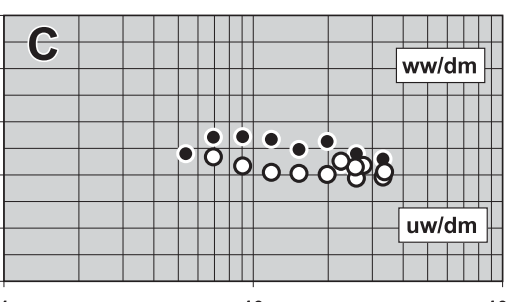

10
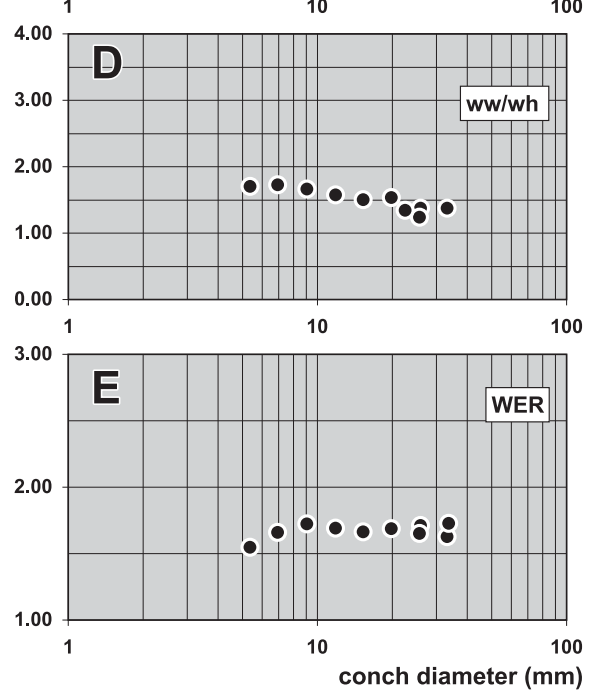

Figure 18. Ammonellipsites conradae $\mathrm{n}$. $\mathrm{sp}$. from locality TIMC7. A. Cross section of paratype MB.C.18606.3; $\times 2.0$. B. Suture line of holotype MB.C.18606.1, at $23.1 \mathrm{~mm} \mathrm{dm}$, $10.6 \mathrm{~mm}$ ww, $7.8 \mathrm{~mm}$ wh; $\times 4.0$. C-E. Ontogenetic development of the conch width index (ww/ $\mathrm{dm}$ ), umbilical width index (uw/ $\mathrm{dm}$ ), whorl width index (ww/ wh), and whorl expansion rate (WER) of all available specimens. 
Table 17. Suture line proportions (Fig. 18B) for Ammonellipsites conradae n. sp.

\begin{tabular}{lllllll}
\hline specimen & at dm & EL w/d & EL/vLS & EL/AL & MS h \\
\hline holotype MB.C.18606.1 & $23.1 \mathrm{~mm}$ & 0.53 & 1.16 & 1.32 & 0.33 \\
\hline
\end{tabular}

Discussion. Ammonellipsites conradae belongs to the widely umbilicate species within the genus; it is the widest umbilicate of the three Ammonellipsites species found in the Dalle à Merocanites. At $25 \mathrm{~mm}$ conch diameter, its uw/dm ratio is higher than 0.40 ; at the same size this ratio amounts only to 0.30 in $A$. menchikoffi and to 0.20 in A. pareyni.

Similarly widely umbilicate species like $A$. conradae are $A$. funatus (Sowerby, 1813), A. djaprakensis (Librovitch, 1927), and A. tianshanicus (Librovitch, 1927), but these possess rather strong constrictions in the adult stage.

Family Muensteroceratidae Librovitch, 1957

\section{Muensteroceras Hyatt, 1884}

For a detailed discussion of the genus, see Korn et al. (2010a).
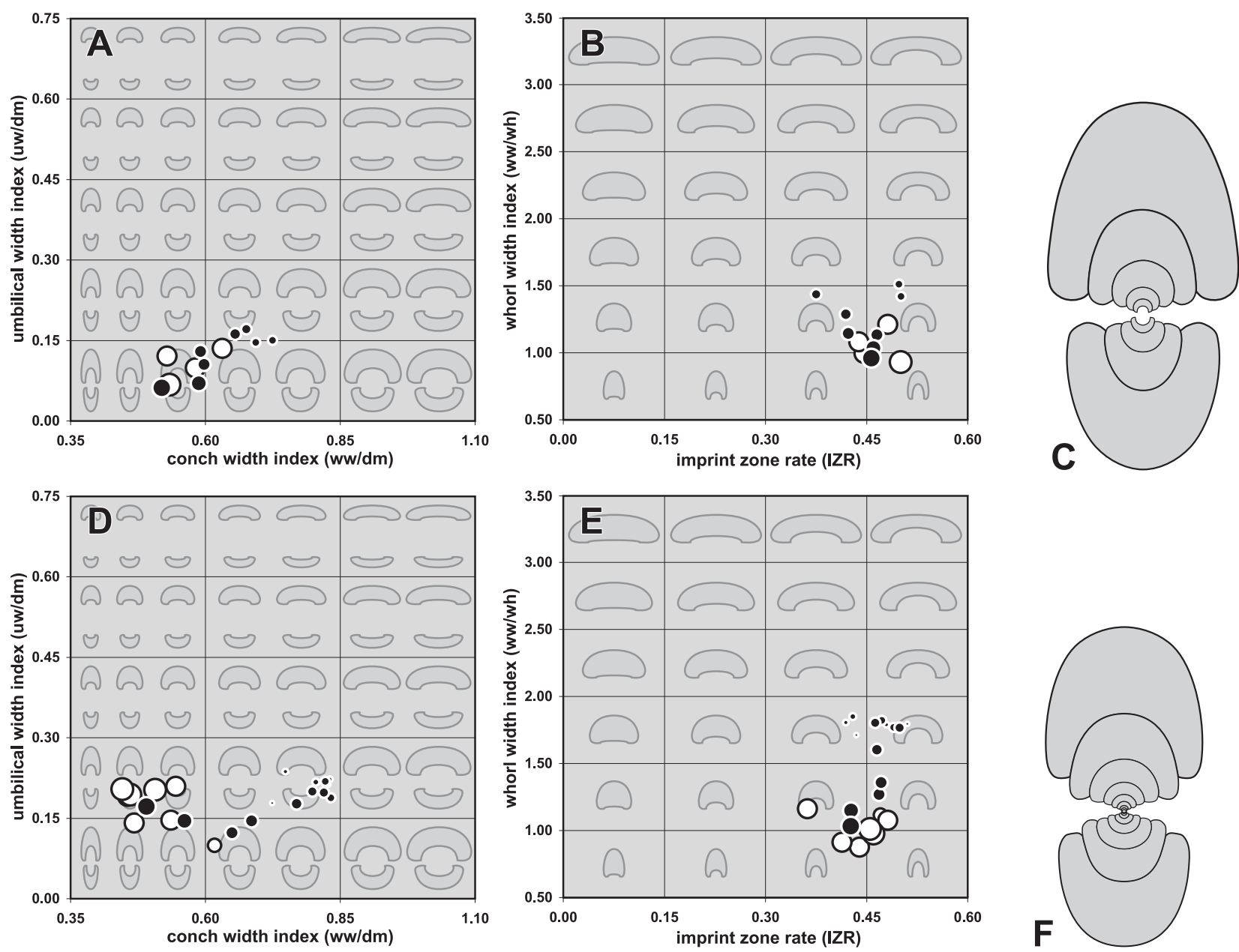

Figure 19. Ontogenetic trajectories of Muensteroceras, exemplified for M. fabrei n. sp. (A-C) from locality TIM-C7 and M. koninckianum Schindewolf, 1951 (D-F) from Pauquys and Furfooz (Belgium). A. Ontogenetic development of the conch width index (ww/dm), umbilical width index (uw/dm). B. Ontogenetic development of the imprint zone rate (IZR), whorl width index (ww/wh). C. Cross section of paratype MB.C.18607.5; $\times 1.0$. D. Ontogenetic development of the conch width index (ww/dm), umbilical width index (uw/dm). E. Ontogenetic development of the imprint zone rate (IZR), whorl width index (ww/wh). F. Cross section of specimen MRH2; $\times 1.0$. [Black dots represent ontogenetic stages of cross sections MB.C.18607.5 and MRH2, white dots represent the reference specimens (Tab. 19 for Muensteroceras fabrei).] 


\section{Muensteroceras fabrei $\mathbf{n}$. sp.}

Figures 20, 21

Derivation of name. After Jean Fabre, in honour of his contribution to Carboniferous geology in Algeria.

Holotype. Specimen MB.C.18607.1, illustrated in Figure 20B.

Type locality and horizon. Sebkha de Timimoun, locality TIM-C7 (14.5 km west-southwest of Timimoun, Algeria); Ammonellipsites-Merocanites Assemblage.

Material. A total of 42 specimens between 38 and $90 \mathrm{~mm}$ conch diameter are available. The maximum conch diameter can be estimated to be up to $120 \mathrm{~mm}$. Most of the specimens are somewhat corroded, but some of them show shell remains.

Diagnosis. Muensteroceras with a subinvolute to involute conch throughout ontogeny, shape thickly discoidal to thinly pachyconic at $10 \mathrm{~mm}$ and thinly to thickly discoidal at $60 \mathrm{~mm} \mathrm{dm}$; umbilical margin rounded in all growth stages; aperture low in early growth stages and high in the adult stage. Shell almost smooth, ornament with very fine biconvex growth lines, course biconvex with very low lateral sinus and deep ventral sinus, direction rectiradiate; usually without shell constrictions. Suture line with very narrow, parallel-sided external lobe and low median saddle; ventrolateral saddle almost symmetric, broadly rounded; adventive lobe V-shaped with rather straight ventral flank and weekly curved dorsal flank.

Table 18. Conch ontogeny (Figs $21 \mathrm{~A}-\mathrm{C}, \mathrm{F}-\mathrm{H}$ ) of Muensteroceras fabrei $\mathrm{n}$. sp.

\begin{tabular}{|c|c|c|c|}
\hline $\mathrm{dm}$ & conch shape & whorl cross section shape & aperture \\
\hline $4 \mathrm{~mm}$ & $\begin{array}{l}\text { thinly pachyconic; subinvolute } \\
(\mathrm{ww} / \mathrm{dm}=0.65-0.70 ; \mathrm{uw} / \mathrm{dm}=0.15-0.20)\end{array}$ & $\begin{array}{l}\text { weakly depressed; very strongly embracing } \\
\text { (ww/wh }=1.30-1.50 ; \mathrm{IZR}=0.45-0.50)\end{array}$ & $\begin{array}{l}\text { low } \\
(\text { WER }=1.65-1.75)\end{array}$ \\
\hline $10 \mathrm{~mm}$ & $\begin{array}{l}\text { thickly discoidal to thinly pachyconic; involute } \\
\text { (ww/dm }=0.55-0.65 ; \text { uw/dm }=0.07-0.15 \text { ) }\end{array}$ & $\begin{array}{l}\text { weakly depressed; strongly embracing } \\
(\mathrm{ww} / \mathrm{wh}=1.00-1.25 ; \mathrm{IZR}=0.40-0.45)\end{array}$ & $\begin{array}{l}\text { high } \\
(\text { WER }=2.00-2.15)\end{array}$ \\
\hline $30 \mathrm{~mm}$ & $\begin{array}{l}\text { thickly discoidal to thinly pachyconic; involute } \\
(\mathrm{ww} / \mathrm{dm}=0.50-0.65 ; \mathrm{uw} / \mathrm{dm}=0.07-0.15 \text { ) }\end{array}$ & $\begin{array}{l}\text { weakly depressed; strongly embracing } \\
(\mathrm{w} w / \mathrm{wh}=1.00-1.25 ; \mathrm{IZR}=0.40-0.45)\end{array}$ & $\begin{array}{l}\text { moderate to high } \\
(\text { WER }=1.95-2.15)\end{array}$ \\
\hline $60 \mathrm{~mm}$ & $\begin{array}{l}\text { thinly to thickly discoidal; involute } \\
(\mathrm{w} w / \mathrm{dm}=0.45-0.55 ; \mathrm{uw} / \mathrm{dm}=0.07-0.12)\end{array}$ & $\begin{array}{l}\text { weakly compressed; very strongly embracing } \\
(\mathrm{w} w / \mathrm{wh}=0.80-1.00 ; \mathrm{IZR}=0.45-0.50)\end{array}$ & $\begin{array}{l}\text { moderate to high } \\
(\text { WER }=1.90-2.10)\end{array}$ \\
\hline
\end{tabular}

Table 19. Conch dimensions (in $\mathrm{mm}$ ) and proportions for reference specimens of Muensteroceras fabrei $\mathrm{n}$. sp.

\begin{tabular}{llllllllllll}
\hline & $\mathrm{dm}$ & ww & wh & uw & ah & ww/dm & ww/wh & uw/dm & WER & IZR \\
\hline paratype MB.C.18607.2 & 70.2 & 37.4 & 40.0 & 4.7 & 20.0 & 0.53 & 0.94 & 0.07 & 1.95 & 0.50 \\
paratype MB.C.18607.9 & 61.2 & 33.4 & 32.4 & 4.8 & 17.2 & 0.55 & 1.03 & 0.08 & 1.94 & 0.47 \\
paratype MB.C.18607.7 & 55.0 & 29.1 & 29.1 & 6.7 & 16.1 & 0.53 & 1.00 & 0.12 & 2.00 & 0.45 & \\
paratype MB.C.18607.3 & 46.9 & 27.3 & 25.1 & 4.7 & 14.1 & 0.58 & 1.09 & 0.10 & 2.04 & 0.44 \\
holotype MB.C.18607.1 & 45.8 & 28.9 & 23.8 & 6.2 & 12.4 & 0.63 & 1.22 & 0.14 & 1.87 & 0.48 \\
\hline
\end{tabular}

Table 20. Suture line proportions (Figs 21D, E) for Muensteroceras fabrei n. sp.

\begin{tabular}{llllllll}
\hline specimen & at dm & EL w/d & EL/NLS & EL/AL & MS h & VLS w/h & remarks \\
\hline paratype MB.C.18607.7 & $43.8 \mathrm{~mm}$ & 0.46 & 0.52 & 0.84 & 0.24 & 0.88 & flanks of E lobe slightly diverging \\
paratype MB.C.18607.8 & c. $40.0 \mathrm{~mm}$ & 0.41 & 0.55 & 0.96 & 0.25 & 0.74 & A lobe with curved dorsal flank \\
\hline
\end{tabular}

Discussion. Muensteroceras fabrei belongs to the few very narrowly umbilicate species with an almost smooth conch within the genus. The lack of constrictions is a character that makes M. fabrei a rather unique species.

M. fabrei differs from the co-occurring Dzhaprakoceras dzhazairense, which has a similar conch shape, in the parallel-sided external lobe that does not show the pouching typical for species of Dzhaprakoceras. The other species of the Dalle à Merocanites from Timimoun have a stouter conch and are thus easily separable from $M$. fabrei.

A similar species is M. koninckianum Schindewolf, 1951 (= Goniatites inconstans de Koninck, 1880), but this species differs in its adult opening of the umbilicus and its subangular ventrolateral shoulder from M. fabrei.

The co-occurring species Trimorphoceras crassilens n. sp. is very similar in the adult stage, and only well-preserved specimens can clearly be separated when the conch ontogeny is not known (Trimorphoceras crassilens possesses, in contrast to $M$. fabrei a widely umbilicate intermediate growth stage). For a detailed comparison, see under that species. 

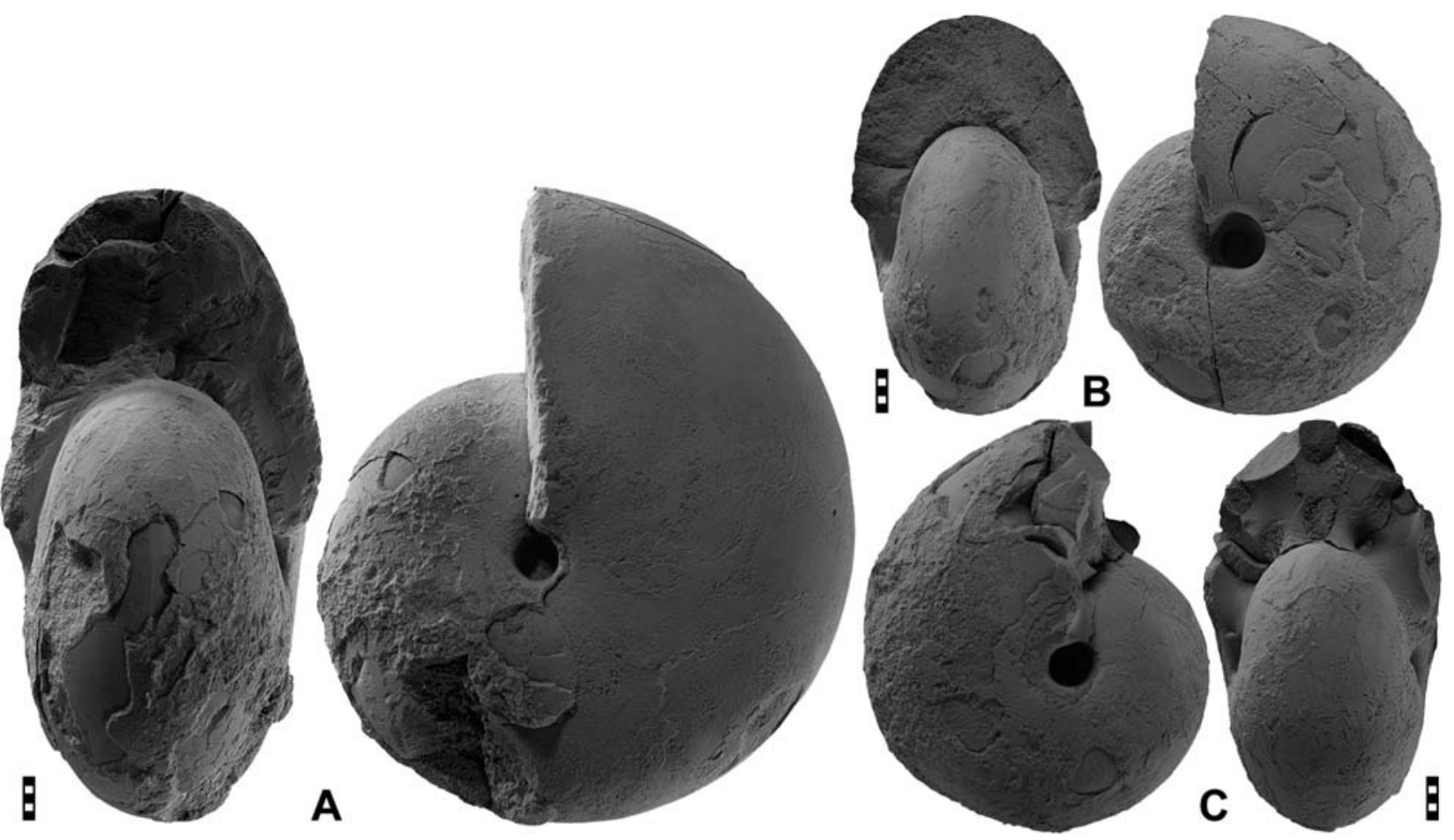

Figure 20. Muensteroceras fabrei n. sp. from locality TIM-C7; all × 1.0. A. Paratype MB.C.18607.2. B. Holotype MB.C.18607.1. C. Paratype MB.C.18607.3.
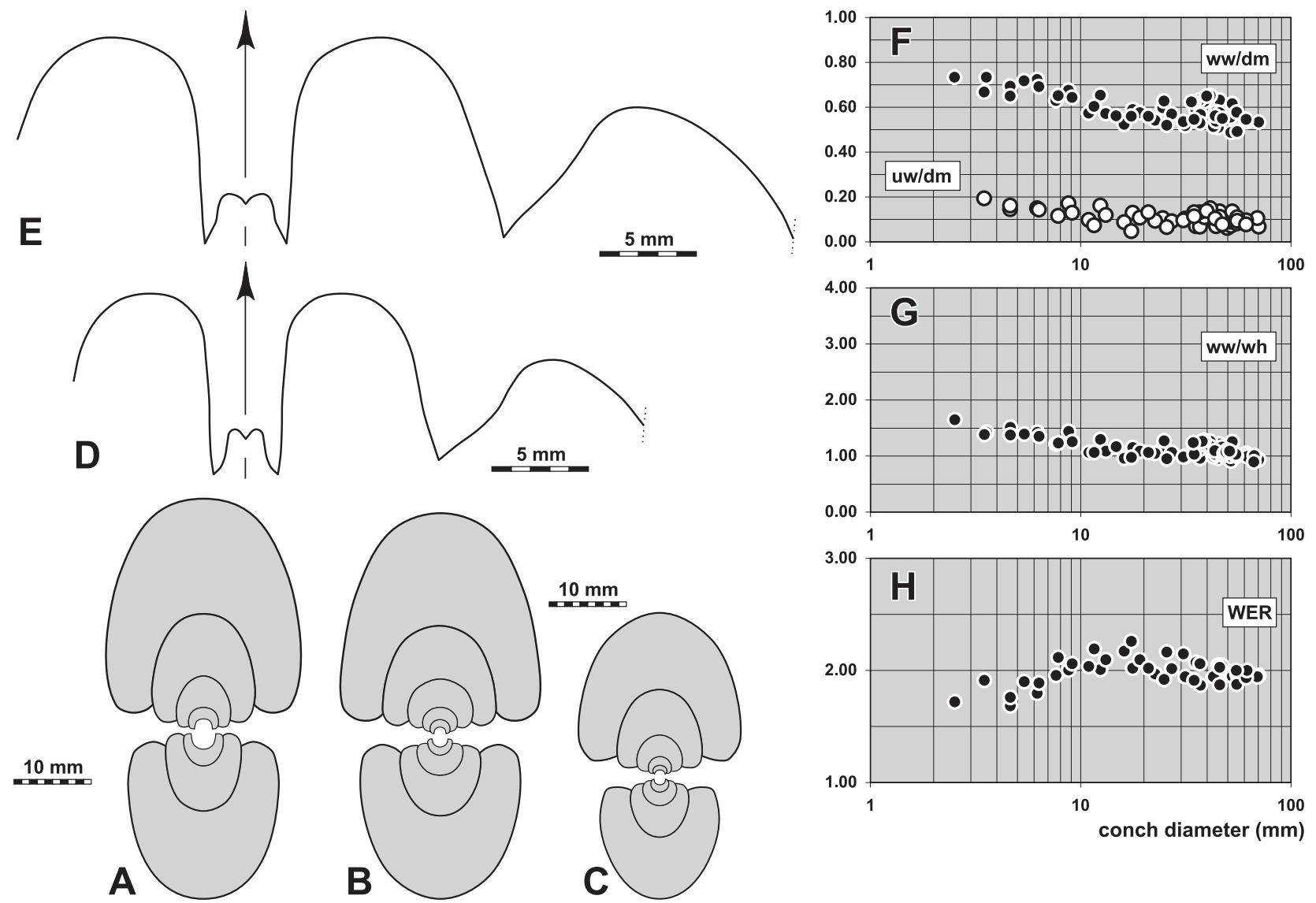

$10 \mathrm{~mm}$
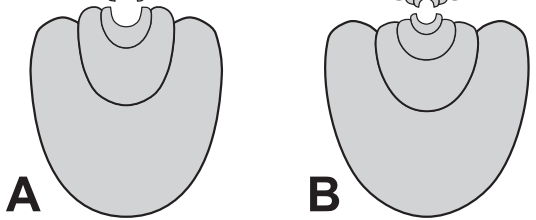

Figure 21. Muensteroceras fabrei n. sp. from locality TIM-C7. A. Cross section of paratype MB.C.18607.4; $\times 1.0$. B. Cross section of paratype MB.C.18607.5; $\times 1.0$. C. Cross section of paratype MB.C.18607.6; $\times 1.0$. D. Suture line of paratype MB.C.18607.8, at $19.3 \mathrm{~mm}$ ww, $16.3 \mathrm{~mm}$ wh; $\times 2.5$. E. Suture line of paratype MB.C. 18607.7 , at $43.8 \mathrm{~mm} \mathrm{dm}, 24.8 \mathrm{~mm}$ ww, $23.5 \mathrm{~mm}$ wh; $\times 2.5$. FH. Ontogenetic development of the conch width index (ww/dm), umbilical width index (uw/dm), whorl width index (ww/wh), and whorl expansion rate (WER) of all available specimens. 
Familiy Rotopericyclidae Korn, Bockwinkel \& Ebbighausen, 2010

For more detailed information about the family, see Korn et al. (2010a).

Family definition. Pericyclaceae with a conch ontogeny that includes an increase of the uw/dm ratio, beginning in the adult stage and progressing onto juveniles. Suture line with parallel-sided or V-shaped external lobe with subparallel flanks.

Included subfamilies.

Rotopericyclinae Korn, Bockwinkel \& Ebbighausen, 2010

Trimorphoceratinae n. subfam.

\section{Subfamiliy Rotopericyclinae Korn, Bockwinkel \& Ebbighausen, 2010}

Subfamily definition. Rotopericyclidae with a conch ontogeny that includes an increase of the uw/dm ratio in the intermediate or adult stage, without significant re-closure of the umbilicus.

Included genera.

Eurites Kusina, 1973

Mouydiria Korn, Bockwinkel \& Ebbighausen, 2010

Rotopericyclus Turner, 1948

Rhnetites Bockwinkel, Korn \& Ebbighausen, 2010

Parahammatocyclus Riley, 1996

\section{Eurites Kusina, 1973}

For a detailed discussion of the genus, see Korn et al. (2010a).

Separation of the new species. The two species of Eurites from the Dalle à Merocanites can easily be separated by their conch ontogeny:

- Eurites commutatus - the umbilicus is closed at a conch diameter of $30-40 \mathrm{~mm}$, when the whorls show increasing overlap onto the preceding volution, reduction of the uw/dm ratio of $0.20-0.35$ at $30 \mathrm{~mm}$ dm to $0.20-0.25$ at $60 \mathrm{~mm} \mathrm{dm}$;

- Eurites pondus - the umbilicus widens almost continuously, reduction of the uw/dm ratio of $0.27-0.38$ at $30 \mathrm{~mm}$ dm to $0.25-0.30$ at $60 \mathrm{~mm}$ $\mathrm{dm}$.

The suture line shows more differences:

- Eurites commutatus - adventive lobe almost symmetric;

- Eurites pondus - adventive lobe asymmetric with rather straight ventral flank and strongly curved dorsal flank.
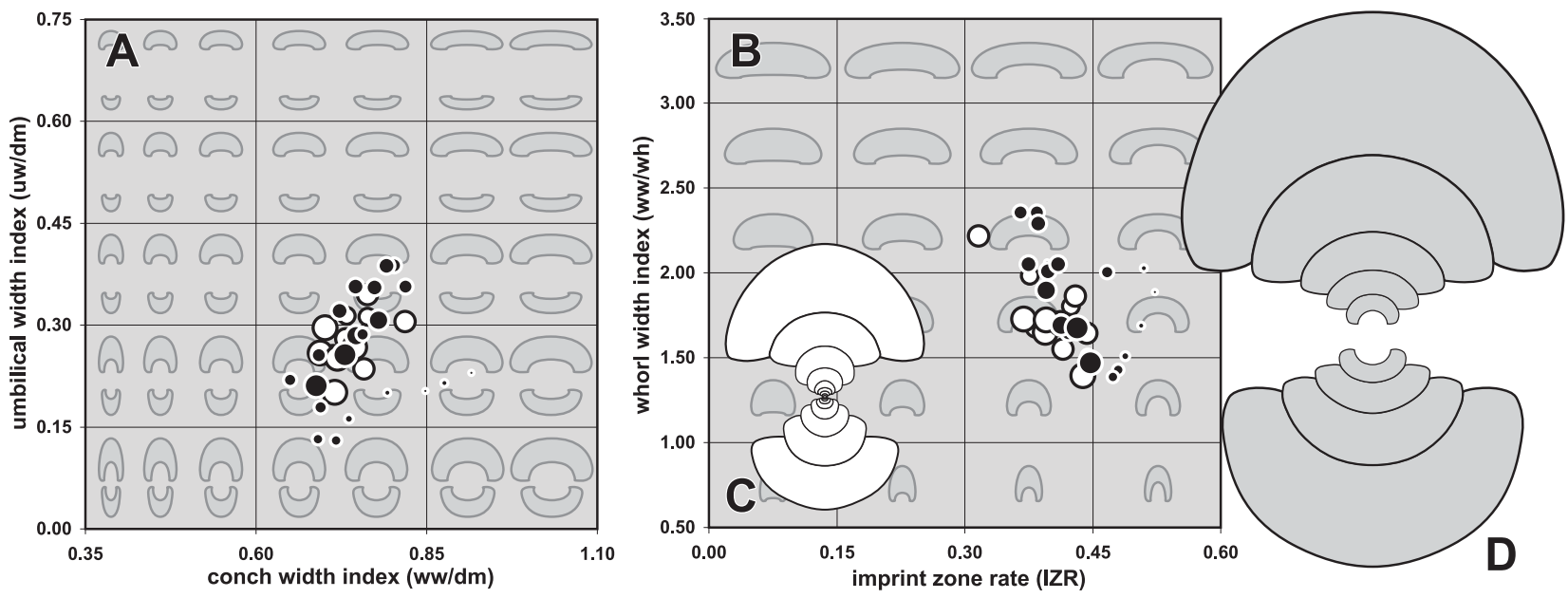

Figure 22. Ontogenetic trajectories of Eurites, exemplified for Eurites commutatus n. sp. from locality TIM-C7. A. Ontogenetic development of the conch width index (ww/dm), umbilical width index (uw/dm). B. Ontogenetic development of the imprint zone rate (IZR), whorl width index (ww/wh). C. Cross section of paratype MB.C.18608.13; ×1.0. D. Cross section of paratype MB.C. $18608.11 ; \times 1.0$. [Black dots represent ontogenetic stages of cross sections MB.C.18608.13 and MB.C.18608.11, white dots represent the reference specimens (Tab. 22).] 
Discussion. The ontogenetic development of the species within the genus Eurites is known in great detail from only the new Algerian material, and hence it is not clear if all the listed species in fact belong to this genus. A species that obviously shows the ontogenetic loop with temporary opening of the umbilicus is E. corpulentissimus (Schindewolf, 1951), which, according to the figures and measurement list provided by Schindewolf (1951), resembles the species from the Dalle à Merocanites.

The type species E. latus Kusina, 1973 from the North Urals possesses obviously a trend towards rapid umbilical opening during intermediate intervals of its ontogeny, but a closure of the umbilicus cannot be seen. It resembles, in this respect, the new species E. pondus.

\section{Eurites commutatus n. sp.}

Figures 23-25

Derivation of name. After Lat. commutatus = changing, because of the ontogenetic changes of conch shape.

Holotype. Specimen MB.C.18608.1, illustrated in Figure 23.

Type locality and horizon. Sebkha de Timimoun, locality TIM-C7 (14.5 km west-southwest of Timimoun, Algeria); Ammonellipsites-Merocanites Assemblage.

Material. The species is represented by well-preserved material; 62 specimens between 19 and 75 mm conch diameter are available. The maximum diameter including the body chamber is estimated to be $120 \mathrm{~mm}$.

Diagnosis. Eurites with a subinvolute conch in juveniles, a subevolute conch at $20 \mathrm{~mm} \mathrm{dm}$, and a subinvolute conch in the adult stage; shape thinly to thickly pachyconic between 20 and $60 \mathrm{~mm} \mathrm{dm}$; umbilical wall flattened, umbilical margin subangular; aperture low to moderate up to $25 \mathrm{~mm} \mathrm{dm}$ and moderate in the adult stage. Shell almost smooth, ornament with very fine biconvex growth lines, course biconvex with very low lateral sinus and deep ventral sinus, direction rectiradiate; shallow constrictions occur irregularly parallel to the growth lines. Suture line with very narrow, parallel-sided external lobe and low median saddle; ventrolateral saddle broadly rounded; adventive lobe V-shaped, almost symmetric.

Table 21. Conch ontogeny (Figs $25 \mathrm{~A}-\mathrm{F}, \mathrm{J}-\mathrm{L}$ ) of Eurites commutatus n. sp.

\begin{tabular}{|c|c|c|c|}
\hline$d m$ & conch shape & whorl cross section shape & aperture \\
\hline $4 \mathrm{~mm}$ & $\begin{array}{l}\text { thinly to thickly pachyconic; subinvolute } \\
(w w / d m=0.70-0.80 ; u w / d m=0.15-0.22)\end{array}$ & $\begin{array}{l}\text { weakly depressed to moderately depressed; } \\
\text { very strongly embracing } \\
(\text { ww/wh }=1.35-1.80 ; \mathrm{IZR}=0.45-0.50)\end{array}$ & $\begin{array}{l}\text { low to moderate } \\
(\mathrm{WER}=1.60-1.90)\end{array}$ \\
\hline $10 \mathrm{~mm}$ & $\begin{array}{l}\text { thinly to thickly pachyconic; subinvolute } \\
\text { (ww/dm }=0.63-0.77 ; \text { uw/dm }=0.20-0.30 \text { ) }\end{array}$ & $\begin{array}{l}\text { moderately depressed; strongly to very strongly embracing } \\
\text { (ww/wh }=1.50-2.00 ; \mathrm{IZR}=0.40-0.50 \text { ) }\end{array}$ & $\begin{array}{l}\text { low to moderate } \\
(\mathrm{WER}=1.55-1.90)\end{array}$ \\
\hline $30 \mathrm{~mm}$ & $\begin{array}{l}\text { thickly pachyconic; subinvolute to subevolute } \\
\text { (ww/dm }=0.70-0.85 ; \mathrm{uw} / \mathrm{dm}=0.20-0.35 \text { ) }\end{array}$ & $\begin{array}{l}\text { moderately to strongly depressed; strongly embracing } \\
\text { (ww/wh }=1.65-2.25 ; \mathrm{IZR}=0.35-0.45 \text { ) }\end{array}$ & $\begin{array}{l}\text { low to moderate } \\
(\mathrm{WER}=1.60-1.85)\end{array}$ \\
\hline $60 \mathrm{~mm}$ & $\begin{array}{l}\text { thinly pachyconic; subinvolute } \\
\text { (ww/dm }=0.60-0.70 ; u w / d m=0.20-0.25 \text { ) }\end{array}$ & $\begin{array}{l}\text { weakly depressed to moderately depressed; strongly } \\
\text { embracing } \\
\text { (ww/wh }=1.40-1.60 ; \text { IZR }=0.35-0.45 \text { ) }\end{array}$ & $\begin{array}{l}\text { moderate } \\
(\mathrm{WER}=1.80-2.00)\end{array}$ \\
\hline
\end{tabular}

Table 22. Conch dimensions (in $\mathrm{mm}$ ) and proportions for reference specimens of Eurites commutatus n. sp.

\begin{tabular}{|c|c|c|c|c|c|c|c|c|c|c|}
\hline & $\mathrm{dm}$ & ww & wh & uw & ah & ww/dm & ww/wh & $\mathrm{uw} / \mathrm{dm}$ & WER & IZR \\
\hline holotype MB.C.18608.1 & 74.8 & 43.5 & 33.6 & 16.5 & 19.4 & 0.58 & 1.29 & 0.22 & 1.82 & 0.42 \\
\hline paratype MB.C.18608.17 & 52.8 & 37.8 & 27.0 & 10.6 & 15.2 & 0.72 & 1.40 & 0.20 & 1.97 & 0.44 \\
\hline paratype MB.C.18608.18 & 42.4 & 29.4 & 17.0 & 11.0 & 10.7 & 0.69 & 1.73 & 0.26 & 1.79 & 0.37 \\
\hline paratype MB.C.18608.4 & 42.2 & 31.4 & 19.0 & 11.3 & 11.5 & 0.74 & 1.65 & 0.27 & 1.89 & 0.39 \\
\hline paratype MB.C.18608.2 & 41.8 & 30.0 & 17.6 & 10.5 & 10.3 & 0.72 & 1.71 & 0.25 & 1.77 & 0.41 \\
\hline paratype MB.C.18608.3 & 41.4 & 29.0 & 16.8 & 12.3 & 10.2 & 0.70 & 1.73 & 0.30 & 1.76 & 0.40 \\
\hline paratype MB.C.18608.5 & 38.4 & 29.1 & 17.3 & 9.0 & 10.6 & 0.76 & 1.69 & 0.24 & 1.92 & 0.38 \\
\hline paratype MB.C.18608.16 & 37.7 & 26.9 & 17.3 & 9.6 & 10.1 & 0.71 & 1.55 & 0.26 & 1.87 & 0.42 \\
\hline paratype MB.C.18608.8 & 30.6 & 23.3 & 10.5 & 10.6 & 7.2 & 0.76 & 2.22 & 0.35 & 1.71 & 0.32 \\
\hline paratype MB.C. 18608.10 & 27.8 & 22.7 & 12.2 & 8.5 & 6.9 & 0.82 & 1.87 & 0.31 & 1.78 & 0.43 \\
\hline paratype MB.C.18608.9 & 27.7 & 20.3 & 12.3 & 7.8 & 6.9 & 0.73 & 1.65 & 0.28 & 1.76 & 0.44 \\
\hline paratype MB.C.18608.6 & 20.2 & 14.8 & 8.2 & 6.4 & 4.7 & 0.73 & 1.81 & 0.31 & 1.70 & 0.42 \\
\hline paratype MB.C.18608.7 & 19.5 & 14.9 & 7.5 & 6.1 & 4.7 & 0.76 & 1.99 & 0.31 & 1.73 & 0.38 \\
\hline
\end{tabular}


Table 23. Suture line proportions (Figs 25G-I) for Eurites commutatus n. sp.

\begin{tabular}{llllllll}
\hline specimen & at dm & EL w/d & EL/VLS & EL/AL & MS h & VLS w/h & remarks \\
\hline paratype MB.C.18608.4 & $39.6 \mathrm{~mm}$ & 0.30 & 0.45 & 0.76 & 0.20 & 0.68 & E lobe slightly pouched \\
paratype MB.C. 18608.14 & c. $39.0 \mathrm{~mm}$ & 0.35 & 0.53 & 0.79 & 0.17 & 0.66 & E lobe parallel-sided \\
paratype MB.C.18608.16 & c. $25.0 \mathrm{~mm}$ & 0.49 & 0.69 & 0.81 & 0.22 & 0.71 & \\
\hline
\end{tabular}

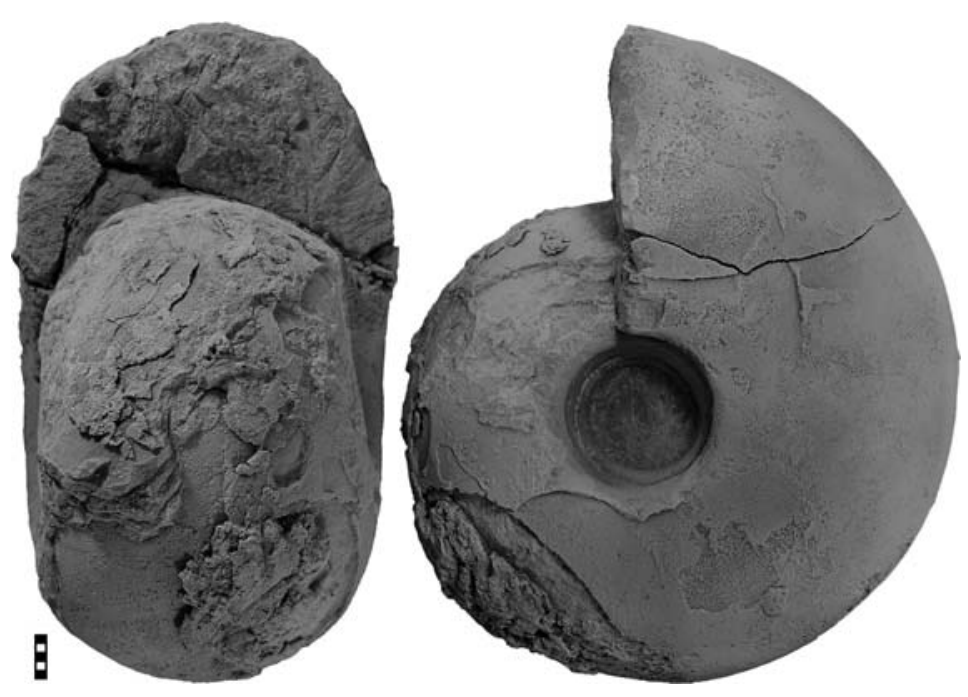

Figure 23. Eurites commutatus n. sp., holotype MB.C.18608.1 from locality TIM-C7; $\times 1.0$.
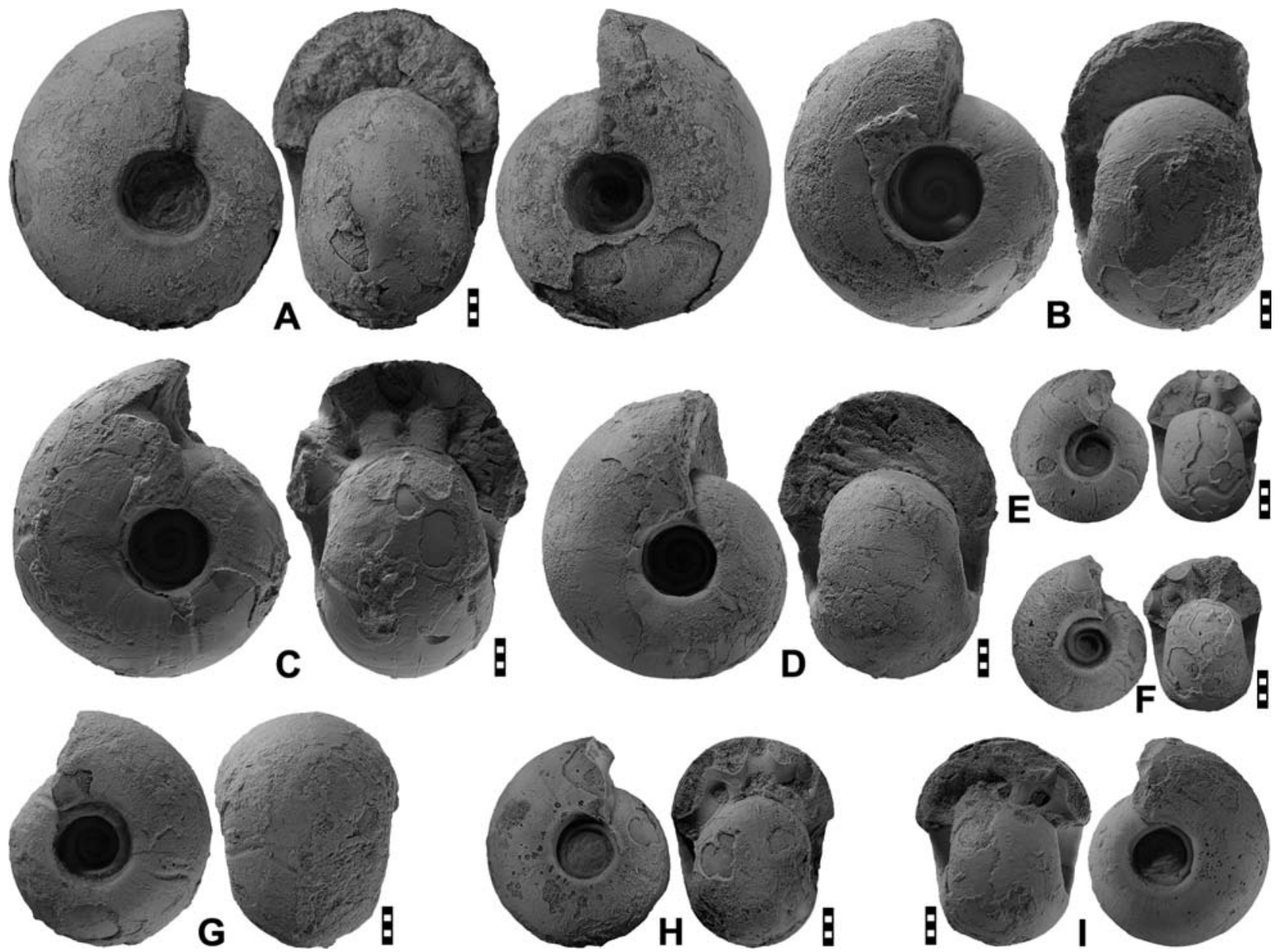

Figure 24. Eurites commutatus n. sp. from locality TIM-C7; all ×1.0. A. Paratype MB.C.18608.2. B. Paratype MB.C.18608.3. C. Paratype MB.C.18608.4. D. Paratype MB.C.18608.5. E. Paratype MB.C.18608.6. F. Paratype MB.C.18608.7. G. Paratype MB.C.18608.8. H. Paratype MB.C.18608.9. I. Paratype MB.C.18608.10. 
Discussion. Eurites commutatus closely resembles the co-occurring species E. pondus, and small specimens up to a conch diameter of 20-25 $\mathrm{mm}$ are probably not separable with certainty. In E. commutatus, the umbilicus is being closed at a conch diameter of $30-40 \mathrm{~mm}$, when the whorls show increasing overlap onto the preceding volution, whereas in E. pondus the umbilicus widens almost continuously. The suture line of E. commutatus has a more symmetric, V-shaped adventive lobe, which is strikingly asymmetric in E. pondus.

The type species of the genus, E. latus Kusina, 1973, has a slightly wider conch (ww/dm $=0.78$ at $48 \mathrm{~mm} \mathrm{dm}$ ) than E. commutatus (ww/dm about 0.70 at the same diameter). E. latus has a continuously opening umbilicus. E. corpulentissimus (Schindewolf, 1951) is also stouter (ww/dm $=0.78$ at $52 \mathrm{~mm} \mathrm{dm}$ ) than E. commutatus and has a strikingly asymmetric adventive lobe.

The conch ontogeny of the Irish species E. corpulentus (Crick, 1899) and E. ellipsoidalis (Crick, 1899) is unknown, and hence these two species are difficult to compare with the Algerian material. The two Irish species may be conspecific; they possess a conch that is not as stout as E. commutatus $(\mathrm{ww} / \mathrm{dm}=0.61$ at $68 \mathrm{~mm} \mathrm{dm}$ ) but has a wider umbilicus (uw/dm $=0.27$ ).

Juvenile conchs of E. commutatus have some superficial similarities with the co-occurring Hammatocyclus pollex, but can easily be distinguished by the course of constrictions (concavo-convex with deep ventral sinus in E. commutatus, almost linear in H. pollex).

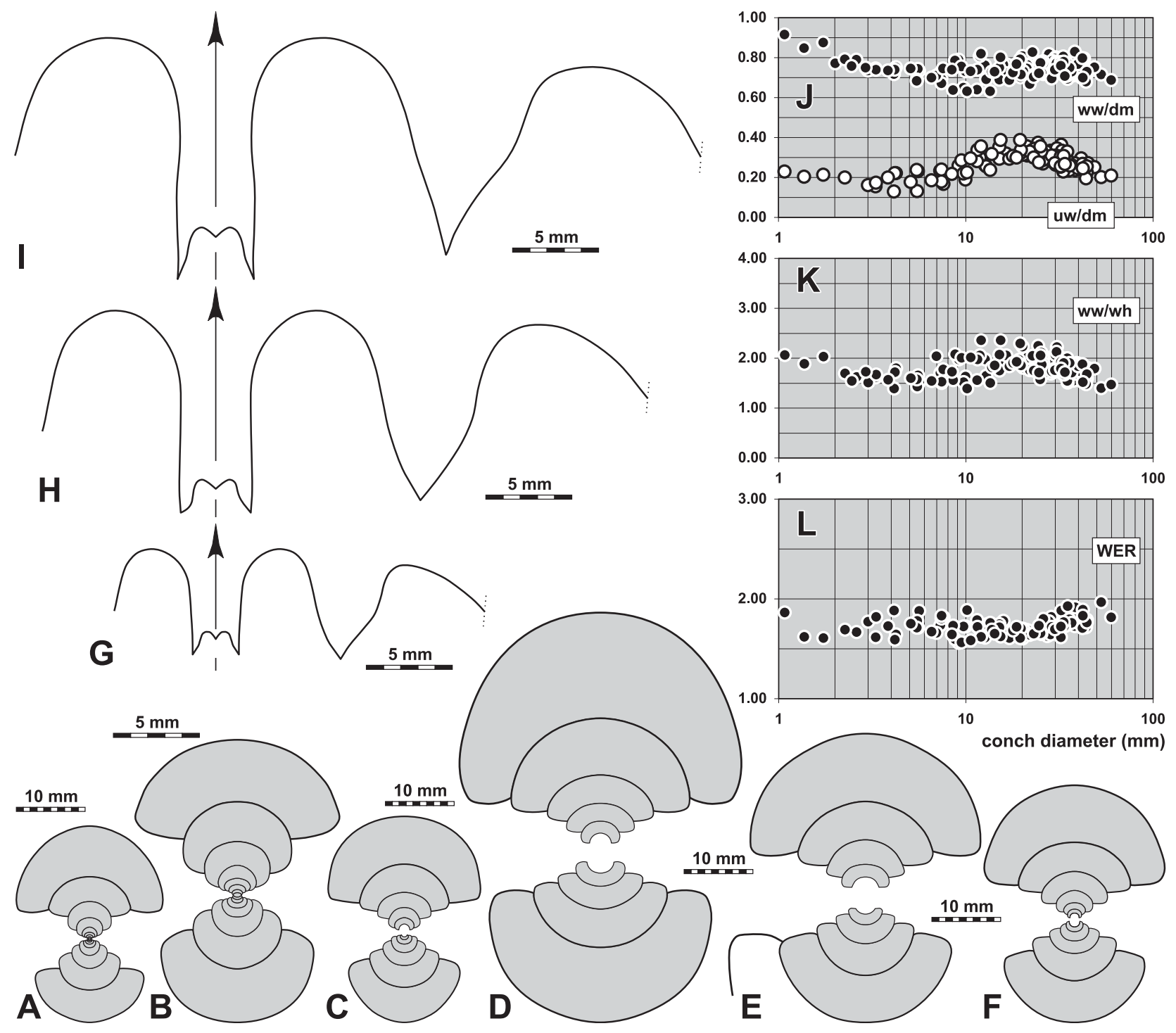

Figure 25. Eurites commutatus n. sp. from locality TIM-C7. A. Cross section of paratype MB.C.18608.11; $\times 1.0$. B. Cross section (inner whorls) of the same specimen; $\times 2.5$. C. Cross section of paratype MB.C.18608.12; $\times 1.0$. D. Cross section of paratype MB.C.18608.13; $\times 1.0$. E. Cross section of paratype MB.C. $18608.14 ; \times 1.0$. F. Cross section of paratype MB.C.18608.15; × 1.0. G. Suture line of paratype MB.C.18608.16, at $19.3 \mathrm{~mm} \mathrm{ww}, 9.0 \mathrm{~mm}$ wh; $\times 2.5$. H. Suture line of paratype MB.C.18608.14, at $31.2 \mathrm{~mm}$ ww, $17.5 \mathrm{~mm}$ wh; $\times 2.5$. I. Suture line of paratype MB.C.18608.4, at $39.6 \mathrm{~mm} \mathrm{dm}, 31.0 \mathrm{~mm} w w, 19.3 \mathrm{~mm}$ wh; $\times 2.5 . \mathbf{J}-$ L. Ontogenetic development of the conch width index (ww/dm), umbilical width index (uw/dm), whorl width index (ww/wh), and whorl expansion rate (WER) of all available specimens. 


\section{Eurites pondus n. sp.}

Figures 26, 27

Derivation of name. After Lat. pondus = weight, because of the voluminous conch shape.

Holotype. Specimen MB.C.18609.1, illustrated in Figure 26A

Type locality and horizon. Sebkha de Timimoun, locality TIM-C7 (14.5 km west-southwest of Timimoun, Algeria); Ammonellipsites-Merocanites Assemblage.

Material. Well-preserved material is available; 51 specimens between 20 and $84 \mathrm{~mm}$ conch diameter were studied. Paratype MB.C.18609.11 is a fully septate specimen with a conch diameter of $84 \mathrm{~mm}$; the size of the phragmocone suggests that the maximum diameter with body chamber was about $150 \mathrm{~mm}$. Most of the specimens are phragmocones with few shell remains.

Diagnosis. Eurites with a subinvolute conch in juveniles and a subinvolute to subevolute conch between 20 and $60 \mathrm{~mm} \mathrm{dm}$; shape thinly to thickly pachyconic at $10 \mathrm{~mm} \mathrm{dm}$ and becoming globular during ontogeny; umbilical wall flattened, umbilical margin subangular; aperture low or moderately high throughout ontogeny. Shell almost smooth with very fine growth lines, course concavo-convex with very shallow lateral sinus, low ventrolateral projection, and moderately deep, subangular ventral sinus, direction rectiradiate. Suture line with very narrow, parallelsided external lobe; median saddle very low or low; ventrolateral saddle broadly rounded and almost symmetric; adventive lobe V-shaped, asymmetric with rather straight ventral flank and strongly curved dorsal flank.

Table 24. Conch ontogeny (Figs $27 \mathrm{~A}-\mathrm{F}, \mathrm{J}-\mathrm{L}$ ) of Eurites pondus n. sp.

\begin{tabular}{|c|c|c|c|}
\hline $\mathrm{dm}$ & conch shape & whorl cross section shape & aperture \\
\hline $4 \mathrm{~mm}$ & $\begin{array}{l}\text { thinly to thickly pachyconic; subinvolute } \\
(\mathrm{ww} / \mathrm{dm}=0.70-0.80 ; \mathrm{uw} / \mathrm{dm}=0.15-0.22)\end{array}$ & $\begin{array}{l}\text { moderately depressed; very strongly embracing } \\
(\mathrm{ww} / \mathrm{wh}=1.50-1.80 ; \mathrm{IZR}=0.45-0.50)\end{array}$ & $\begin{array}{l}\text { low } \\
(\text { WER }=1.60-1.75)\end{array}$ \\
\hline $10 \mathrm{~mm}$ & $\begin{array}{l}\text { thinly to thickly pachyconic; subinvolute to } \\
\text { subevolute (ww/dm }=0.68-0.77 ; \mathrm{uw} / \mathrm{dm}=0.25-0.32 \text { ) }\end{array}$ & $\begin{array}{l}\text { moderately depressed; strongly to very strongly } \\
\text { embracing ( } w w / w h=1.60-2.00 ; \mathrm{ZR}=0.40-0.50 \text { ) }\end{array}$ & $\begin{array}{l}\text { low } \\
(\text { WER }=1.60-1.75)\end{array}$ \\
\hline $30 \mathrm{~mm}$ & $\begin{array}{l}\text { thickly pachyconic; subinvolute to subevolute } \\
(\mathrm{ww} / \mathrm{dm}=0.75-0.85 ; \mathrm{uw} / \mathrm{dm}=0.27-0.38)\end{array}$ & $\begin{array}{l}\text { moderately to strongly depressed; strongly embracing } \\
\text { ( } w w / w h=1.85-2.25 ; \mathrm{IZR}=0.35-0.45)\end{array}$ & $\begin{array}{l}\text { low } \\
(\mathrm{WER}=1.60-1.75)\end{array}$ \\
\hline $60 \mathrm{~mm}$ & $\begin{array}{l}\text { thickly pachyconic to globular; subinvolute } \\
(\mathrm{ww} / \mathrm{dm}=0.80-0.90 ; \mathrm{uw} / \mathrm{dm}=0.25-0.30)\end{array}$ & $\begin{array}{l}\text { moderately depressed; very strongly embracing } \\
(\mathrm{ww} / \mathrm{wh}=1.75-1.90 ; \mathrm{IZR}=0.45-0.50)\end{array}$ & $\begin{array}{l}\text { low to moderate } \\
(\mathrm{WER}=1.60-1.85)\end{array}$ \\
\hline
\end{tabular}
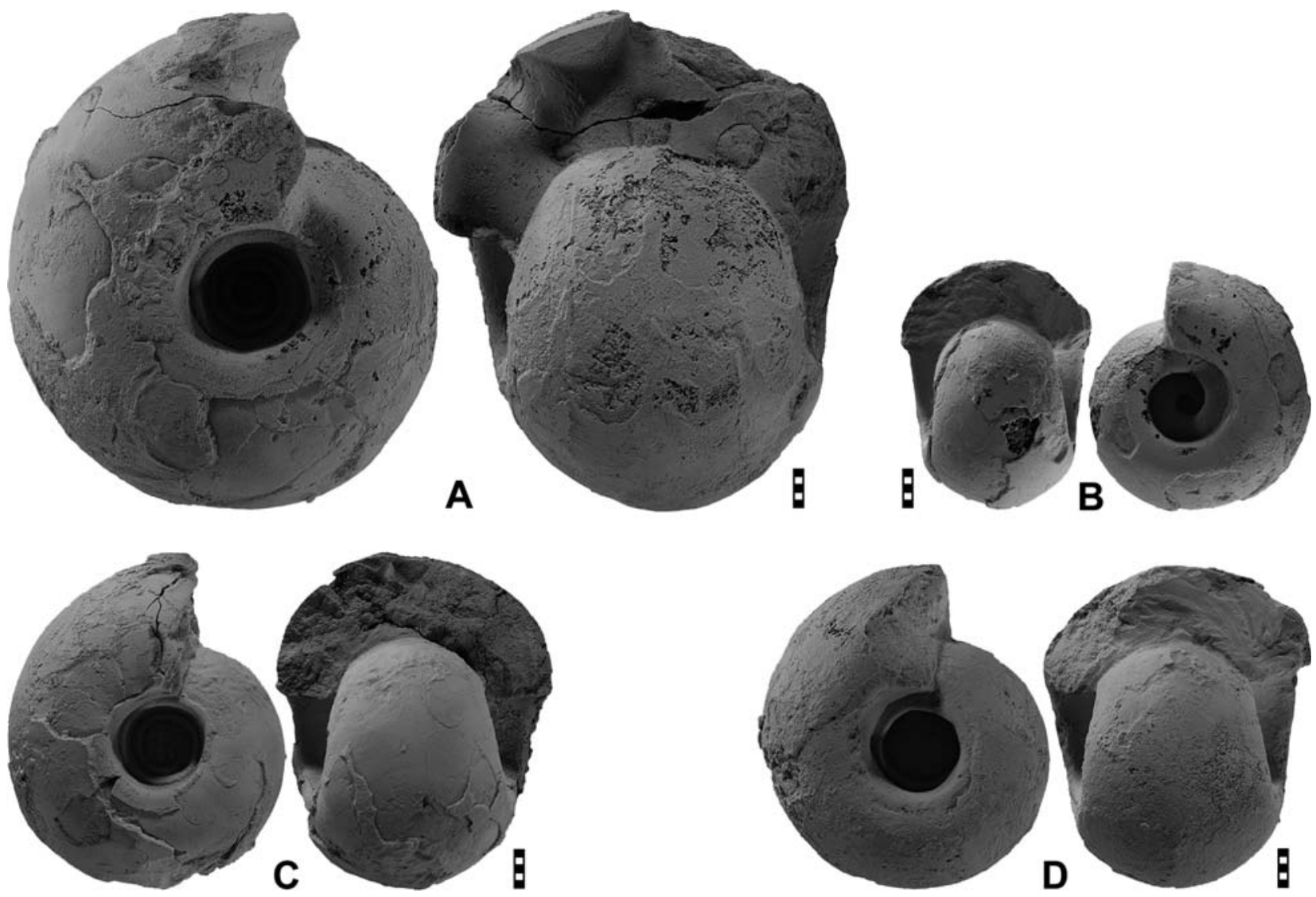

Figure 26. Eurites pondus n. sp. from locality TIM-C7; all $\times 1.0$. A. Holotype MB.C.18609.1. B. Paratype MB.C.18609.2. C. Paratype MB.C.18609.3. D. Paratype MB.C.18609.4. 
Table 25. Conch dimensions (in $\mathrm{mm}$ ) and proportions for reference specimens of Eurites pondus n. sp.

\begin{tabular}{lcccccccccc}
\hline & $\mathrm{dm}$ & ww & wh & uw & ah & ww/dm & ww/wh & uw/dm & WER & IZR \\
\hline paratype MB.C.18609.11 & 83.3 & 71.9 & 39.0 & 22.6 & 20.7 & 0.86 & 1.84 & 0.27 & 1.77 & 0.47 \\
holotype MB.C.18609.1 & 60.8 & 52.4 & 29.0 & 15.1 & 15.6 & 0.86 & 1.81 & 0.25 & 1.81 & 0.46 \\
paratype MB.C.18609.3 & 43.3 & 35.3 & 18.7 & 11.1 & 11.3 & 0.82 & 1.89 & 0.26 & 1.83 & 0.40 \\
paratype MB.C.18609.4 & 41.5 & 33.7 & 16.4 & 13.8 & 10.0 & 0.81 & 2.06 & 0.33 & 1.73 & 0.39 \\
paratype MB.C.18609.12 & 39.6 & 30.6 & 15.8 & 11.1 & 9.9 & 0.77 & 1.94 & 0.28 & 1.77 & 0.37 \\
paratype MB.C.18609.10 & 39.2 & 30.5 & 15.0 & 11.0 & 9.8 & 0.78 & 2.03 & 0.28 & 1.78 & 0.35 \\
paratype MB.C.18609.13 & 37.0 & 29.3 & 14.5 & 10.4 & 8.7 & 0.79 & 2.02 & 0.28 & 1.71 & 0.40 \\
paratype MB.C.18609.2 & 32.2 & 24.3 & 10.8 & 11.2 & 7.5 & 0.75 & 2.25 & 0.35 & 1.69 & 0.31 \\
paratype MB.C.18609.14 & 27.3 & 21.6 & 10.2 & 9.0 & 6.0 & 0.79 & 2.13 & 0.33 & 1.64 & 0.41 \\
\hline
\end{tabular}
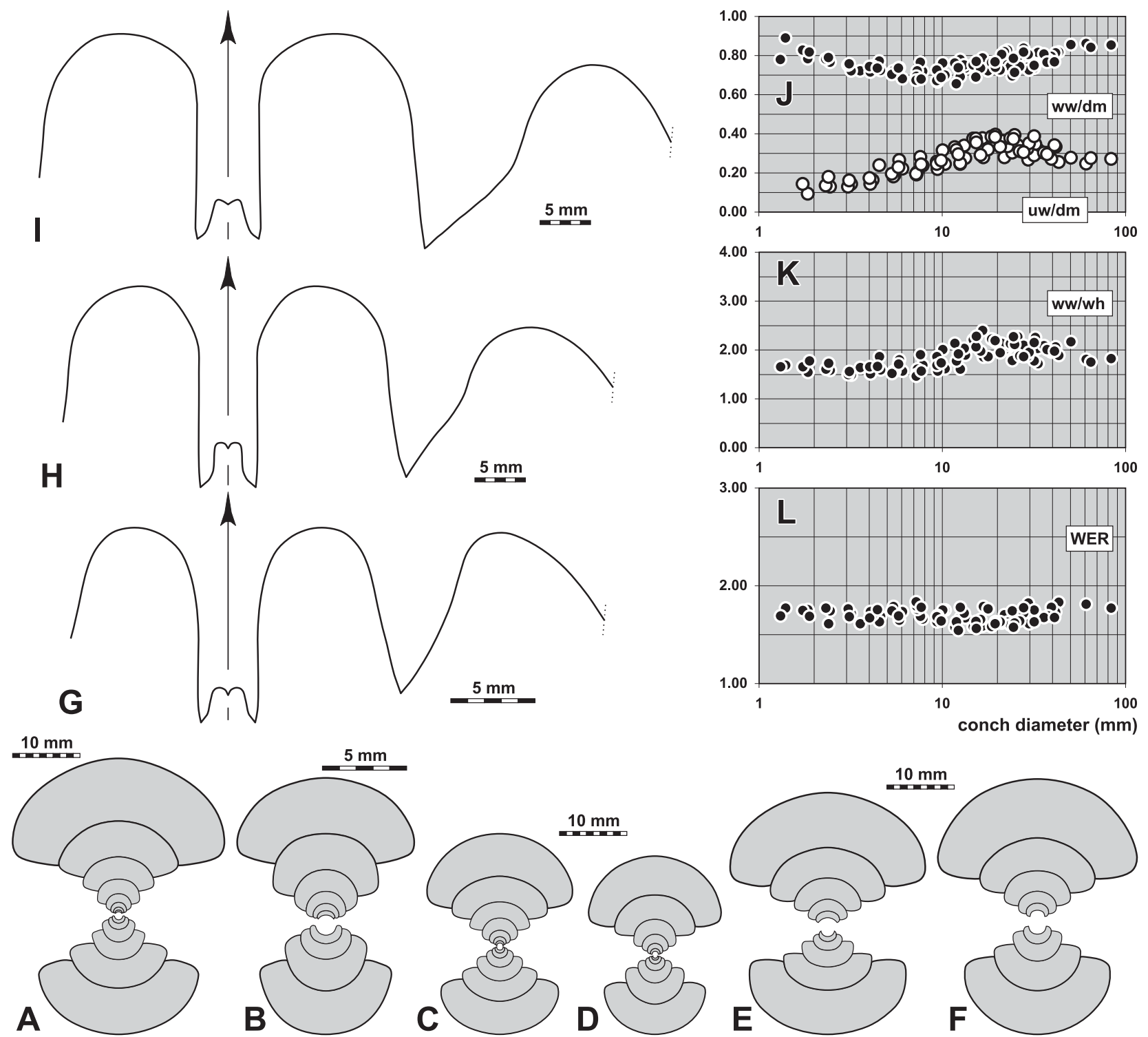

Figure 27. Eurites pondus n. sp. from locality TIM-C7. A. Cross section of paratype MB.C.18609.5; $\times 1.0$. B. Cross section (inner whorls) of the same specimen; $\times 2.5$. C. Cross section of paratype MB.C.18609.6; $\times 1.0$. D. Cross section of paratype MB.C.18609.7; $\times 1.0$. E. Cross section of paratype MB.C.18609.8; $\times 1.0$. F. Cross section of paratype MB.C.18609.9; $\times 1.0$. G. Suture line of paratype MB.C.18609.10, at $33.5 \mathrm{~mm} \mathrm{dm}, 26.1 \mathrm{~mm} \mathrm{ww}, 14.4 \mathrm{~mm} \mathrm{wh;} \times 2.5$. H. Suture line of holotype MB.C.18609.1, at $59.4 \mathrm{~mm} \mathrm{dm}, 48.5 \mathrm{~mm} \mathrm{ww}, 24.8 \mathrm{~mm} \mathrm{wh;} \times 1.5$. I. Suture line of paratype MB.C.18609.11, at $67.0 \mathrm{~mm} \mathrm{dm}$, $57.0 \mathrm{~mm}$ ww, $27.0 \mathrm{~mm}$ wh; $\times 1.5$. J-L. Ontogenetic development of the conch width index (ww/dm), umbilical width index (uw/ $\mathrm{dm})$, whorl width index (ww/wh), and whorl expansion rate (WER) of all available specimens. 
Table 26. Suture line proportions (Figs $27 \mathrm{G}-\mathrm{I}$ ) for Eurites pondus n. sp.

\begin{tabular}{llllllll}
\hline specimen & at dm & EL w/d & EL/VLS & EL/AL & MS h & VLS w/h & remarks \\
\hline paratype MB.C.18609.11 & $67.0 \mathrm{~mm}$ & 0.30 & 0.41 & 0.57 & 0.19 & 0.75 & A lobe strongly asymmetric \\
holotype MB.C.18609.1 & $59.4 \mathrm{~mm}$ & 0.29 & 0.44 & 0.73 & 0.21 & 0.66 & A lobe almost symmetric \\
paratype MB.C.18609.10 & $33.5 \mathrm{~mm}$ & 0.31 & 0.48 & 0.87 & 0.18 & 0.64 & \\
\hline
\end{tabular}

Discussion. The co-occurring species Eurites commutatus closely resembles E. pondus, but the two species can be separated by the development of the umbilicus and the adventive lobe. In E. commutatus, the umbilicus is being closed at a conch diameter of $30-40 \mathrm{~mm}$, where the whorls show increasing overlap onto the preceding volution, whereas in E. pondus the umbilicus widens almost continuously. The suture line of E. commutatus has a more symmetric, V-shaped adventive lobe, which is strikingly asymmetric in E. pondus.

The type species of the genus, E. latus Kusina, 1973, has a slightly narrower conch $(\mathrm{ww} / \mathrm{dm}=0.78 \mathrm{at} 48 \mathrm{~mm} \mathrm{dm}$ ) than E. pondus (ww/dm about 0.85 at the same diameter). E. latus has a much narrower ventrolateral saddle than E. pondus. E. corpulentissimus (Schindewolf, 1951) is also more slender (ww/dm $=0.78$ at $52 \mathrm{~mm} \mathrm{dm}$ ) but resembles E. commutatus in the strikingly asymmetric adventive lobe. E. corpulentissimus differs particularly in the much narrower umbilicus (uw $/ \mathrm{dm}=0.16$ at $17 \mathrm{~mm} \mathrm{dm}$ ) from $E$. pondus (uw $/ \mathrm{dm}=0.28-0.38$ ) at the same diameter.

E. corpulentus (Crick, 1899) and E. ellipsoidalis (Crick, 1899) are very similar in the opening rate of the umbilicus, but differ in the more slender conch $(\mathrm{ww} / \mathrm{dm}=0.61$ at $68 \mathrm{~mm} \mathrm{dm}$ ) from E. pondus.

\section{Subfamiliy Trimorphoceratinae n. subfam.}

Subfamily definition. Rotopericyclidae with a conch ontogeny that includes an increase of the uw/dm ratio in the intermediate or adult stage and a subsequent re-closure of the umbilicus.

Included genera.
Trimorphoceras n. gen.
Obturgites n. gen.

Discussion. The members of the subfamily differ from the subfamily Rotopericyclinae in the adult stage with reclosure of the umbilicus.

\section{Trimorphoceras n. gen.}

Derivation of name. After Greek tri = three and morphos = shape, because of the three principal postembryonic growth stages of conch morphology.

Type species. Trimorphoceras absolutum n. sp.

Genus definition. Muensteroceratinae with significant ontogenetic changes in the conch geometry; shape pachyconic in juvenile and preadult conchs and rapid ontogenetic development towards a discoidal adult conch; conch involute or subinvolute in juveniles and the adult stage, intermediate stage with striking temporary opening of the umbilicus (Fig. 28); flanks converge towards the rounded venter; aperture low or moderately high in the juvenile and preadult stage, becoming rapidly higher in the adult stage; umbilical wall often flattened, umbilical margin narrowly rounded or subangular. Ornament with very fine, concavo-convex rectiradiate growth lines with subangular ventrolateral projection and rather deep ventral sinus; often with shell constrictions parallel to the growth lines. Suture line with very narrow, parallel-sided external lobe and low median saddle; ventrolateral saddle broadly rounded; adventive lobe V-shaped, symmetric.

Included species.

absolutum: Trimorphoceras absolutum n. sp.; Gourara, Algeria.

azzelmattiense: Trimorphoceras azzelmattiense Korn, Ebbighausen \& Bockwinkel, 2010; Ahnet, Algeria.

crassilens: Trimorphoceras crassilens n. sp.; Gourara, Algeria.

molestum: Trimorphoceras molestum n. sp.; Gourara, Algeria.

sphaeroidale: Goniatites sphaeroidalis M'Coy, 1844: 15. Ireland.

teguentourense: Trimorphoceras teguentourense Korn, Ebbighausen \& Bockwinkel, 2010; Ahnet, Algeria.

? crassum: Glyphioceras (Muensteroceras) crassum Foord, 1903, p. 193; Ireland.

Separation of the new species. The three species of Trimorphoceras from the Dalle à Merocanites can be separated by their conch shape at 30 and $60 \mathrm{~mm} \mathrm{dm:}$

- Trimorphoceras crassilens - at $30 \mathrm{~mm} \mathrm{dm:} \mathrm{conch} \mathrm{thickly} \mathrm{discoidal} \mathrm{to} \mathrm{thinly} \mathrm{pachyconic;} \mathrm{involute} \mathrm{to} \mathrm{subinvolute}(\mathrm{ww} / \mathrm{dm}=0.55-0.65$; uw/ $\mathrm{dm}=0.10-0.20)$; at $60 \mathrm{~mm} \mathrm{dm}$ : conch thinly to thickly discoidal; involute (ww/ $\mathrm{dm}=0.45-0.50 ; \mathrm{uw} / \mathrm{dm}=0.05-0.10)$;

- Trimorphoceras absolutum - at $30 \mathrm{~mm} \mathrm{dm}$ : conch thinly pachyconic; subinvolute $(\mathrm{ww} / \mathrm{dm}=0.60-0.70 ; \mathrm{uw} / \mathrm{dm}=0.20-0.30)$; at $60 \mathrm{~mm} \mathrm{dm}$ : conch thickly discoidal; involute (ww/dm $=0.50-0.60 ; \mathrm{uw} / \mathrm{dm}=0.10-0.15)$;

- Trimorphoceras molestum - at $30 \mathrm{~mm} \mathrm{dm}$ : thickly pachyconic; subinvolute (ww/dm $=0.70-0.85 ; \mathrm{uw} / \mathrm{dm}=0.20-0.30$ ); at $60 \mathrm{~mm} \mathrm{dm}$ : conch thickly discoidal; subinvolute $(\mathrm{ww} / \mathrm{dm}=0.55-0.60 ; \mathrm{uw} / \mathrm{dm}=0.15-0.20)$. 

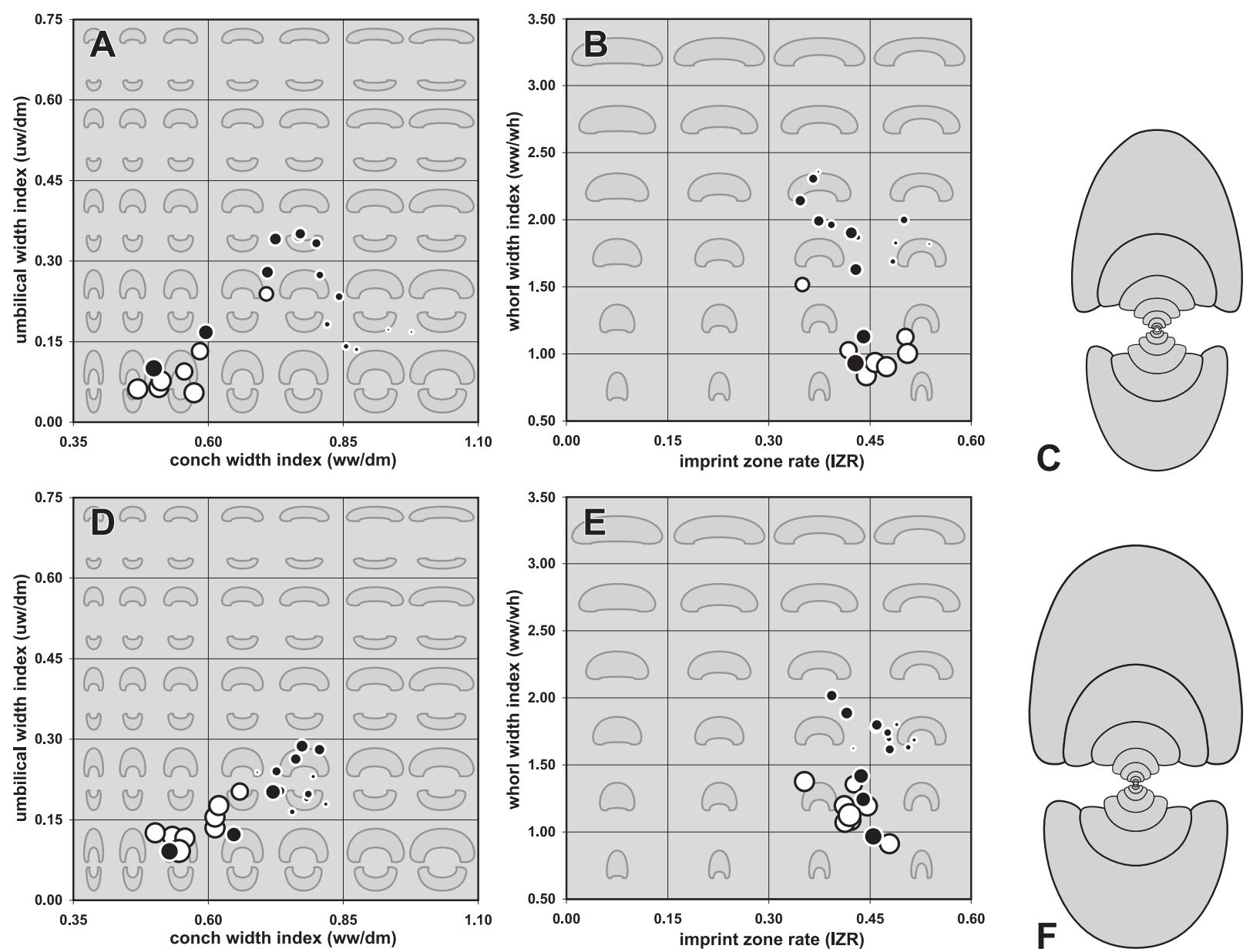

Figure 28. Ontogenetic trajectories of Trimorphoceras, exemplified for Trimorphoceras crassilens n. sp. (A-C) and Trimorphoceras absolutum n. sp. (D-F) from locality TIM-C7. A. Ontogenetic development of the conch width index (ww/dm), umbilical width index (uw/dm). B. Ontogenetic development of the imprint zone rate (IZR), whorl width index (ww/wh). C. Cross section of paratype MB.C.18610.7; $\times 1.0$. D. Ontogenetic development of the conch width index (ww/dm), umbilical width index (uw/ $\mathrm{dm}$ ). E. Ontogenetic development of the imprint zone rate (IZR), whorl width index (ww/wh). F. Cross section of paratype MB.C.18611.6; $\times 1.0$. [Black dots represent ontogenetic stages of cross section MB.C.18610.7 and MB.C.18611.6, white dots represent the reference specimens (Tab. 28, 31).]

Discussion. It is difficult to tell if other already known Late Tournaisian muensteroceratid species belong to Trimorphoceras, because the conch ontogeny is only known from the new species. At least "Goniatites sphaeroidalis Mc Coy, 1844" can be assigned to the new genus with some certainty because of the striking deceleration of whorl width increase in the last volution of that species.

\section{Trimorphoceras crassilens n. sp.}

Figures 29, 30

Derivation of name. After Lat. crassus = thick and lens = lentil, because of the thickly lenticular conch shape.

Holotype. Specimen MB.C.18610.1, illustrated in Figure 29D.

Type locality and horizon. Sebkha de Timimoun, locality TIM-C7 (14.5 km west-southwest of Timimoun, Algeria); Ammonellipsites-Merocanites Assemblage.

Material. A total of 53 specimens between 20 and $70 \mathrm{~mm}$ conch diameter were studied. The largest of these, paratype MB.C.18610.11, is still fully septate, suggesting that the total diameter with body chamber was at least $130 \mathrm{~mm}$.

Diagnosis. Trimorphoceras with a thickly pachyconic conch between 2 and $15 \mathrm{~mm} \mathrm{dm}$, thereafter rapid lateral compression towards a thinly discoidal conch at $70 \mathrm{~mm} \mathrm{dm}$; juvenile stage subinvolute, growth interval between 8 and $15 \mathrm{~mm} \mathrm{dm}$ subevolute, rapid closure of the umbilicus in the adult stage by very strong whorl overlap onto the previous whorl; umbilical margin rounded in juveniles, becoming subangular at $10 \mathrm{~mm}$ $\mathrm{dm}$; umbilical wall flat in intermediate growth stages; aperture low in juveniles and rapidly becoming high in growth stages above $30 \mathrm{~mm} \mathrm{dm}$. Ornament with very fine growth lines; course concavo-convex with very shallow lateral sinus and deep ventral sinus, direction rectiradiate; small specimens with shell constrictions parallel to the growth lines, specimens larger than $30 \mathrm{~mm} \mathrm{dm}$ without constrictions on shell and steinkern. Suture line with very narrow, parallel-sided external lobe and very low or low median saddle; ventrolateral saddle broadly rounded and almost symmetric; adventive lobe V-shaped, slightly asymmetric. 
Table 27. Conch ontogeny (Figs 30A-G, L-N) of Trimorphoceras crassilens $\mathrm{n}$. sp.

\begin{tabular}{|c|c|c|c|}
\hline $\mathrm{dm}$ & conch shape & whorl cross section shape & aperture \\
\hline $4 \mathrm{~mm}$ & $\begin{array}{l}\text { thickly pachyconic; subinvolute } \\
(\mathrm{w} w / \mathrm{dm}=0.75-0.85 ; \mathrm{w} / \mathrm{dm}=0.20-0.30)\end{array}$ & $\begin{array}{l}\text { moderately depressed; very strongly embracing } \\
(\mathrm{ww} / \mathrm{wh}=1.60-2.00 ; \mathrm{IZR}=0.45-0.50)\end{array}$ & $\begin{array}{l}\text { low } \\
(\text { WER }=1.55-1.75)\end{array}$ \\
\hline $10 \mathrm{~mm}$ & $\begin{array}{l}\text { thickly pachyconic; subevolute } \\
(\mathrm{ww} / \mathrm{dm}=0.75-0.80 ; \mathrm{uw} / \mathrm{dm}=0.30-0.38)\end{array}$ & $\begin{array}{l}\text { strongly depressed; strongly embracing } \\
(w w / w h=2.00-2.25 ; I Z R=0.35-0.40)\end{array}$ & $\begin{array}{l}\text { low } \\
(\text { WER }=1.55-1.75)\end{array}$ \\
\hline $30 \mathrm{~mm}$ & $\begin{array}{l}\text { thickly discoidal to thinly pachyconic; involute to sub- } \\
\text { involute }(\mathrm{ww} / \mathrm{dm}=0.55-0.65 ; \mathrm{uw} / \mathrm{dm}=0.10-0.20)\end{array}$ & $\begin{array}{l}\text { weakly depressed; strongly embracing } \\
(\mathrm{ww} / \mathrm{wh}=1.00-1.50 ; \mathrm{IZR}=0.40-0.45)\end{array}$ & $\begin{array}{l}\text { moderate to high } \\
(\text { WER }=1.90-2.10)\end{array}$ \\
\hline $60 \mathrm{~mm}$ & $\begin{array}{l}\text { thinly to thickly discoidal; involute } \\
(\mathrm{ww} / \mathrm{dm}=0.45-0.50 ; \mathrm{uw} / \mathrm{dm}=0.05-0.10)\end{array}$ & $\begin{array}{l}\text { weakly compressed; strongly embracing } \\
\text { (ww/wh }=0.80-1.00 ; \mathrm{IZR}=0.40-0.45 \text { ) }\end{array}$ & $\begin{array}{l}\text { high } \\
(\text { WER }=2.00-2.15)\end{array}$ \\
\hline
\end{tabular}

Table 28. Conch dimensions (in $\mathrm{mm}$ ) and proportions for reference specimens of Trimorphoceras crassilens $\mathrm{n}$. $\mathrm{sp}$.

\begin{tabular}{lcccccccccc}
\hline & $\mathrm{dm}$ & ww & wh & uw & ah & ww/dm & ww/wh & uw/dm & WER & IZR \\
\hline paratype MB.C.18610.11 & 67.8 & 32.2 & 36.6 & 5.4 & 21.4 & 0.47 & 0.88 & 0.08 & 2.13 & 0.42 \\
paratype MB.C.18610.10 & 59.0 & 27.7 & 32.9 & 3.6 & 18.3 & 0.47 & 0.84 & 0.06 & 2.10 & 0.44 \\
paratype MB.C.18610.2 & 51.5 & 26.1 & 27.8 & 3.3 & 15.1 & 0.51 & 0.94 & 0.06 & 2.00 & 0.46 \\
paratype MB.C.18610.4 & 46.4 & 26.6 & 26.4 & 2.6 & 13.0 & 0.57 & 1.01 & 0.05 & 1.93 & 0.51 \\
holotype MB.C.18610.1 & 45.8 & 23.5 & 25.8 & 3.5 & 13.6 & 0.51 & 0.91 & 0.08 & 2.02 & 0.47 \\
paratype MB.C.18610.12 & 38.7 & 21.5 & 20.8 & 3.7 & 12.1 & 0.56 & 1.03 & 0.09 & 2.12 & 0.42 \\
paratype MB.C.18610.3 & 32.1 & 18.8 & 16.6 & 4.2 & 8.3 & 0.58 & 1.13 & 0.13 & 1.81 & 0.50 \\
paratype MB.C.18610.13 & 20.1 & 14.2 & 9.3 & 4.8 & 6.1 & 0.71 & 1.52 & 0.24 & 2.05 & 0.35 \\
\hline
\end{tabular}

Table 29. Suture line proportions (Figs $30 \mathrm{H}-\mathrm{K}$ ) for Trimorphoceras crassilens $\mathrm{n}$. $\mathrm{sp}$.

\begin{tabular}{llllllll}
\hline specimen & at dm & ELw/d & EL/VLS & EL/AL & MS h & VLS w/h & remarks \\
\hline paratype MB.C.18610.10 & $54.5 \mathrm{~mm}$ & 0.42 & 0.50 & 1.00 & 0.21 & 0.85 & A lobe with almost straight flanks \\
holotype MB.C.18610.1 & $44.8 \mathrm{~mm}$ & 0.39 & 0.43 & 0.79 & 0.18 & 0.89 & A lobe with slightly curved flanks \\
paratype MB.C.18610.4 & $32.7 \mathrm{~mm}$ & 0.41 & 0.52 & 0.91 & 0.24 & 0.79 & A lobe asymmetric \\
paratype MB.C.18610.3 & $25.0 \mathrm{~mm}$ & 0.42 & 0.58 & 0.87 & 0.23 & 0.72 & A lobe asymmetric \\
\hline
\end{tabular}

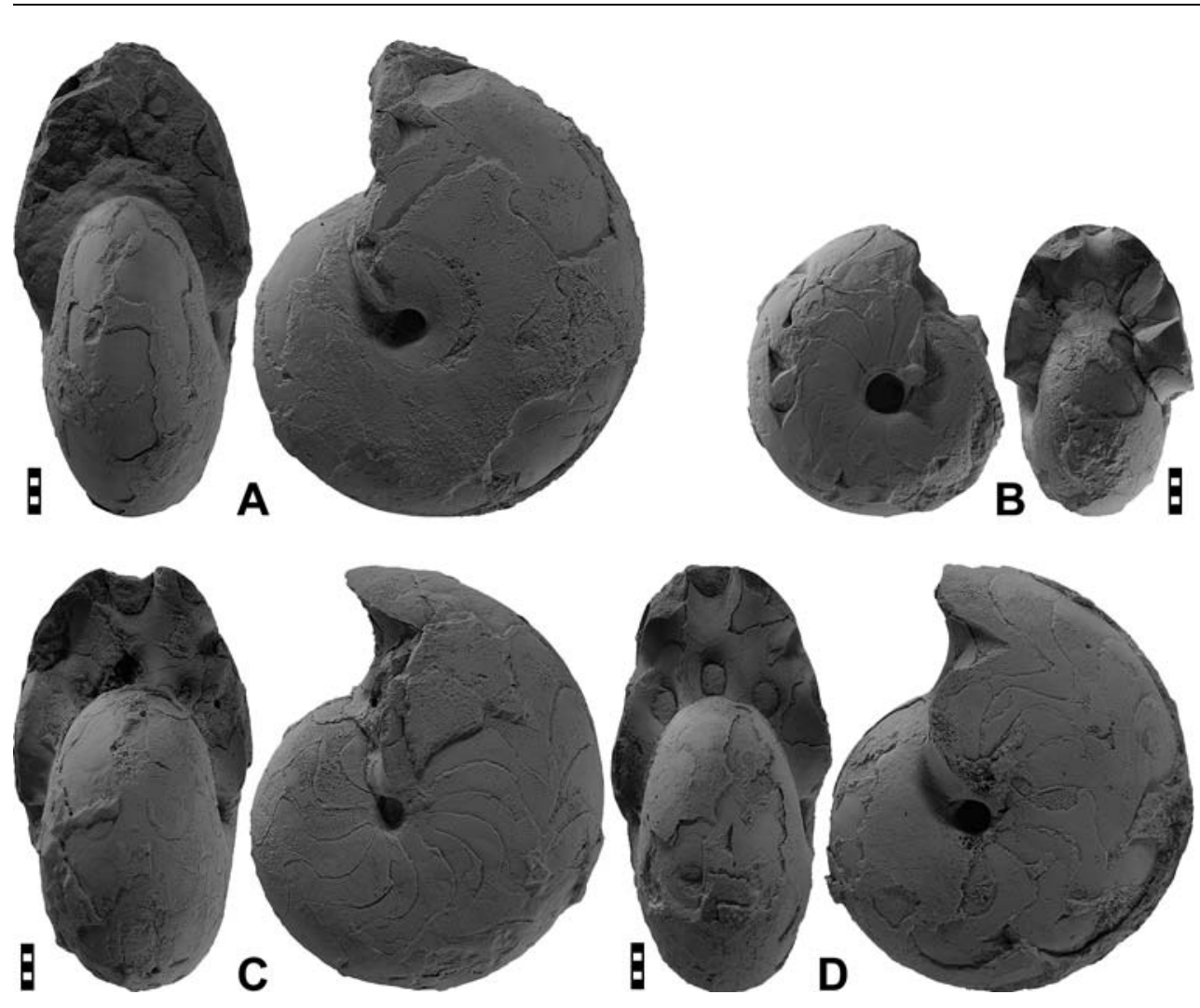

Figure 29. Trimorphoceras crassilens $\mathrm{n}$. sp. from locality TIM-C7; all $\times 1.0$. A. Paratype MB.C.18610.2. B. Paratype MB.C.18610.3. C. Paratype MB.C.18610.4 D. Holotype MB.C.18610.1. 
Discussion. The very strange ontogenetic development of the conch morphology makes Trimorphoceras crassilens a species easily separable from most of the other species from the Dalle à Merocanites when cross sections are available. Only T. absolutum is similar, but this species has wider whorls at comparable stages $(\mathrm{ww} / \mathrm{wh}=1.80-$ 2.20 at $15 \mathrm{~mm} \mathrm{dm}$ in T. absolutum and only 1.70-2.00 in T. crassilens). Whorl overlap upon the umbilicus is much less conspicuous in T. absolutum, and the external lobe is slightly pouched in this species.
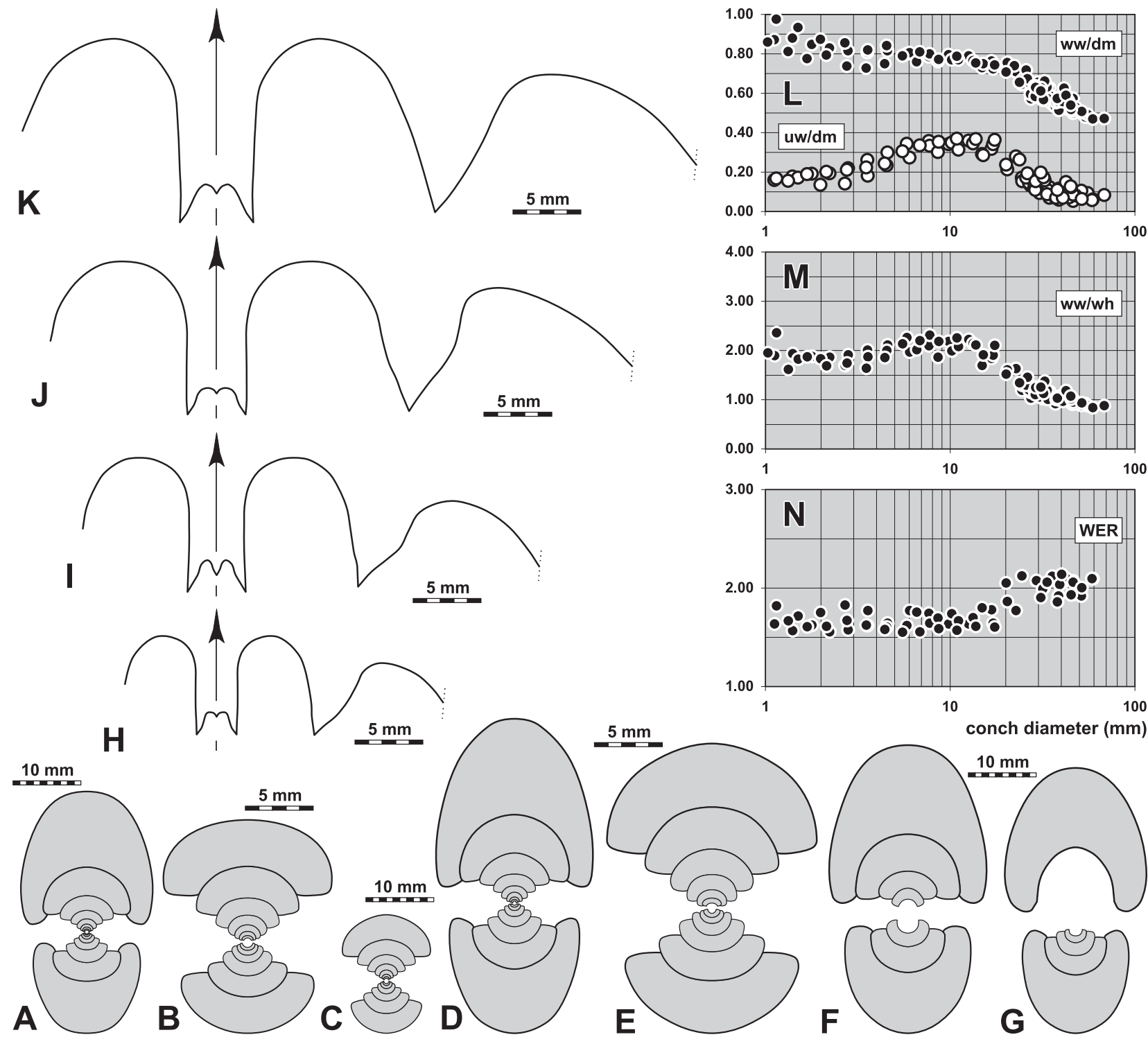

B
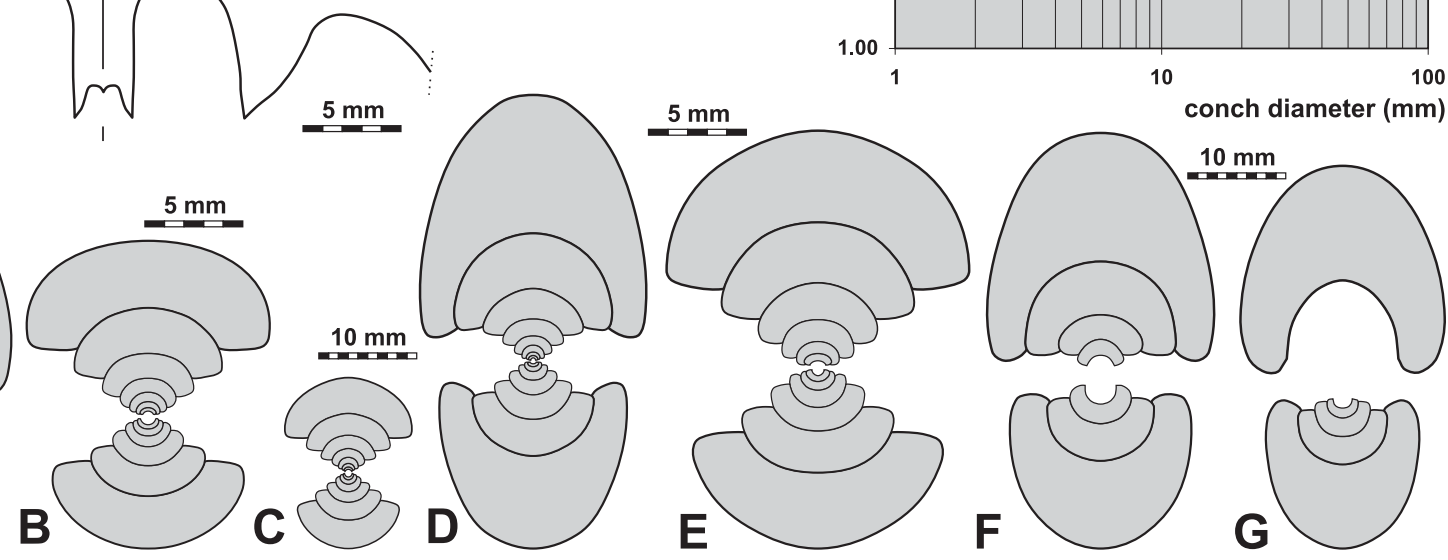

Figure 30. Trimorphoceras crassilens $\mathrm{n}$. sp. from locality TIM-C7. A. Cross section of paratype MB.C.18610.5; $\times 1.0$. B. Cross section (inner whorls) of the same specimen; $\times 2.5$. C. Cross section of paratype MB.C.18610.6; $\times 1.0$. D. Cross section of paratype MB.C. $18610.7 ; \times 1.0$. E. Cross section (inner whorls) of the same specimen; $\times 2.5$. F. Cross section of paratype MB.C.18610.8; $\times 1.0$. G. Cross section of paratype MB.C.18610.9; $\times 1.0$. H. Suture line of paratype MB.C.18610.3, at $25.0 \mathrm{~mm}$ dm, $15.2 \mathrm{~mm}$ ww, $12.1 \mathrm{~mm}$ wh; $\times 2.0$. I. Suture line of paratype MB.C. 18610.4 , at $32.7 \mathrm{~mm}$ ww, $20.8 \mathrm{~mm}$ ww, $18.5 \mathrm{~mm} \mathrm{wh;} \times 2.0$. J. Suture line of holotype MB.C.18610.1, at $44.8 \mathrm{~mm} \mathrm{dm}, 22.9 \mathrm{~mm}$ ww, $24.5 \mathrm{~mm}$ wh; $\times 2.0$. K. Suture line of paratype MB.C.18610.10, at $54.5 \mathrm{~mm} \mathrm{dm}$, $27.4 \mathrm{~mm}$ ww, $29.2 \mathrm{~mm}$ wh; $\times 2.0$. L-N. Ontogenetic development of the conch width index (ww/dm), umbilical width index (uw/ $\mathrm{dm}$ ), whorl width index (ww/wh), and whorl expansion rate (WER) of all available specimens. 
Muensteroceras fabrei is very similar in the growth stage between 40 and $60 \mathrm{~mm}$ conch diameter, but possesses a slightly wider umbilicus. At $30-40 \mathrm{~mm} \mathrm{dm}$, the umbilicus is opening in M. fabrei, but in T. crassilens it is in the process of closure. The best criterion to separate between the two species is, if the conch ontogeny is not known, the external lobe, which is slightly pouched in T. crassilens (M. fabrei has parallel or slightly diverging flanks of the external lobe).

Another co-occurring species with a very similar conch in the adult stage is Dzhaprakoceras dzhazairense, and confusion of the two species is possible when the development of the umbilicus is not known. T. crassilens shows the striking closure, by significant overlap, of the umbilicus, in contrast to the continuously opening umbilicus in D. dzhazairense.

\section{Trimorphoceras absolutum n. sp.}

Figures 31, 32

Derivation of name. After Lat. absolutum $=$ complete, because of the completely preserved ontogeny in the material.

Holotype. Specimen MB.C.18611.1, illustrated in Figure 31B.

Type locality and horizon. Sebkha de Timimoun, locality TIM-C7 (14.5 km west-southwest of Timimoun, Algeria); Ammonellipsites-Merocanites Assemblage.

Material. Well preserved material is available; in total 47 specimens between 30 and $66 \mathrm{~mm}$ conch diameter were studied. The maximum diameter with body chamber can be estimated to be about $95 \mathrm{~mm}$.

Diagnosis. Trimorphoceras with a thickly pachyconic conch between 2 and $22 \mathrm{~mm} \mathrm{dm}$, thereafter rapid lateral compression towards a thickly discoidal conch at $60 \mathrm{~mm} \mathrm{dm}$; juvenile stage subinvolute, growth interval between 15 and $20 \mathrm{~mm} \mathrm{dm}$ subevolute, rapid closure of the umbilicus in the intermediate stage by strong whorl overlap; umbilical margin rounded throughout ontogeny; umbilical wall convex; aperture low or moderately high in juveniles and rapidly becoming high in growth stages above $20 \mathrm{~mm} \mathrm{dm}$. Ornament with very fine growth lines; course concavo-convex with very shallow lateral sinus and deep ventral sinus, direction rectiradiate; small specimens with shell constrictions parallel to the growth lines, specimens larger than $30 \mathrm{~mm}$ dm without constrictions on shell and steinkern. Suture line with narrow, parallel-sided external lobe and low median saddle; ventrolateral saddle broadly rounded and almost symmetric; adventive lobe V-shaped, slightly asymmetric.
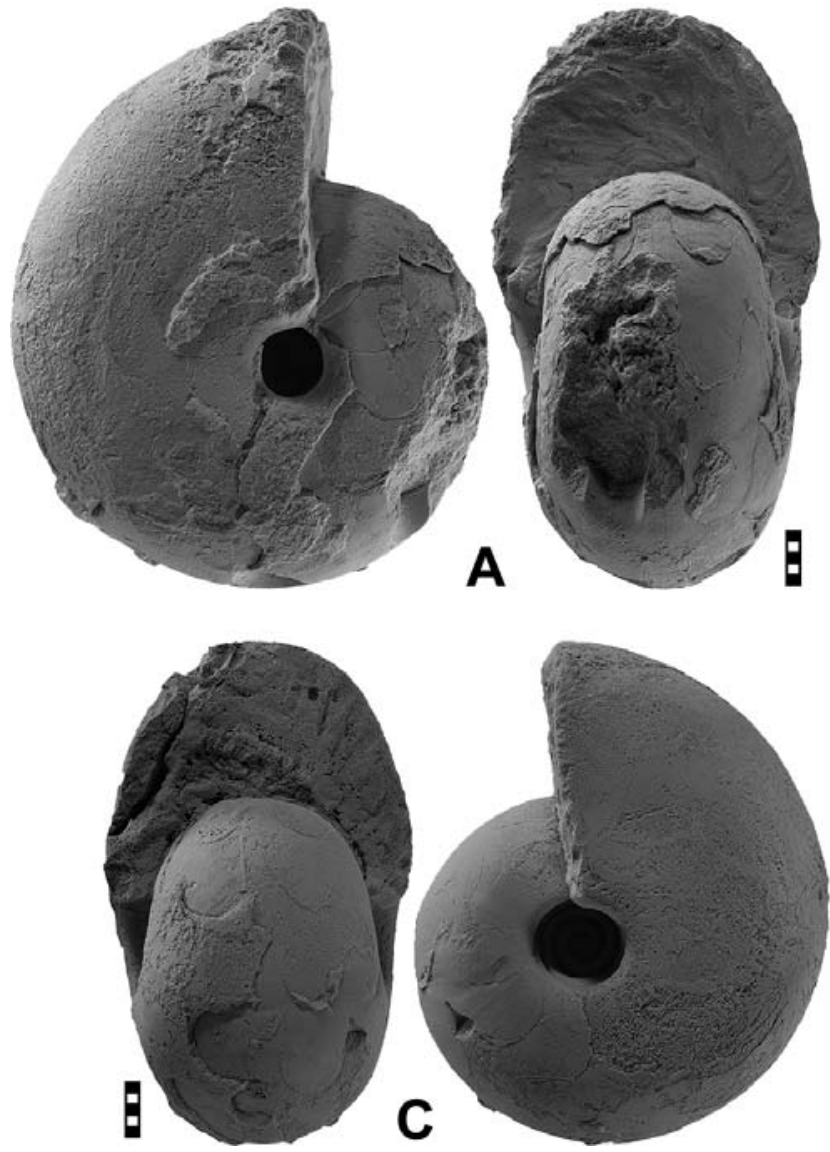
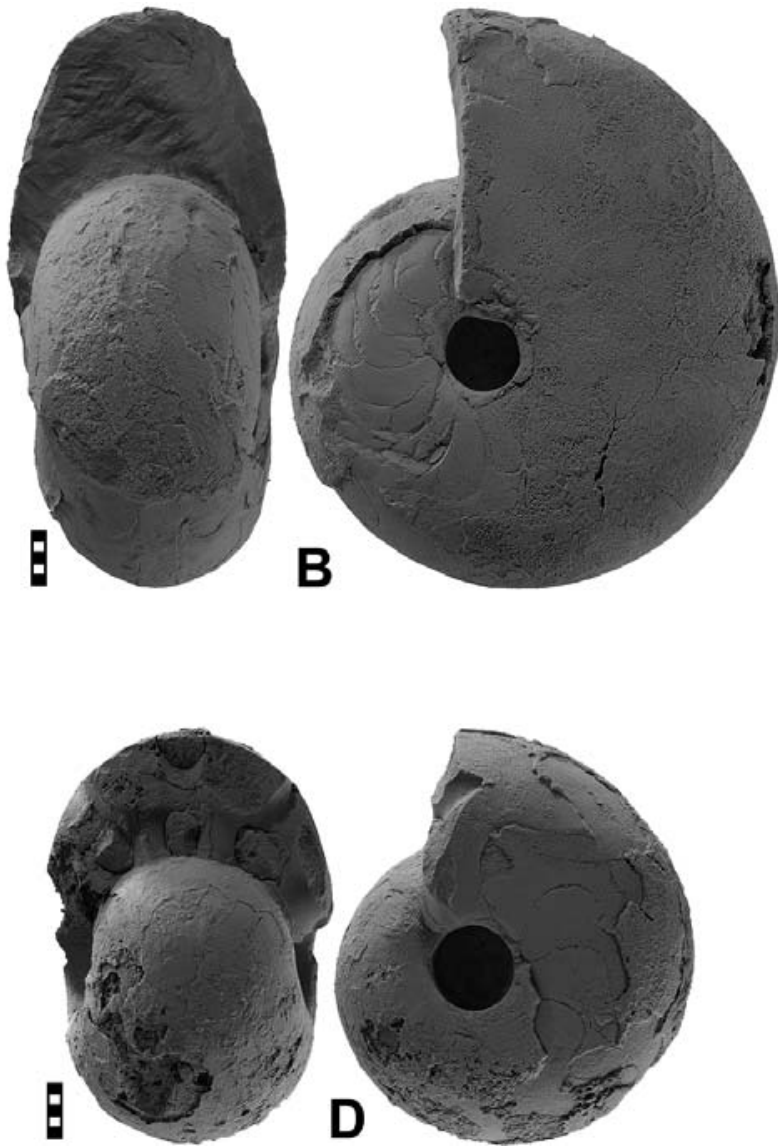

Figure 31. Trimorphoceras absolutum n. sp. from locality TIM-C7; all $\times 1.0$. A. Paratype MB.C.18611.2. B. Holotype MB.C.18611.1. C. Paratype MB.C.18611.3. D. Paratype MB.C.18611.12. 
Table 30. Conch ontogeny (Figs 32A-E, I-K) of Trimorphoceras absolutum n. sp.

\begin{tabular}{|c|c|c|c|}
\hline$d m$ & conch shape & whorl cross section shape & aperture \\
\hline $4 \mathrm{~mm}$ & $\begin{array}{l}\text { thinly to thickly pachyconic; involute to } \\
\text { subinvolute ( } w w / d m=0.70-0.80 ; u w / d m=0.10-0.20 \text { ) }\end{array}$ & $\begin{array}{l}\text { weakly to moderately depressed; very strongly embra- } \\
\text { cing (ww/wh }=1.40-1.70 ; \text { IZR }=0.45-0.50 \text { ) }\end{array}$ & $\begin{array}{l}\text { moderate } \\
(\mathrm{WER}=1.75-2.00)\end{array}$ \\
\hline $10 \mathrm{~mm}$ & $\begin{array}{l}\text { thinly to thickly pachyconic; subinvolute to subevolute } \\
\text { (ww/dm }=0.70-0.80 ; u w / d m=0.25-0.35 \text { ) }\end{array}$ & $\begin{array}{l}\text { moderately to strongly depressed; strongly embracing } \\
\text { (ww/wh }=1.70-2.10 ; \text { IZR }=0.40-0.45 \text { ) }\end{array}$ & $\begin{array}{l}\text { low to moderate } \\
\text { (WER }=1.60-1.80)\end{array}$ \\
\hline $30 \mathrm{~mm}$ & $\begin{array}{l}\text { thinly pachyconic; subinvolute } \\
\text { (ww/dm }=0.60-0.70 ; \text { uw/dm }=0.20-0.30 \text { ) }\end{array}$ & $\begin{array}{l}\text { weakly to moderately depressed; very strongly embra- } \\
\text { cing (ww/wh }=1.40-1.70 ; \mathrm{IZR}=0.45-0.50 \text { ) }\end{array}$ & $\begin{array}{l}\text { moderate } \\
(\mathrm{WER}=1.75-2.00)\end{array}$ \\
\hline $60 \mathrm{~mm}$ & $\begin{array}{l}\text { thickly discoidal; involute } \\
(\mathrm{ww} / \mathrm{dm}=0.50-0.60 ; \mathrm{w} / \mathrm{dm}=0.10-0.15)\end{array}$ & $\begin{array}{l}\text { weakly compressed to weakly depressed; strongly } \\
\text { embracing (ww/wh }=0.80-1.15 ; \mathrm{IZR}=0.40-0.45 \text { ) }\end{array}$ & $\begin{array}{l}\text { moderate to high } \\
(\mathrm{WER}=1.90-2.15)\end{array}$ \\
\hline
\end{tabular}

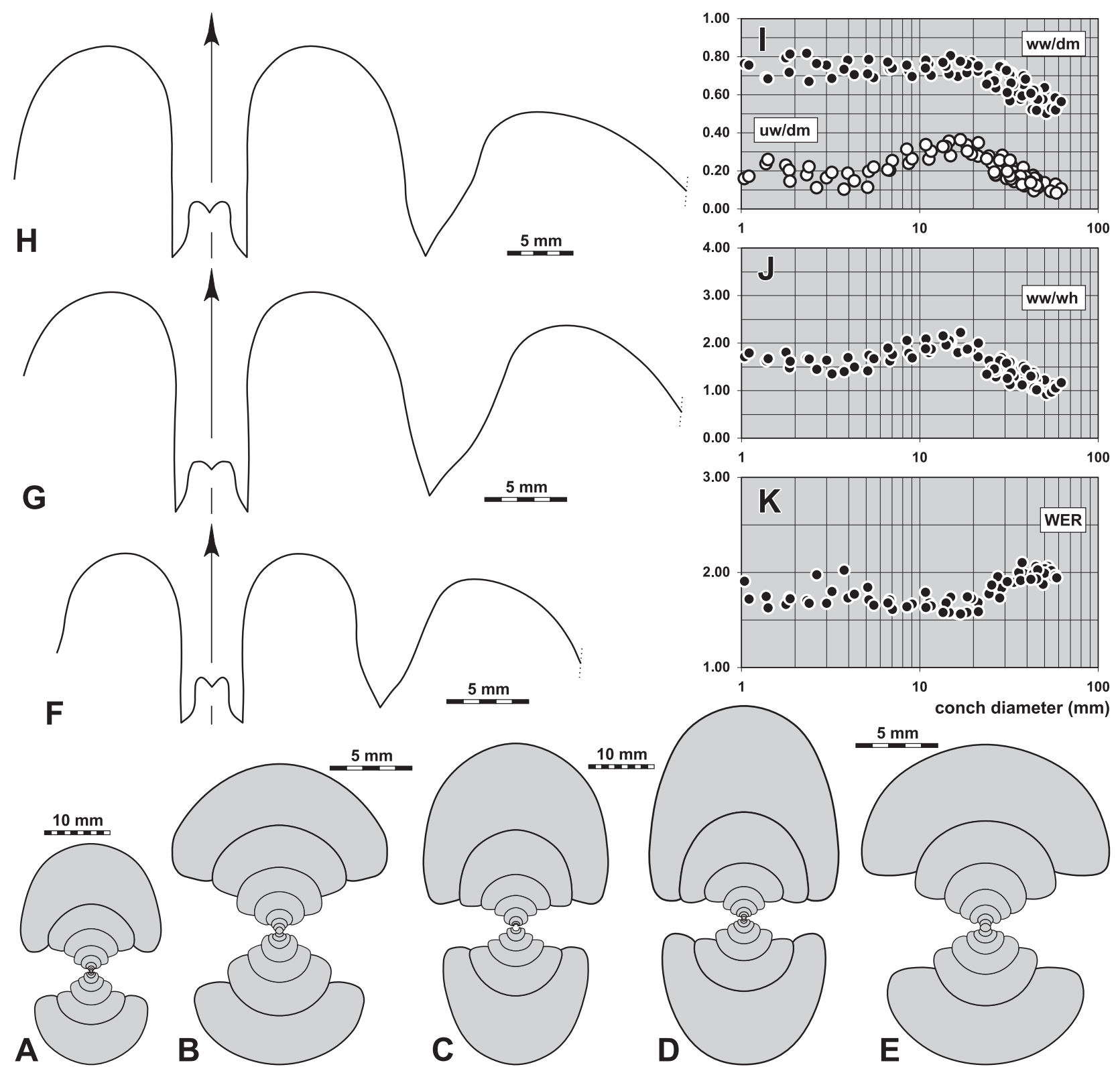

Figure 32. Trimorphoceras absolutum n. sp. from locality TIM-C7. A. Cross section of paratype MB.C.18611.4; $\times 1.0$. B. Cross section (inner whorls) of the same specimen; $\times 2.5$. C. Cross section of paratype MB.C.18611.5; $\times 1.0$. D. Cross section of paratype MB.C.18611.6; $\times 1.0$. E. Cross section (inner whorls) of the same specimen; $\times 2.5$. F. Suture line of holotype MB.C.18611.1, at $37.5 \mathrm{~mm} \mathrm{dm}, 21.7 \mathrm{~mm}$ ww, $17.2 \mathrm{~mm}$ wh; $\times 2.5$. G. Suture line of paratype MB.C.18611.7, at $42.4 \mathrm{~mm} \mathrm{dm}, 24.6 \mathrm{~mm}$ ww, $21.8 \mathrm{~mm}$ wh; $\times 2.5$. H. Suture line of paratype MB.C.18611.8, at $57.9 \mathrm{~mm} \mathrm{dm}, 31.0 \mathrm{~mm} w w, 29.0 \mathrm{~mm}$ wh; $\times 2.0$. I-K. Ontogenetic development of the conch width index (ww/dm), umbilical width index (uw/dm), whorl width index (ww/wh), and whorl expansion rate (WER) of all available specimens. 
Table 31. Conch dimensions (in $\mathrm{mm}$ ) and proportions for reference specimens of Trimorphoceras absolutum n. sp.

\begin{tabular}{|c|c|c|c|c|c|c|c|c|c|c|}
\hline & $\mathrm{dm}$ & ww & wh & uw & ah & $w w / d m$ & ww/wh & uw/dm & WER & IZR \\
\hline paratype MB.C.18611.9 & 65.8 & 35.9 & 31.8 & 6.1 & 18.5 & 0.55 & 1.13 & 0.09 & 1.93 & 0.42 \\
\hline paratype MB.C.18611.8 & 58.7 & 31.3 & 28.7 & 7.0 & 16.6 & 0.53 & 1.09 & 0.12 & 1.94 & 0.42 \\
\hline paratype MB.C.18611.2 & 52.2 & 29.0 & 27.0 & 6.0 & 15.9 & 0.56 & 1.08 & 0.12 & 2.06 & 0.41 \\
\hline holotype MB.C.18611.1 & 51.2 & 25.7 & 28.0 & 6.4 & 14.6 & 0.50 & 0.92 & 0.13 & 1.96 & 0.48 \\
\hline paratype MB.C.18611.10 & 50.2 & 30.7 & 25.6 & 6.8 & 15.1 & 0.61 & 1.20 & 0.13 & 2.04 & 0.41 \\
\hline paratype MB.C.18611.3 & 45.4 & 27.8 & 23.2 & 7.0 & 12.9 & 0.61 & 1.20 & 0.15 & 1.95 & 0.45 \\
\hline paratype MB.C.18611.11 & 43.1 & 26.7 & 19.4 & 7.6 & 12.5 & 0.62 & 1.38 & 0.18 & 1.98 & 0.35 \\
\hline paratype MB.C.18611.12 & 37.0 & 24.4 & 17.9 & 7.5 & 10.3 & 0.66 & 1.36 & 0.20 & 1.92 & 0.43 \\
\hline
\end{tabular}

Table 32. Suture line proportions (Figs $32 \mathrm{~F}-\mathrm{H}$ ) for specimens of Trimorphoceras absolutum $\mathrm{n} . \mathrm{sp}$

\begin{tabular}{llllllll}
\hline specimen & at dm & EL w/d & EL/NLS & EL/AL & MS h & VLS w/h & remarks \\
\hline paratype MB.C.18611.8 & $57.9 \mathrm{~mm}$ & 0.35 & 0.49 & 0.93 & 0.26 & 0.73 & A lobe rather narrow \\
paratype MB.C.18611.7 & $42.4 \mathrm{~mm}$ & 0.32 & 0.45 & 0.78 & 0.23 & 0.71 & A lobe V-shaped, symmetric \\
paratype MB.C.18611.11 & $40.0 \mathrm{~mm}$ & 0.35 & 0.45 & 0.94 & 0.25 & 0.79 & 0.67 \\
holotype MB.C.18611.1 & $37.5 \mathrm{~mm}$ & 0.37 & 0.54 & 0.94 & 0.27 & 0.67 \\
paratype MB.C.18611.3 & c. $35.0 \mathrm{~mm}$ & 0.34 & 0.51 & 0.96 & 0.27 & 0.67 \\
\hline
\end{tabular}

Discussion. Trimorphoceras absolutum has a morphological position between T. crassilens and T. molestum. It differs in the slightly stouter conch and the less conspicuous whorl overlap onto the umbilicus in the adult stage from T. crassilens. Therefore, the uw/dm ratio amounts to 0.05 in T. crassilens at $45 \mathrm{~mm}$ conch diameter but to 0.15 in T. absolutum. T. molestum and T. sphaeroidalis (M'Coy, 1844) have a stouter conch; T. sphaeroidalis has a wider umbilicus (uw/dm $>0.20$ at $45 \mathrm{~mm} \mathrm{dm}$ ).

\section{Trimorphoceras molestum n. sp.}

Figures 33, 34

Derivation of name. After Lat. molestum = difficult, because of the difficult separation from other species.

Holotype. Specimen MB.C.18612.1, illustrated in Figure 33A.

Type locality and horizon. Sebkha de Timimoun, locality TIM-C7 (14.5 km west-southwest of Timimoun, Algeria); Ammonellipsites-Merocanites Assemblage.

Material. Well-preserved material is available; 41 specimens between 27 and $80 \mathrm{~mm}$ conch diameter were studied. The maximum diameter with body chamber can be estimated with $100 \mathrm{~mm}$.

Diagnosis. Trimorphoceras with a thickly pachyconic conch between 2 and $30 \mathrm{~mm} \mathrm{dm}$, thereafter lateral compression towards a thickly discoidal conch at $60 \mathrm{~mm} \mathrm{dm}$; juvenile stage at $15 \mathrm{~mm} \mathrm{dm}$ subevolute, closure of the umbilicus in the adult stage by moderate whorl overlap; umbilical margin rounded or subangular; umbilical wall convex; aperture low or moderately high in juveniles and high in growth stages above $20 \mathrm{~mm}$ dm. Ornament with very fine growth lines; course concavo-convex with very shallow lateral sinus and deep ventral sinus, direction rectiradiate; small specimens with shell constrictions parallel to the growth lines, specimens larger than $30 \mathrm{~mm}$ dm without constrictions on shell and steinkern. Suture line with narrow, parallel-sided external lobe and low median saddle; ventrolateral saddle broadly rounded and almost symmetric; adventive lobe V-shaped, almost symmetric.

Table 33. Conch ontogeny (Figs 34A-C, F-H) of Trimorphoceras molestum n. sp.

\begin{tabular}{llll}
\hline $\mathrm{dm}$ & conch shape & whorl cross section shape & aperture \\
\hline $10 \mathrm{~mm}$ & thinly pachyconic; subevolute & moderately depressed; strongly embracing & low \\
& $($ ww/dm $\sim 0.70 ;$ uw/dm $\sim 0.34)$ & $($ ww/wh $\sim 1.90 ;$ IZR $\sim 0.45)$ & moderately depressed; strongly embracing
\end{tabular}


Table 34. Conch dimensions (in $\mathrm{mm}$ ) and proportions for reference specimens of Trimorphoceras molestum $\mathrm{n}$. sp.

\begin{tabular}{lllllllllll}
\hline & $\mathrm{dm}$ & ww & wh & uw & ah & ww/dm & ww/wh & uw/dm & WER & IZR \\
\hline holotype MB.C.18612.1 & 64.2 & 36.3 & 31.9 & 12.3 & 18.5 & 0.56 & 1.14 & 0.19 & 1.97 & 0.42 \\
paratype MB.C.18612.4 & 61.6 & 34.1 & 31.0 & 10.3 & 17.9 & 0.55 & 1.10 & 0.17 & 1.99 & 0.42 \\
paratype MB.C.18612.10 & 57.2 & 39.6 & 29.0 & 9.2 & 17.7 & 0.69 & 1.36 & 0.16 & 2.10 & 0.39 \\
paratype MB.C.18612.5 & 54.6 & 36.1 & 27.5 & 8.8 & 16.4 & 0.66 & 1.31 & 0.16 & 2.04 & 0.41 \\
paratype MB.C.18612.11 & 54.5 & 34.7 & 25.5 & 8.6 & 15.3 & 0.64 & 1.36 & 0.16 & 1.94 & 0.40 \\
paratype MB.C.18612.2 & 53.8 & 36.1 & 26.7 & 8.8 & 15.4 & 0.67 & 1.35 & 0.16 & 1.97 & 0.42 \\
paratype MB.C.18612.12 & 50.2 & 32.1 & 26.7 & 8.4 & 14.2 & 0.64 & 1.30 & 0.17 & 1.95 & 0.42 \\
paratype MB.C.18612.13 & 43.3 & 30.5 & 21.6 & 7.3 & 12.3 & 0.70 & 1.41 & 0.17 & 1.95 & 0.43 \\
paratype MB.C.18612.3 & 32.8 & 23.5 & 14.8 & 7.8 & 8.5 & 0.72 & 1.59 & 0.24 & 1.82 & 0.43 \\
\hline
\end{tabular}

Table 35. Suture line proportions (Figs 34D, E) for specimens of Trimorphoceras molestum $\mathrm{n}$. sp.

\begin{tabular}{llllllll}
\hline specimen & at dm & EL w/d & EL/NLS & EL/AL & MS h & VLS w/h & remarks \\
\hline paratype MB.C.18612.9 & c. $40.0 \mathrm{~mm}$ & 0.32 & 0.47 & 0.77 & 0.22 & 0.67 & VL saddle ventrally inclined \\
paratype MB.C.18612.8 & c. $25.0 \mathrm{~mm}$ & 0.33 & 0.47 & 0.76 & 0.16 & 0.70 & VL saddle almost symmetric \\
\hline
\end{tabular}

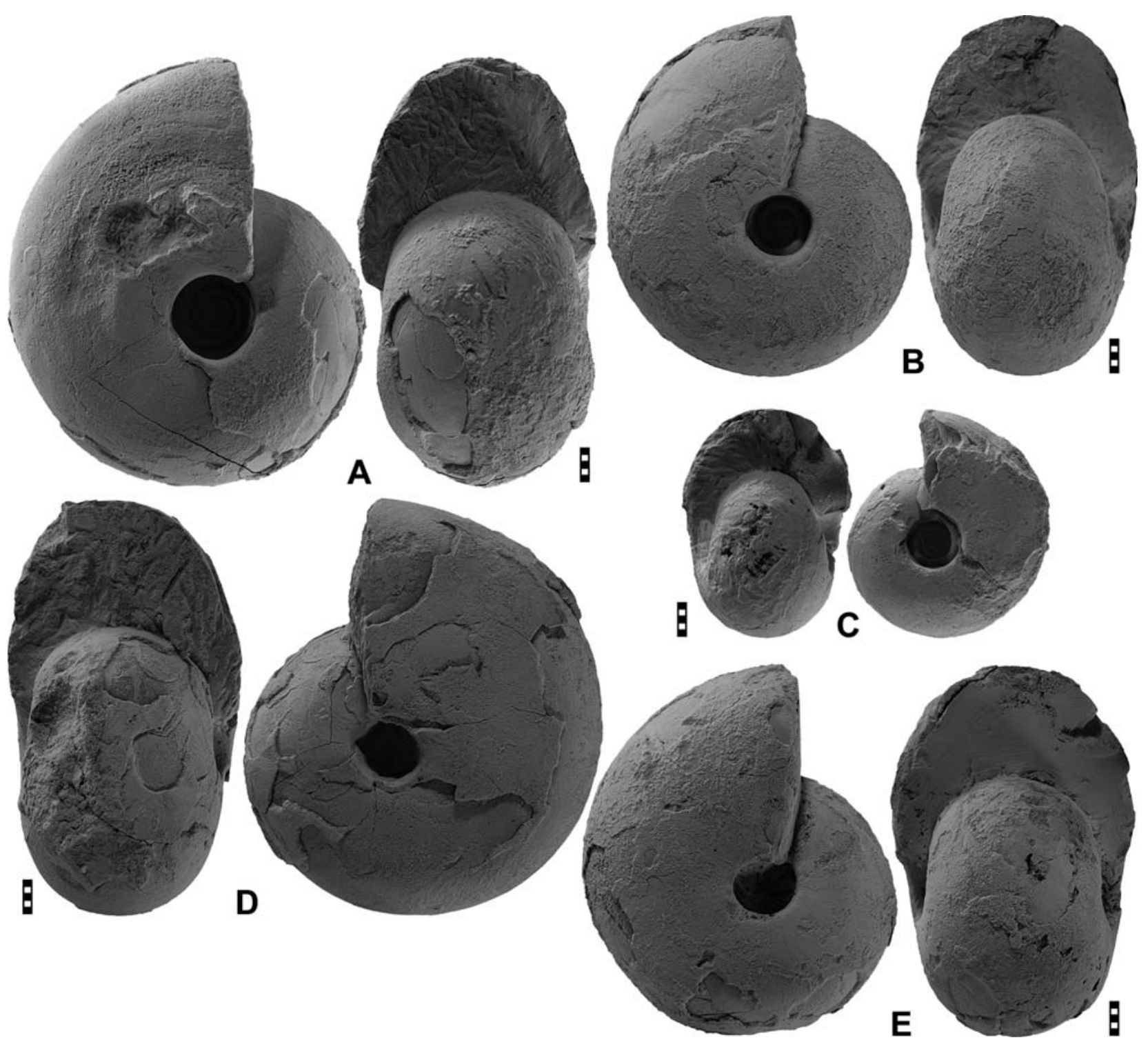

Figure 33. Trimorphoceras molestum n. sp. from locality TIM-C7; all $\times 1.0$. A. Holotype MB.C.18612.1. B. Paratype MB.C.18612.2. C. Paratype MB.C.18612.3. D. Paratype MB.C.18612.4. E. Paratype MB.C.18612.5. 
Discussion. Trimorphoceras absolutum is a similar species, but possesses a more slender conch and a narrower umbilicus than T. molestum; these differences are most obvious in the growth stage above $40 \mathrm{~mm}$ conch diameter. Furthermore, the external lobe is narrower in T. molestum, and the ventrolateral saddle is more asymmetric. T. sphaeroidale (M'Coy, 1844) has a less stout conch shape and a wider umbilicus at comparable growth stages.
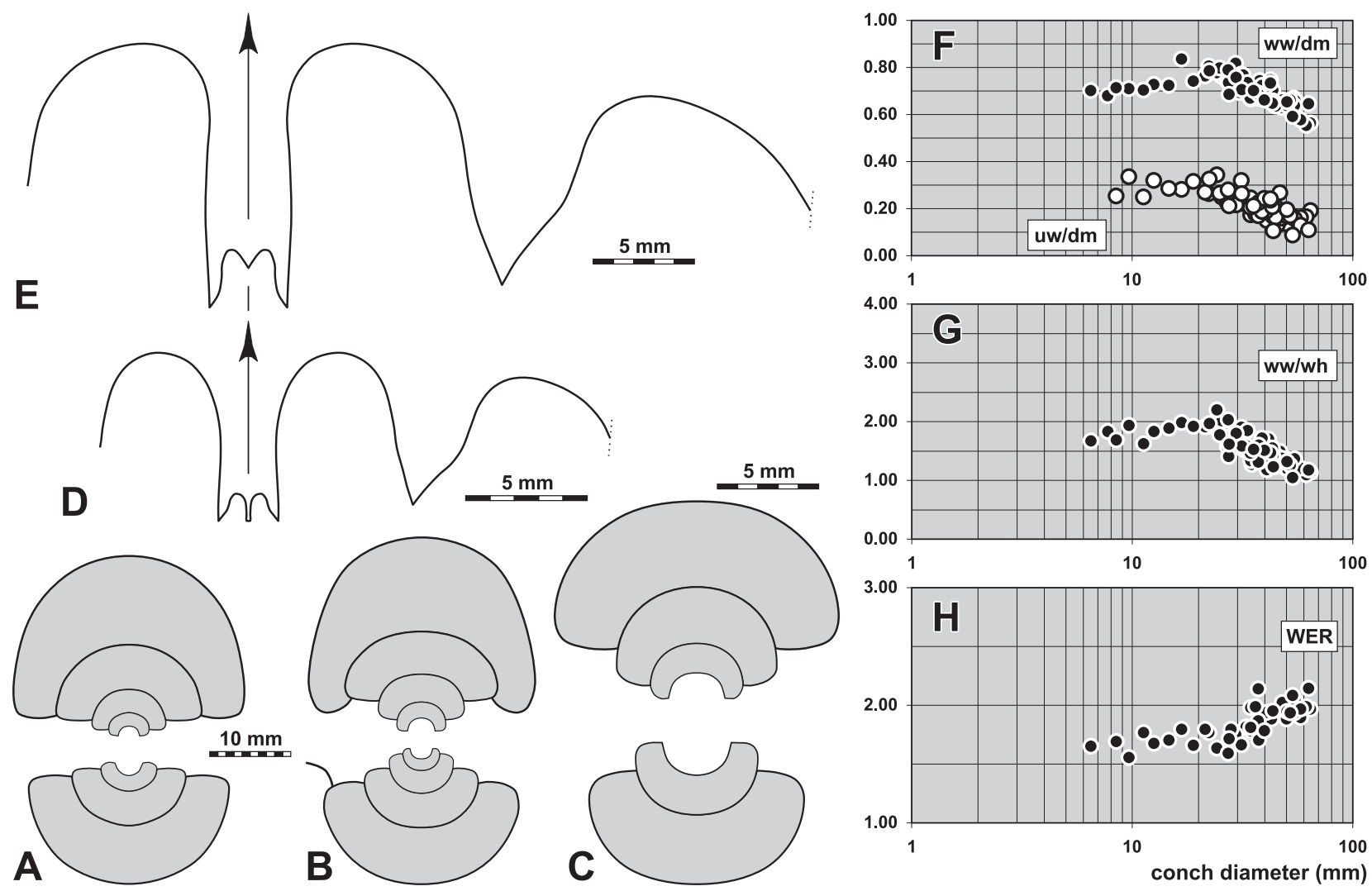

Figure 34. Trimorphoceras molestum n. sp. from locality TIM-C7. A. Cross section of paratype MB.C.18612.6; $\times 1.0$. B. Cross section of paratype MB.C.18612.7; $\times 1.0$. C. Cross section (inner whorls) of the same specimen; $\times 2.5$. D. Suture line of paratype MB.C.18612.8, at $18.4 \mathrm{~mm}$ ww, $9.4 \mathrm{~mm}$ wh; $\times 3.0$. E. Suture line of paratype MB.C.18612.9, at $29.6 \mathrm{~mm} w w, 20.2 \mathrm{~mm}$ wh; $\times 2.5$. F-H. Ontogenetic development of the conch width index (ww/dm), umbilical width index (uw/dm), whorl width index (ww/wh), and whorl expansion rate (WER) of all available specimens.

\section{Obturgites n. gen.}

Derivation of name. After Lat. obturgesco = swelling, because of the rapid ontogenetic increase of the whorl width.

Type species. Obturgites polysarcus n. sp.

Genus definition. Muensteroceratinae with insignificant ontogenetic changes of the conch geometry; shape pachyconic or globular and involute or subinvolute with ontogenetic development towards a stouter adult conch by rapid increasing rates of the whorl width (Fig. 35); flanks converge strongly towards the rounded venter; aperture moderately high to high; umbilical wall often flattened, umbilical margin narrowly rounded or subangular. Ornament with extremely fine growth lines; without constrictions. Suture line with very narrow, parallel-sided or slightly pouched external lobe and low median saddle; ventrolateral saddle broadly rounded; adventive lobe V-shaped, asymmetric or symmetric.

Included species.

polysarcus: Obturgites polysarcus n. sp.; Gourara, Algeria.

oligosarcus: Obturgites oligosarcus n. sp.; Gourara, Algeria.

Separation of the new species. The two species of Obturgites from the Dalle à Merocanites can easily be separated by their conch shape at 30$50 \mathrm{~mm} \mathrm{dm:}$

- Obturgites polysarcus - conch thickly discoidal $(\mathrm{ww} / \mathrm{dm}=0.75-0.80)$;

- Obturgites oligosarcus - conch thinly discoidal $(\mathrm{ww} / \mathrm{dm}=0.60-0.75)$.

Discussion. Obturgites differs from Eurites mainly in the ontogenetic development of the conch, which in Eurites includes a temporary accelerated opening of the umbilicus in the middle growth stage, whereas the umbilicus in Obturgites shows an almost continuous and slow opening rate. Furthermore, Obturgites differs in the much stronger increasing rate of the whorl width in some ontogenetic stages. Species may be difficult to assign to either of the two genera when their ontogeny is not known. 

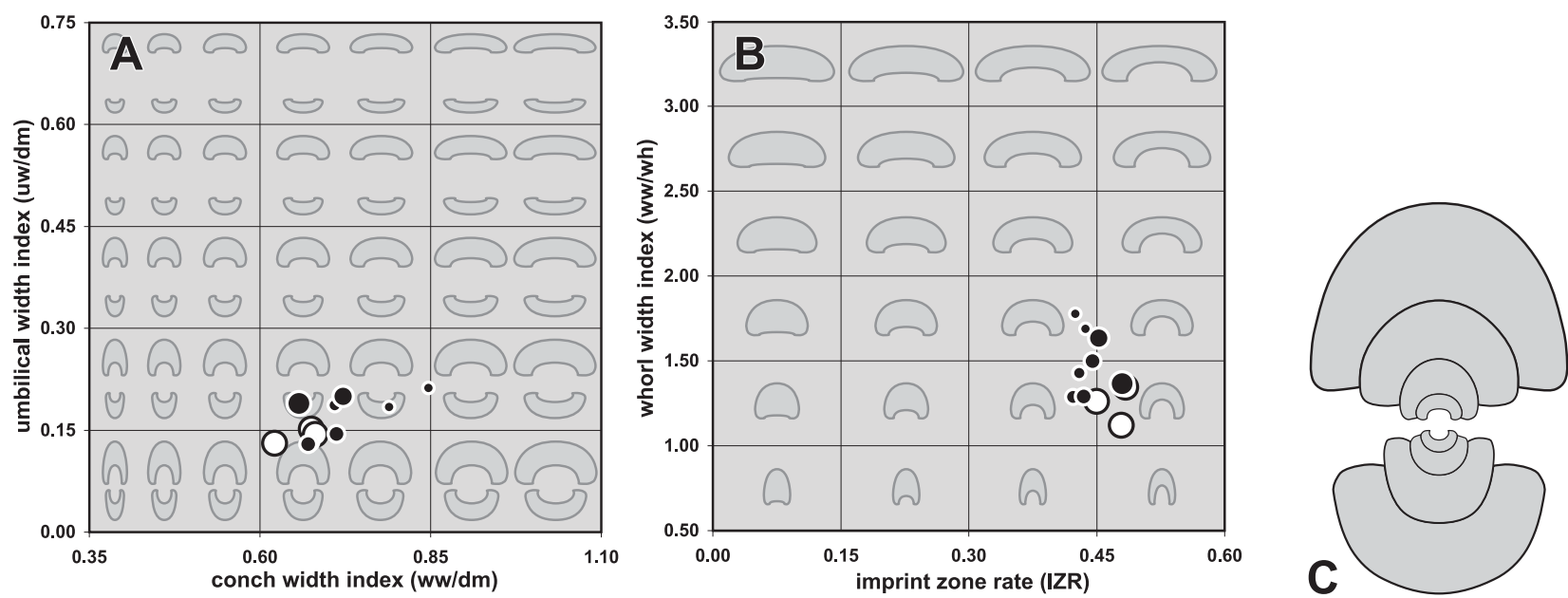

Figure 35. Ontogenetic trajectories of Obturgites, exemplified for Obturgites oligosarcus n. sp. from locality TIM-C7. A. Ontogenetic development of the conch width index (ww/dm), umbilical width index (uw/dm). B. Ontogenetic development of the imprint zone rate (IZR), whorl width index (ww/wh). C. Cross section of paratype MB.C.18614.4; $\times 1.0$. [Black dots represent ontogenetic stages of cross section MB.C.18614.4, white dots represent the reference specimens (Tab. 40).]

\section{Obturgites polysarcus $\mathbf{n}$. sp.}

Figures 36, 37

Derivation of name. After Greek poly = much and sarcus $=$ flesh, because of the voluminous body chamber. Holotype. Specimen MB.C.18613.1, illustrated in Figure 36C.

Type locality and horizon. Sebkha de Timimoun, locality TIM-C7 (14.5 km west-southwest of Timimoun, Algeria); Ammonellipsites-Merocanites Assemblage.
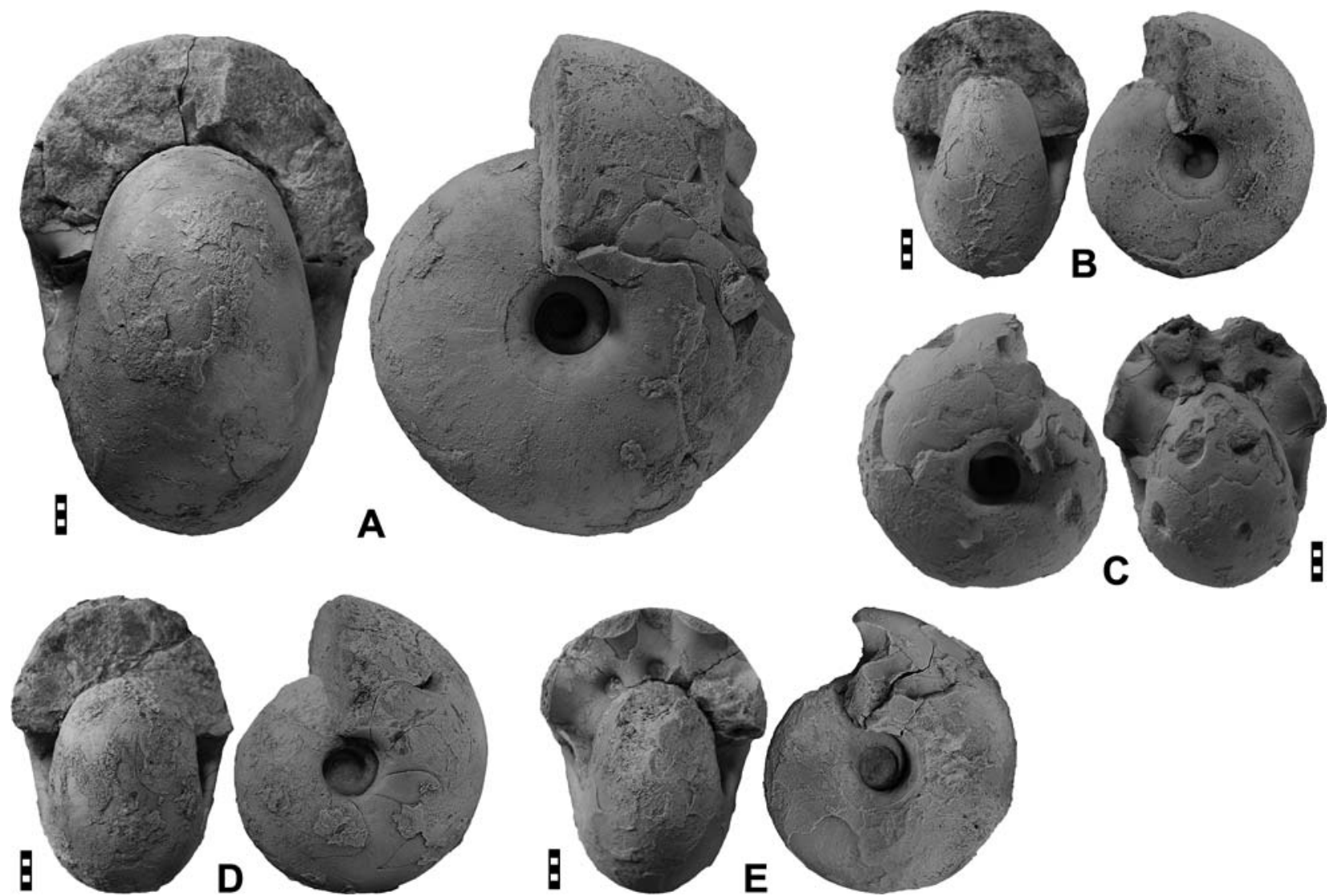

Figure 36. Obturgites polysarcus n. sp. from locality TIM-C7; all ×1.0. A. Paratype MB.C.18613.2. B. Paratype MB.C.18613.3. C. Holotype MB.C.18613.1. D. Paratype MB.C.18613.4. E. Paratype MB.C.18613.5. 
Material. A total of 25 specimens between 20 and $93 \mathrm{~mm}$ diameter are available. The largest specimen is paratype MB.C.18613.9; it is fully chambered at $93 \mathrm{~mm} \mathrm{dm}$, suggesting a total conch diameter with body chamber of approximately $170 \mathrm{~mm}$.

Diagnosis. Obturgites with a thickly pachyconic conch between 2 and $10 \mathrm{~mm} \mathrm{dm}$, thereafter lateral compression towards a thickly discoidal conch at $15-20 \mathrm{~mm} \mathrm{dm}$ and reversal to a thickly pachyconic adult conch; juvenile stage subinvolute, slight opening of the umbilicus in the adult stage; umbilical margin rounded or subangular; umbilical wall slightly flattened; aperture low or moderately high throughout ontogeny. Ornament with very fine growth lines; course concavo-convex with very shallow lateral sinus and deep ventral sinus, direction rectiradiate; small specimens with shell constrictions parallel to the growth lines, specimens larger than $30 \mathrm{~mm}$ dm without constrictions on shell and steinkern; very weak umbilical rim in the juvenile stages. Suture line with very narrow external lobe with slightly concave flanks and low median saddle; ventrolateral saddle broadly rounded; adventive lobe V-shaped, symmetric.

Table 36. Conch ontogeny (Figs 37A, B, E-G) of Obturgites polysarcus n. sp.

\begin{tabular}{|c|c|c|c|}
\hline$d m$ & conch shape & whorl cross section shape & aperture \\
\hline $10 \mathrm{~mm}$ & $\begin{array}{l}\text { thickly pachyconic; subinvolute } \\
\text { (ww/dm } \sim 0.74 ; \text { uw/dm } \sim 0.20 \text { ) }\end{array}$ & $\begin{array}{l}\text { weakly depressed; strongly embracing } \\
\text { (ww/wh } \sim 2.00 ; \text { IZR } \sim 0.45 \text { ) }\end{array}$ & $\begin{array}{l}\text { moderate } \\
(\mathrm{WER} \sim 1.90)\end{array}$ \\
\hline $30 \mathrm{~mm}$ & $\begin{array}{l}\text { thinly to thickly pachyconic; subinvolute } \\
\text { (ww/dm }=0.65-0.75 ; u w / d m=0.15-0.25 \text { ) }\end{array}$ & $\begin{array}{l}\text { moderately depressed; very strongly embracing } \\
(\mathrm{ww} / \mathrm{wh}=1.50-1.75 ; \mathrm{IZR}=0.45-0.50)\end{array}$ & $\begin{array}{l}\text { moderate } \\
(\mathrm{WER}=1.75-1.85)\end{array}$ \\
\hline $60 \mathrm{~mm}$ & $\begin{array}{l}\text { thickly pachyconic; subinvolute } \\
(\mathrm{ww} / \mathrm{dm}=0.70-0.80 ; \mathrm{uw} / \mathrm{dm}=0.15-0.20)\end{array}$ & $\begin{array}{l}\text { moderately depressed; very strongly embracing } \\
(\mathrm{ww} / \mathrm{wh}=1.50-1.60 ; \mathrm{IZR}=0.45-0.50)\end{array}$ & $\begin{array}{l}\text { moderate } \\
(\mathrm{WER}=1.85-1.95)\end{array}$ \\
\hline
\end{tabular}
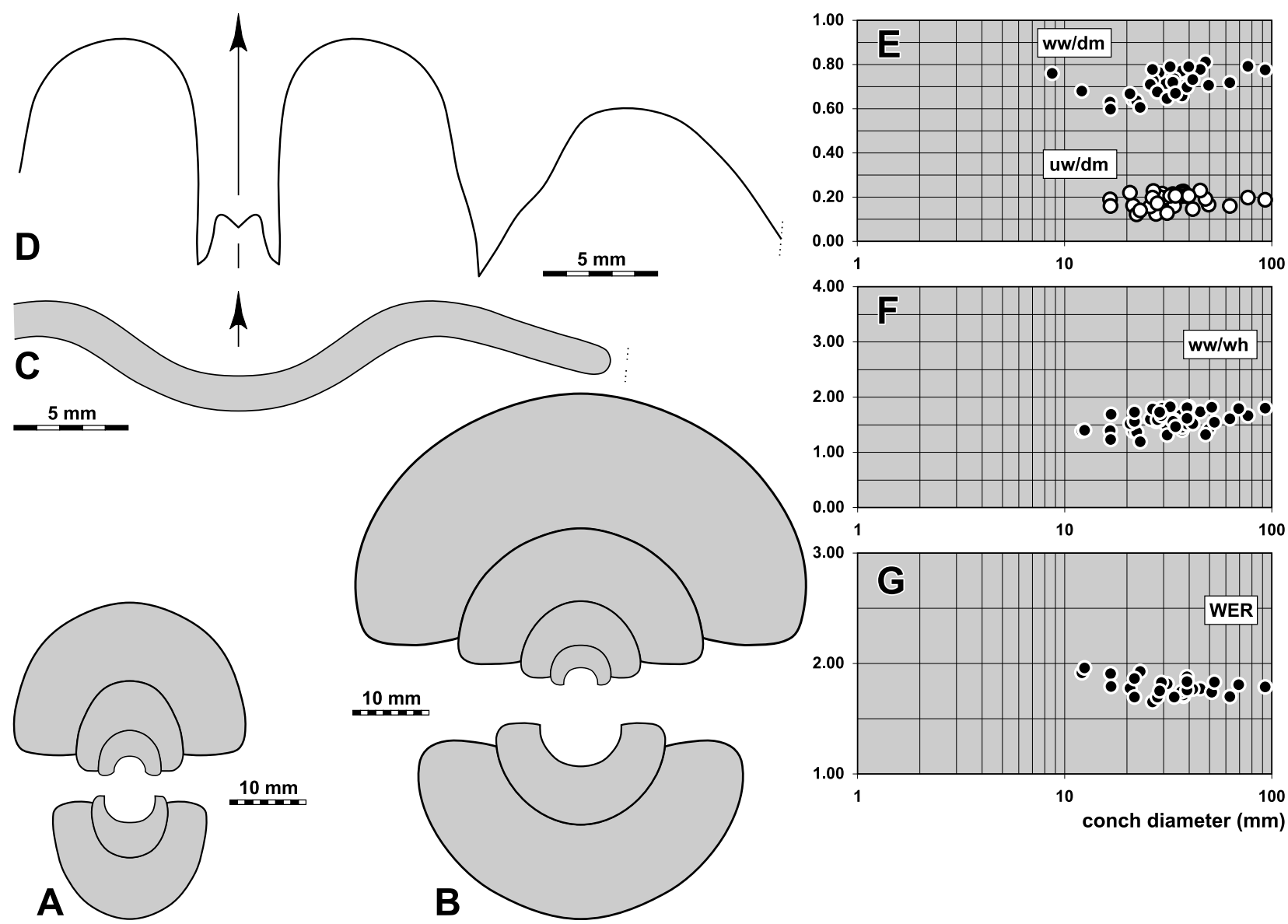

Figure 37. Obturgites polysarcus n. sp. from locality TIM-C7. A. Cross section of paratype MB.C.18613.6; $\times 1.0$. B. Cross section of paratype MB.C.18613.7; $\times 1.0$. C. Constriction of paratype MB.C.18613.8, at $20.6 \mathrm{~mm} w w, 11.6 \mathrm{~mm}$ wh; $\times 3.0$. D. Suture line of paratype MB.C.18613.4, at $33.3 \mathrm{~mm} \mathrm{dm}, 25.5 \mathrm{~mm}$ ww, $15.5 \mathrm{~mm}$ wh; $\times 3.0$. E-G. Ontogenetic development of the conch width index $(\mathrm{ww} / \mathrm{dm})$, umbilical width index (uw/dm), whorl width index (ww/wh), and whorl expansion rate (WER) of all available specimens. 
Table 37. Conch dimensions (in $\mathrm{mm}$ ) and proportions for reference specimens of Obturgites polysarcus $\mathrm{n}$. sp.

\begin{tabular}{|c|c|c|c|c|c|c|c|c|c|c|}
\hline & $\mathrm{dm}$ & ww & wh & uw & ah & $w w / d m$ & ww/wh & $\mathrm{uw} / \mathrm{dm}$ & WER & IZR \\
\hline paratype MB.C.18613.9 & 92.9 & 72.1 & 40.0 & 17.4 & 23.4 & 0.78 & 1.80 & 0.19 & 1.79 & 0.42 \\
\hline paratype MB.C.18613.2 & 62.6 & 45.0 & 28.0 & 10.0 & 14.6 & 0.72 & 1.61 & 0.16 & 1.70 & 0.48 \\
\hline paratype MB.C.18613.10 & 45.1 & 35.0 & 20.2 & 10.4 & 11.2 & 0.78 & 1.74 & 0.23 & 1.77 & 0.44 \\
\hline holotype MB.C.18613.1 & 39.7 & 31.4 & 17.2 & 8.2 & 9.9 & 0.79 & 1.82 & 0.21 & 1.77 & 0.43 \\
\hline paratype MB.C.18613.4 & 38.9 & 27.1 & 17.9 & 7.9 & 10.5 & 0.70 & 1.52 & 0.20 & 1.88 & 0.41 \\
\hline paratype MB.C. 18613.8 & 37.5 & 25.7 & 16.1 & 8.5 & 8.8 & 0.69 & 1.60 & 0.23 & 1.70 & 0.46 \\
\hline paratype MB.C.18613.5 & 36.6 & 28.2 & 16.8 & 7.5 & 8.9 & 0.77 & 1.68 & 0.20 & 1.74 & 0.47 \\
\hline paratype MB.C.18613.11 & 26.6 & 20.7 & 11.6 & 5.3 & 5.9 & 0.78 & 1.79 & 0.20 & 1.65 & 0.49 \\
\hline paratype MB.C.18613.12 & 20.7 & 13.8 & 9.1 & 4.6 & 5.2 & 0.67 & 1.52 & 0.22 & 1.78 & 0.43 \\
\hline
\end{tabular}

Table 38. Suture line proportions (Fig. 37D) for specimens of Obturgites polysarcus n. sp.

\begin{tabular}{llllll}
\hline specimen & at dm & EL w/d & EL/NLS & EL/AL & MS h \\
\hline paratype MB.C.18613.4 & $42.6 \mathrm{~mm}$ & 0.36 & 0.47 & 0.75 & 0.22 \\
\hline
\end{tabular}

Discussion. Obturgites polysarcus differs from the co-occurring species O. oligosarcus in the much stouter conch of specimens between 30 and $50 \mathrm{~mm} \mathrm{dm}(\mathrm{ww} / \mathrm{dm}=0.75-0.80$ in $O$. polysarcus but only $0.60-0.75$ in $O$. oligosarcus).

Species of the genus Eurites are superficially similar, but these possess a wider umbilicate intermediate growth stage and do not show the rapid increasing rate of the whorl width.

\title{
Obturgites oligosarcus n. sp.
}

\author{
Figures 35, 38, 39
}

Derivation of name. After Greek oligos = less and sarcus = flesh, because of the less voluminous body chamber.

Holotype. Specimen MB.C.18614.1, illustrated in Figure 38C.

Type locality and horizon. Sebkha de Timimoun, locality TIM-C7 (14.5 km west-southwest of Timimoun, Algeria); Ammonellipsites-Merocanites Assemblage.

Material. Ten specimens between 41 and $125 \mathrm{~mm}$ conch diameter are available. The largest of these is paratype MB.C.18614.5, it is still chambered at $125 \mathrm{~mm} \mathrm{dm}$, suggesting that the diameter with body chamber has been at least $220 \mathrm{~mm}$.

Diagnosis. Obturgites with a thickly pachyconic conch between 2 and $10 \mathrm{~mm} \mathrm{dm}$, thereafter lateral compression towards a thinly pachyconic conch at $15-60 \mathrm{~mm} \mathrm{dm}$; juvenile stage subinvolute, slight closure of the umbilicus in the adult stage; umbilical margin rounded or subangular; umbilical wall slightly flattened; aperture low or moderately high throughout ontogeny. Shell almost smooth; no constrictions on shell and steinkern. Suture line with very narrow, slightly pouched external lobe and low median saddle; ventrolateral saddle broadly rounded and asymmetric; adventive lobe asymmetric with almost straight ventral flank and strongly curved dorsal flank.

Table 39. Conch ontogeny (Figs 39A-C, E-G) of Obturgites oligosarcus n. sp.

\begin{tabular}{|c|c|c|c|}
\hline$d m$ & conch shape & whorl cross section shape & aperture \\
\hline $4 \mathrm{~mm}$ & $\begin{array}{l}\text { thickly pachyconic; subinvolute } \\
\text { (ww/dm } \sim 0.82 ; \text { uw/dm } \sim 0.20 \text { ) }\end{array}$ & $\begin{array}{l}\text { moderately depressed; strongly embracing } \\
\text { (ww/wh } \sim 1.85 ; \text { IZR } \sim 0.44 \text { ) }\end{array}$ & $\begin{array}{l}\text { moderate } \\
(\text { WER } \sim 1.95)\end{array}$ \\
\hline $10 \mathrm{~mm}$ & $\begin{array}{l}\text { thinly pachyconic; subinvolute } \\
\text { (ww/dm } \sim 0.70 ; u w / d m=0.16 \text { ) }\end{array}$ & $\begin{array}{l}\text { weakly depressed; strongly embracing } \\
\text { (ww/wh } \sim 1.35 ; \text { IZR } \sim 0.42 \text { ) }\end{array}$ & $\begin{array}{l}\text { moderate } \\
(\text { WER } \sim 1.95)\end{array}$ \\
\hline $30 \mathrm{~mm}$ & $\begin{array}{l}\text { thinly pachyconic; subinvolute } \\
(\mathrm{ww} / \mathrm{dm}=0.65-0.72 ; \mathrm{uw} / \mathrm{dm}=0.15-0.25)\end{array}$ & $\begin{array}{l}\text { moderately depressed; very strongly embracing } \\
\text { (ww/wh }=1.50-1.65 ; \text { IZR }=0.45-0.50)\end{array}$ & $\begin{array}{l}\text { moderate } \\
(\mathrm{WER}=1.75-1.85)\end{array}$ \\
\hline $60 \mathrm{~mm}$ & $\begin{array}{l}\text { thinly pachyconic; involute } \\
\text { (ww/dm } \sim 0.65 ; \mathrm{uw} / \mathrm{dm} \sim 0.10 \text { ) }\end{array}$ & $\begin{array}{l}\text { weakly depressed; strongly embracing } \\
\text { (ww/wh } \sim 1.15 ; \text { IZR } \sim 0.42 \text { ) }\end{array}$ & $\begin{array}{l}\text { high } \\
(\text { WER } \sim 2.10)\end{array}$ \\
\hline
\end{tabular}


Table 40. Conch dimensions (in $\mathrm{mm}$ ) and proportions for reference specimens of Obturgites oligosarcus n. sp.

\begin{tabular}{lrllllllllll}
\hline & \multicolumn{1}{c}{$\mathrm{dm}$} & ww & wh & uw & ah & ww/dm & ww/wh & uw/dm & WER & IZR \\
\hline paratype MB.C.18614.5 & 124.2 & 66.8 & 54.9 & 16.9 & 32.1 & 0.54 & 1.22 & 0.14 & 1.82 & 0.46 \\
paratype MB.C.18614.2 & 51.6 & 32.1 & 28.5 & 6.8 & 14.9 & 0.62 & 1.12 & 0.13 & 1.97 & 0.48 \\
holotype MB.C.18614.1 & 44.5 & 30.0 & 22.3 & 6.7 & 11.5 & 0.68 & 1.35 & 0.15 & 1.82 & 0.48 \\
paratype MB.C.18614.3 & 41.7 & 28.4 & 22.4 & 6.0 & 12.4 & 0.68 & 1.27 & 0.14 & 2.02 & 0.45 \\
\hline
\end{tabular}

Table 41. Suture line proportions (Fig. 39D) for specimens of Obturgites oligosarcus n. sp.

\begin{tabular}{lllllll}
\hline specimen & at $\mathrm{dm}$ & EL w/d & EL/VLS & EL/AL & MS h & VLS w/h \\
\hline holotype MB.C.18614.1 & $42.6 \mathrm{~mm}$ & 0.38 & 0.56 & 0.85 & 0.20 & 0.67 \\
\hline
\end{tabular}

Discussion. Obturgites oligosarcus differs from the co-occurring species O.polysarcus in the much more slender conch $(\mathrm{ww} / \mathrm{dm}=0.75-0.80$ in $O$. polysarcus but only $0.60-0.75$ in $O$. oligosarcus at $30-50 \mathrm{~mm} \mathrm{dm}$ ).
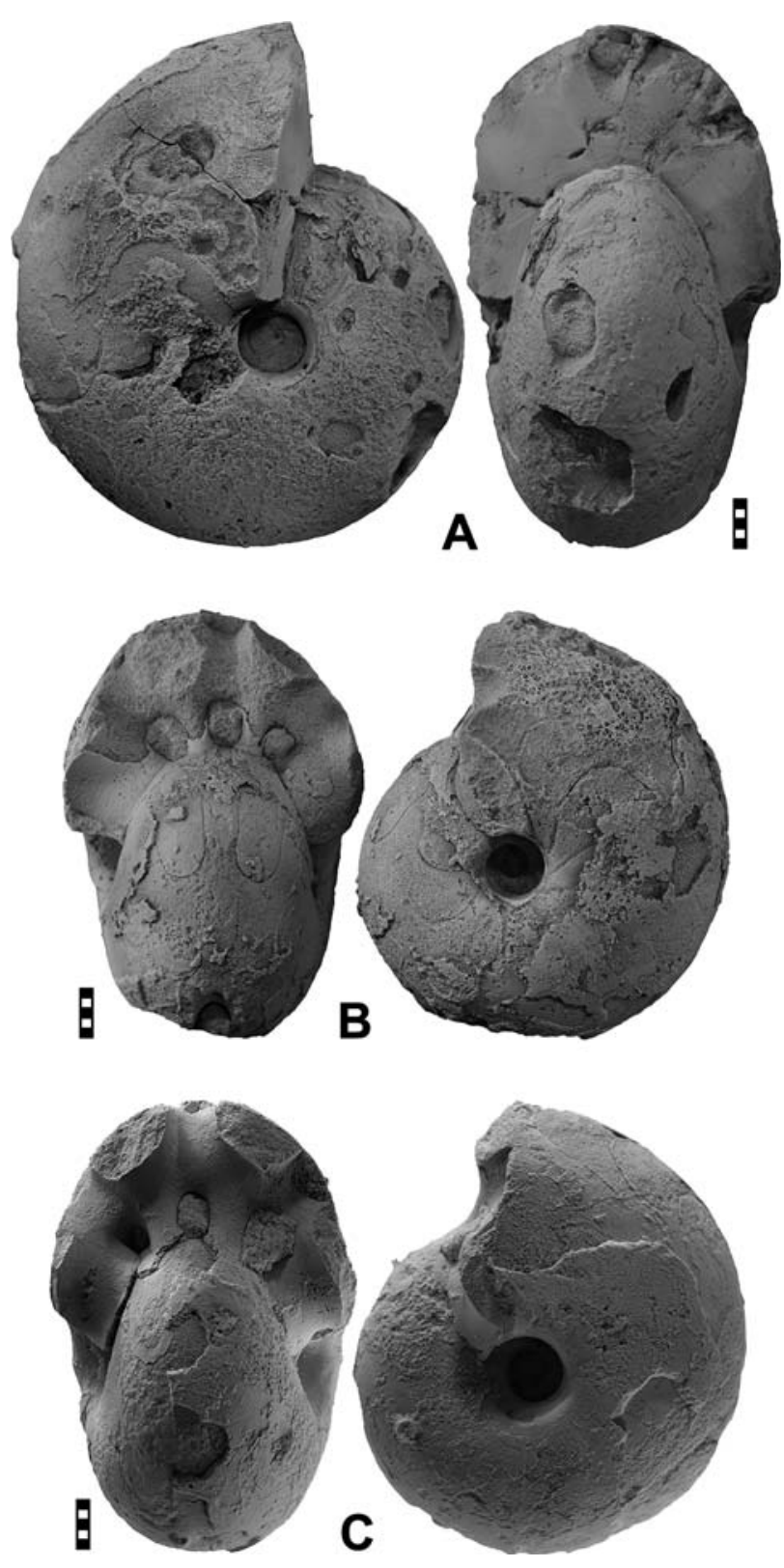

Figure 38. Obturgites oligosarcus n. sp. from locality TIMC7; all $\times 1.0$. A. Paratype MB.C.18614.2. B. Paratype MB.C.18614.3. C. Holotype MB.C.18614.1. 

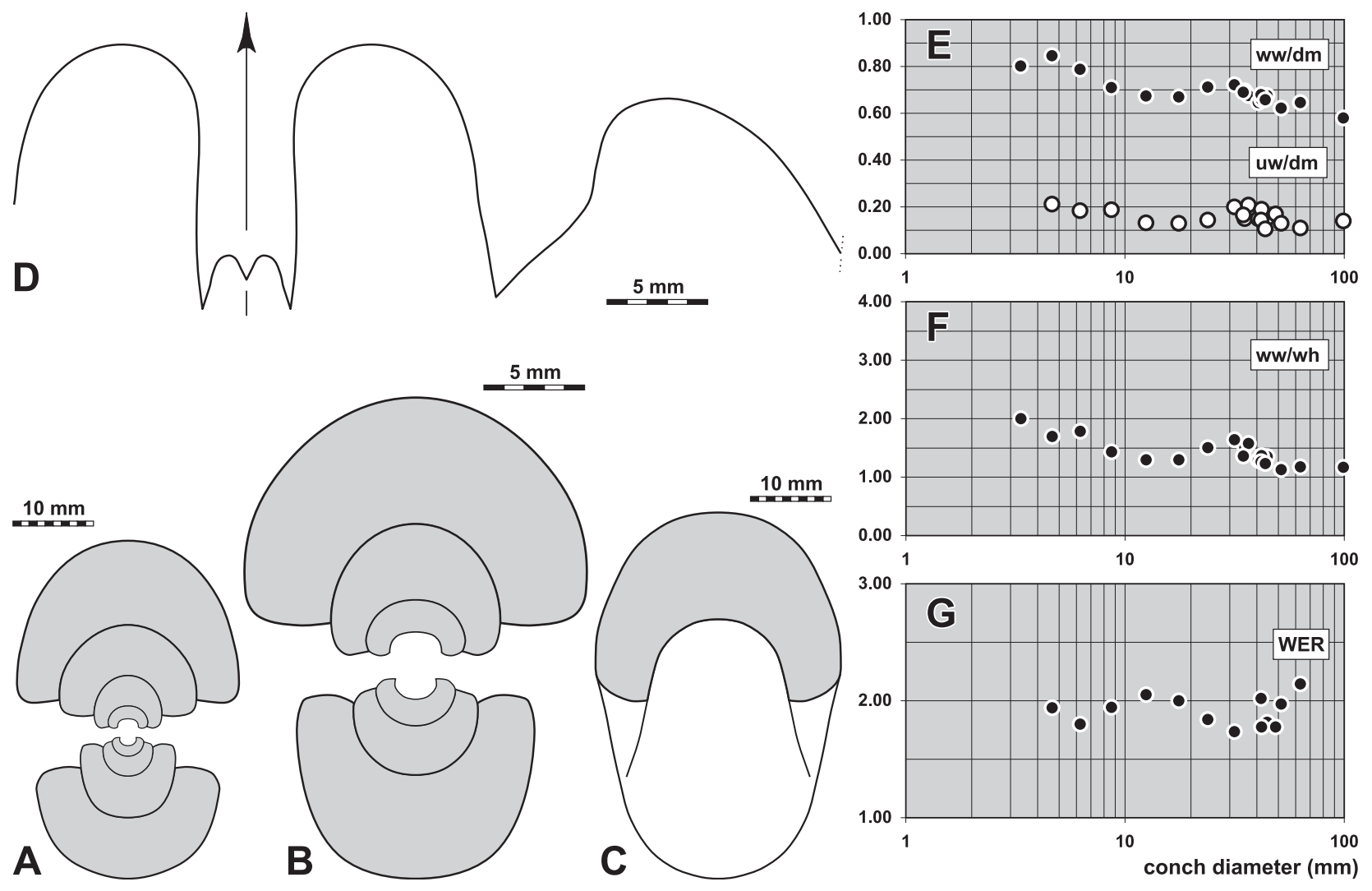

Figure 39. Obturgites oligosarcus n. sp. from locality TIM-C7. A. Cross section of paratype MB.C.18614.4; $\times 1.0$. B. Cross section (inner whorls) of the same specimen; $\times 2.5$. C. Dorsal view of holotype MB.C.18614.1; $\times 1.0$. D. Suture line of holotype MB.C.18614.1, at $42.6 \mathrm{~mm} \mathrm{dm}, 29.0 \mathrm{~mm}$ ww, $20.3 \mathrm{~mm} \mathrm{wh;} \times 2.5$. E-G. Ontogenetic development of the conch width index (ww/dm), umbilical width index (uw/dm), whorl width index (ww/wh), and whorl expansion rate (WER) of all available specimens.

Family Maxigoniatitidae Korn, Klug \& Mapes, 1999

\section{Dzhaprakoceras Popov, 1965}

Type species. Muensteroceras tianshanicum Librovitch, 1927 (OD).

Genus definition. Maxigoniatitidae with insignificant ontogenetic changes in the conch; shape pachyconic or discoidal, involute or subinvolute with an ontogenetic tendency for lateral compression of the conch and closure of the umbilicus (Fig. 40); flanks moderately strong converging towards the rounded venter; umbilical wall and umbilical margin rounded; aperture high in the adult stage. Ornament with very fine, biconvex rectiradiate growth lines with moderately ventrolateral projection and rather deep ventral sinus; sometimes with shell constrictions parallel to the growth lines. Suture line with very narrow, slightly pouched external lobe and very low or low median saddle; ventrolateral saddle broadly rounded and usually strikingly asymmetric; adventive lobe V-shaped, usually asymmetric with almost straight or concave ventral side and convex dorsal side.

Included species.

aksuense: Muensteroceratoides aksuensis Popov, 1965, p. 37; Tien Shan.

angustum: Dzhaprakoceras angustum Kusina \& Konovalova, 2004, p. 15; North Urals.

arkansanum: Muensteroceras arkansanum Gordon, 1965, p. 177; Arkansas.

belcourtense: Dzhaprakoceras belcourtense Work \& Nassichuk, 2000, p. 44; British Columbia.

catena: Dzhaprakoceras catena Riley, 1996, p. 53; Lancashire.

chermnykhi: Dzhaprakoceras chermnykhi Kusina, 1980, p. 51; North Urals.

crassum: Dzhaprakoceras crassum Work \& Nassichuk, 2000, p. 45; British Columbia.

deflexum: Dzhaprakoceras deflexum Kusina, 1980, p. 58; North Urals.

djaprakense: Muensteroceras djaprakense Librovitch, 1927, p. 37; Tien Shan.

duponti: Munsteroceras duponti Delépine, 1940, p. 58; Belgium.

flexiforme: Dzhaprakoceras flexiforme Kusina \& Konovalova, 2004, p. 15; North Urals.

flexum: Dzhaprakoceras flexum Riley, 1996, p. 58; Lancashire.

flexuosum: Nautellipsites pseudoparallelus forma flexuosa Pareyn, 1961, p. 106; Saoura Valley. [synonym of D. hispanicum] glabrum: Dzhaprakoceras glabrum Kusina \& Konovalova, 2004, p. 13; North Urals.

gordoni: Dzhaprakoceras gordoni Petersen, Korn \& Kullmann, 2000, p. 885; Utah.

gracile: Dzhaprakoceras gracile Kusina in Shimansky \& Kusina, 1977, p. 87; North Urals. 
hibernicum: Nautellipsites hibernicus Delépine, 1940, p. 164; Ireland.

hispanicum: Glyphioceras hispanicum Foord \& Crick, 1903, p. 190; Cantabrian Mountains.

humile: Münsteroceras humile Schindewolf, 1951, p. 62; Harz.

improcerum: Dzhaprakoceras improcerum Kusina, 1980, p. 50; North Urals.

latilobatum: Dzhaprakoceras latilobatum Kusina, 1983, p. 93; North Urals.

levis: Dzhaprakoceras levis Riley, 1996, p. 61; Lancashire.

narynense: Bollandites narynensis Popov, 1965, p. 39; Tien Shan.

paracatena: Dzhaprakoceras paracatena Riley, 1996, p. 63; Lancashire.

planum: Dzhaprakoceras planum Kusina \& Yatskov, 1987, p. 103; Novaya Zemlya.

procerum: Münsteroceras procerum Schindewolf, 1951, p. 57; Harz.

pseudoparallelum: Nautellipsites pseudoparallelum Delépine, 1940, p. 54; Anti-Atlas.

quantulum: Dzhaprakoceras quantulum Kusina \& Yatskov, 1987, p. 104; Novaya Zemlya.

shabyrense: Bollandites shabyrensis Popov, 1968, p. 93; Tien Shan.

sonkulicum: Muensteroceras kazahstanicum var. sonkulica Librovitch, 1940, p. 32; Karaganda.

subglobosum: Muensteroceras subglobosum Librovitch, 1927, p. 35; Tien Shan.

subquadratum: Glyphioceras (Beyrichoceras) subquadratum Foord, 1903, p. 172; Ireland.

subtruncatum: Glyphioceras (Beyrichoceras) subtruncatum Foord, 1903, p. 168; Ireland.

subtile: Dzhaprakoceras subtile Kusina, 1980, p. 54; North Urals.

tianshanicum: Muensteroceras tianshanicum Librovitch, 1927, p. 33; Tien Shan.

? difficile: Glyphioceras (Beyrichoceras) difficile Foord, 1903, p. 171; Ireland.

? kokdzharensis: ?Bollandites kokdzharensis Popov, 1965, p. 41; Tien Shan.

dzhazairense: Dzhaprakoceras dzhazairense n. sp.; Gourara, Algeria.
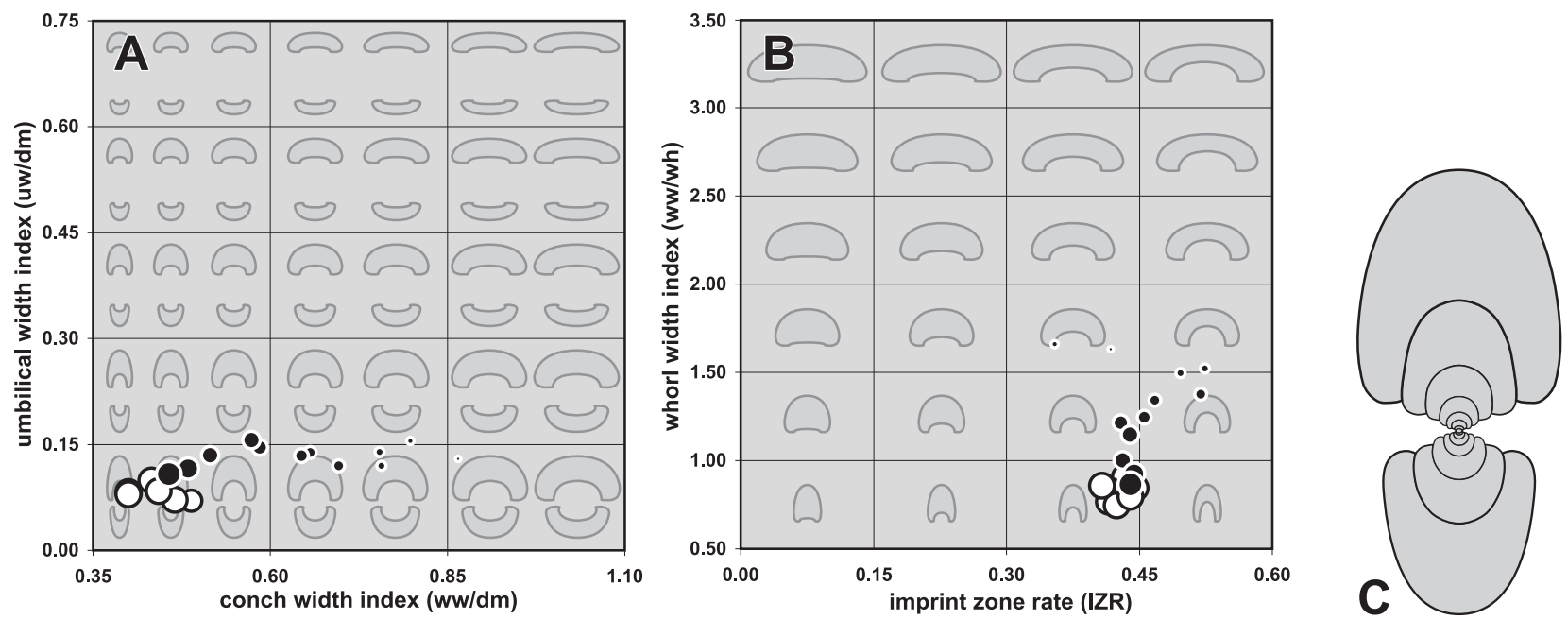

Figure 40. Ontogenetic trajectories of Dzhaprakoceras, exemplified for Dzhaprakoceras dzhazairense n. sp. from locality TIM-C7. A. Ontogenetic development of the conch width index (ww/dm), umbilical width index (uw/dm). B. Ontogenetic development of the imprint zone rate (IZR), whorl width index (ww/wh). C. Cross section of paratype MB.C.18615.12; $\times 1.0$. [Black dots represent ontogenetic stages of cross section MB.C.18615.12, white dots represent the reference specimens (Tab. 43).]

\section{Dzhaprakoceras dzhazairense n. sp.}

Figures 41, 42

Derivation of name. After Al Dzhazair, the Arab name for Algeria.

Holotype. Specimen MB.C.18615.1, illustrated in Figure 41A.

Type locality and horizon. Sebkha de Timimoun, locality TIM-C7 (14.5 km west-southwest of Timimoun, Algeria); Ammonellipsites-Merocanites Assemblage.

Material. The species is the most common in the Dalle à Merocanites and is represented by well-preserved material; a total of 98 specimens between 25 and $87 \mathrm{~mm}$ conch diameter were studied. The maximum diameter of the specimens with body chamber can be estimated to be within about $120 \mathrm{~mm}$.

Diagnosis. Dzhaprakoceras with an involute conch throughout ontogeny, shape continuously transformed from thickly pachyconic at $2 \mathrm{~mm} \mathrm{dm}$ to thinly discoidal at $60 \mathrm{~mm} \mathrm{dm}$; umbilical margin rounded in all growth stages; aperture moderately high in early growth stages and slightly increasing in the adult stage. Ornament with very fine biconvex growth lines, course biconvex with very low lateral sinus and deep ventral sinus, direction rectiradiate; shell and steinkern without constrictions. Suture line with very narrow, almost parallel-sided and slightly pouched external lobe and low median saddle; ventrolateral saddle strongly asymmetric and broadly rounded; adventive lobe V-shaped, usually strongly asymmetric with strongly convex dorsal flank. 
Table 42. Conch ontogeny (Figs 42A-I, N-P) of Dzhaprakoceras dzhazairense n. sp.

\begin{tabular}{|c|c|c|c|}
\hline$d m$ & conch shape & whorl cross section shape & aperture \\
\hline $4 \mathrm{~mm}$ & $\begin{array}{l}\text { thinly pachyconic; involute } \\
(\mathrm{ww} / \mathrm{dm}=0.65-0.70 ; \mathrm{uw} / \mathrm{dm}=0.10-0.15)\end{array}$ & $\begin{array}{l}\text { weakly depressed; very strongly embracing } \\
\text { (ww/wh }=1.30-1.50 ; \text { IZR }=0.45-0.52)\end{array}$ & $\begin{array}{l}\text { moderate } \\
(\mathrm{WER}=1.75-1.95)\end{array}$ \\
\hline $10 \mathrm{~mm}$ & $\begin{array}{l}\text { thickly discoidal; involute } \\
(\mathrm{ww} / \mathrm{dm}=0.55-0.60 ; \mathrm{uw} / \mathrm{dm}=0.07-0.15)\end{array}$ & $\begin{array}{l}\text { weakly depressed; strongly embracing } \\
(\text { ww/wh }=1.00-1.25 ; \text { IZR }=0.40-0.45)\end{array}$ & $\begin{array}{l}\text { moderate to high } \\
(\mathrm{WER}=1.95-2.15)\end{array}$ \\
\hline $30 \mathrm{~mm}$ & $\begin{array}{l}\text { thinly to thickly discoidal; involute } \\
\text { (ww/dm }=0.45-0.55 ; u w / d m=0.07-0.15 \text { ) }\end{array}$ & $\begin{array}{l}\text { weakly compressed to weakly depressed; strongly } \\
\text { embracing (ww/wh }=0.90-1.15 ; \text { IZR }=0.40-0.45 \text { ) }\end{array}$ & $\begin{array}{l}\text { moderate to high } \\
(\mathrm{WER}=1.95-2.25)\end{array}$ \\
\hline $60 \mathrm{~mm}$ & $\begin{array}{l}\text { thinly discoidal; involute } \\
(\mathrm{ww} / \mathrm{dm}=0.40-0.45 ; \mathrm{uw} / \mathrm{dm}=0.05-0.10 \text { ) }\end{array}$ & $\begin{array}{l}\text { weakly compressed; strongly embracing } \\
(\mathrm{ww} / \mathrm{wh}=0.75-0.85 ; \mathrm{IZR}=0.40-0.45)\end{array}$ & $\begin{array}{l}\text { moderate to high } \\
\text { (WER }=1.95-2.20)\end{array}$ \\
\hline
\end{tabular}

Table 43. Conch dimensions (in $\mathrm{mm}$ ) and proportions for reference specimens of Dzhaprakoceras dzhazairense $\mathrm{n}$. sp.

\begin{tabular}{lllllllllll}
\hline & dm & ww & wh & uw & ah & ww/dm & ww/wh & uw/dm & WER & IZR \\
\hline paratype MB.C.18615.15 & 56.6 & 22.7 & 28.9 & 4.6 & 16.5 & 0.40 & 0.78 & 0.08 & 1.99 & 0.43 \\
paratype MB.C.18615.16 & 55.1 & 24.5 & 29.5 & 5.2 & 17.2 & 0.45 & 0.83 & 0.09 & 2.12 & 0.42 \\
holotype MB.C.18615.1 & 52.9 & 21.1 & 27.4 & 4.4 & 16.0 & 0.40 & 0.77 & 0.08 & 2.06 & 0.42 \\
paratype MB.C.18615.17 & 46.5 & 21.7 & 25.5 & 3.3 & 14.1 & 0.47 & 0.85 & 0.07 & 2.06 & 0.45 \\
paratype MB.C.18615.3 & 45.9 & 18.3 & 24.4 & 3.6 & 14.1 & 0.40 & 0.75 & 0.08 & 2.08 & 0.42 \\
paratype MB.C.18615.4 & 44.4 & 19.2 & 24.0 & 4.4 & 13.5 & 0.43 & 0.80 & 0.10 & 2.06 & 0.44 \\
paratype MB.C.18615.2 & 42.3 & 18.7 & 21.7 & 3.6 & 12.9 & 0.44 & 0.86 & 0.08 & 2.06 & 0.41 \\
paratype MB.C.18615.18 & 32.7 & 16.0 & 17.5 & 2.3 & 9.9 & 0.49 & 0.91 & 0.07 & 2.06 & 0.43 \\
\hline
\end{tabular}

Table 44. Suture line proportions (Figs 42J-M) for Dzhaprakoceras dzhazairense n. sp.

\begin{tabular}{|c|c|c|c|c|c|c|c|}
\hline specimen & at $d m$ & $E L w / d$ & EL/NLS & $\mathrm{EL} / \mathrm{AL}$ & MS h & VLS w/h & remarks \\
\hline paratype MB.C.18615.14 & $54.0 \mathrm{~mm}$ & 0.31 & 0.52 & 1.01 & 0.25 & 0.60 & A lobe strongly asymmetric \\
\hline paratype MB.C.18615.16 & c. $45.0 \mathrm{~mm}$ & 0.39 & 0.58 & 0.91 & 0.22 & 0.67 & \\
\hline holotype MB.C.18615.1 & $40.4 \mathrm{~mm}$ & 0.39 & 0.48 & 0.71 & 0.27 & 0.80 & A lobe almost symmetric \\
\hline paratype MB.C.18615.13 & c. $38.0 \mathrm{~mm}$ & 0.35 & 0.45 & 0.87 & 0.21 & 0.79 & VL saddle almost symmetric \\
\hline paratype MB.C.18615.4 & c. $27.0 \mathrm{~mm}$ & 0.41 & 0.63 & 0.92 & 0.24 & 0.64 & \\
\hline
\end{tabular}

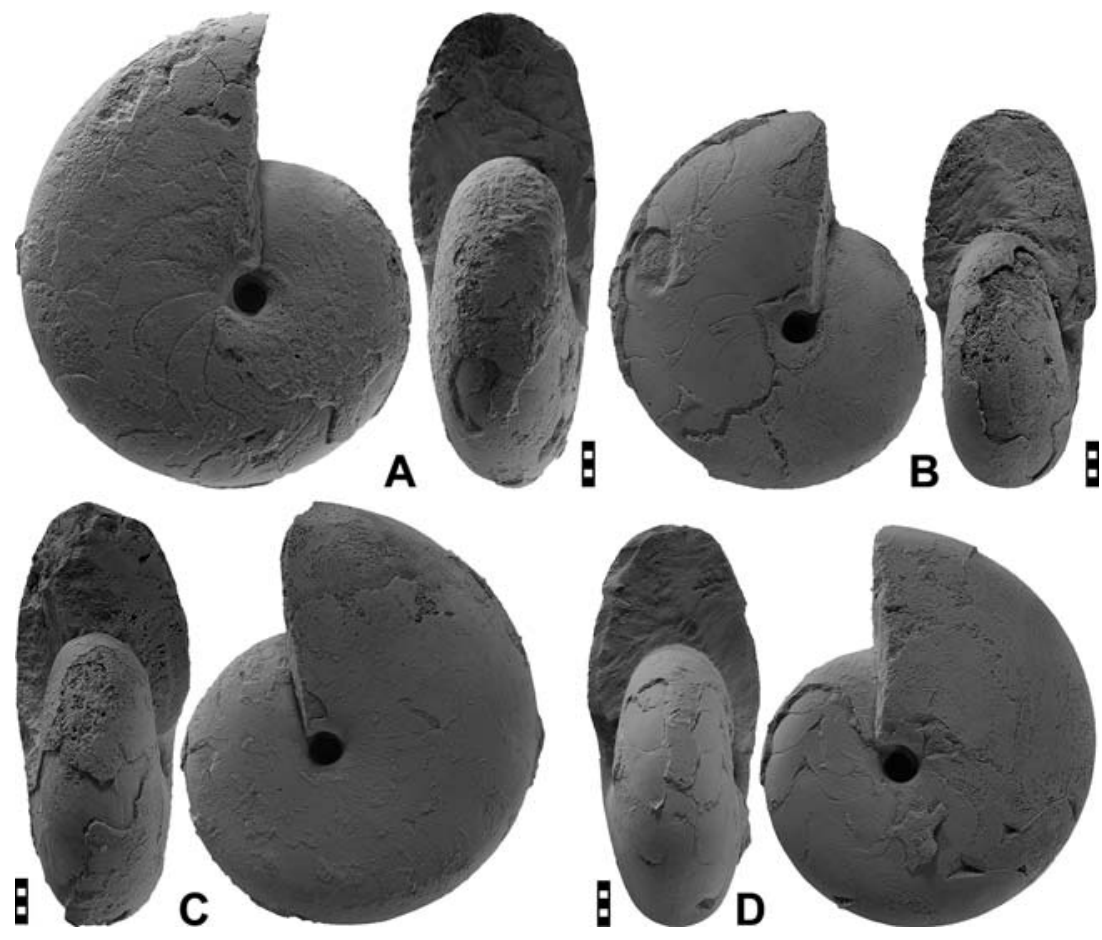

Figure 41. Dzhaprakoceras dzhazairense n. sp. from locality TIM-C7; all $\times 1.0$. A. Holotype MB.C.18615.1. B. Paratype MB.C.18615.2. C. Paratype MB.C.18615.3. D. Paratype MB.C. 18615.4 
Discussion. Dzhaprakoceras dzhazairense belongs to the slender and involute species of the genus. It differs from the co-occurring Muensteroceras fabrei in the ww/dm ratio that decreases continuously in D. dzhazairense but shows an adult stabilization in M. fabrei. Furthermore, the aperture in D. dzhazairense becomes almost continuously higher, whereas it stagnates at 1.90-2.10 in adult conchs of $M$. fabrei. The external lobe of M. fabrei has almost parallel flanks.

The type species of Dzhaprakoceras, D. tianshanicum (Librovitch, 1927), has a similar conch like D. dzhazairense, but differs in the wider external lobe and the symmetric adventive lobe. According to the cross section illustrated by Popov (1968, fig. 15b), the inner whorls are more widely umbilicate than in D. dzhazairense (uw/ $\mathrm{dm}=0.27$ at $4.5 \mathrm{~mm} \mathrm{dm}$ in D. tianshanicum and only $0.12-0.15$ in D. dzhazairense).

D. chermnykhi Kusina, 1980 is similar to D. dzhazairense, but that species has an almost closed umbilicus (uw/ $\mathrm{dm}=0.06$ at $43 \mathrm{~mm} \mathrm{dm}$ ) and a much wider external lobe. D. subtile Kusina, 1980 has a similar conch but possesses a wider external lobe and a symmetric adventive lobe. D. gracile Kusina, 1977 has a similar conch and
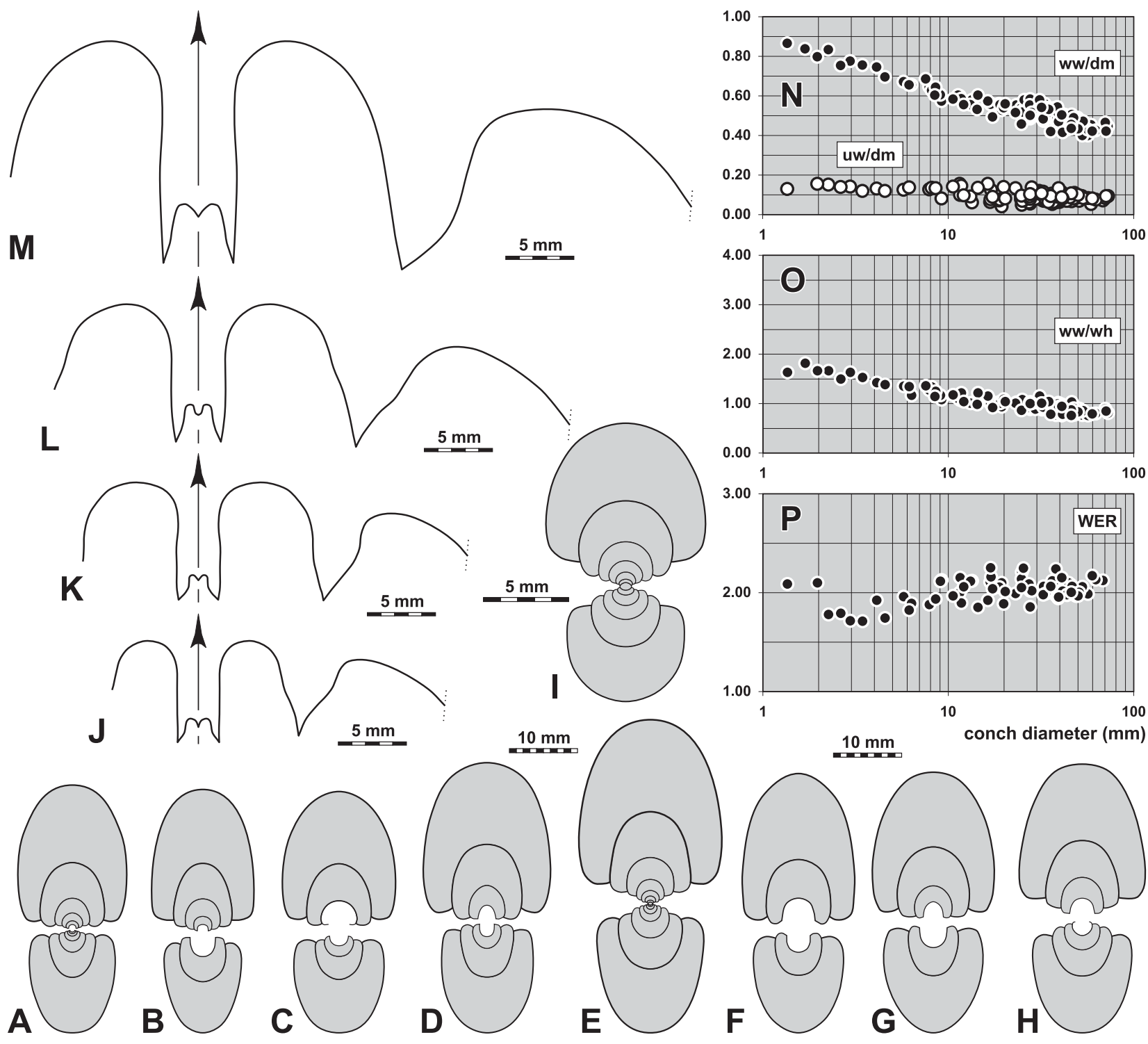

Figure 42. Dzhaprakoceras dzhazairense n. sp. from locality TIM-C7. A. Cross section of paratype MB.C.18615.5; $\times 1.0$. B. Cross section of paratype MB.C.18615.6; $\times 1.0$. C. Cross section of paratype MB.C.18615.7; $\times 1.0$. D. Cross section of paratype MB.C.18615.8; $\times 1.0$. E. Cross section of paratype MB.C.18615.12; $\times 1.0$. F. Cross section of paratype MB.C.18615.9; $\times 1.0$. G. Cross section of paratype MB.C.18615.10; $\times 1.0$. H. Cross section of paratype MB.C.18615.11; $\times 1.0$. I. Cross section (inner whorls) of paratype MB.C.18615.12; $\times 2.5$. J. Suture line of paratype MB.C.18615.4, at $13.3 \mathrm{~mm} w w, 14.8 \mathrm{~mm}$ wh; $\times 2.0$. K. Suture line of paratype MB.C.18615.13, at $17.8 \mathrm{~mm} \mathrm{dm}, 13.7 \mathrm{~mm}$ ww, $17.2 \mathrm{~mm}$ wh; $\times 2.0$. L. Suture line of paratype MB.C.18615.1, at $40.4 \mathrm{~mm} \mathrm{dm}, 18.5 \mathrm{~mm}$ ww, $21.2 \mathrm{~mm}$ wh; $\times 2.0$. M. Suture line of paratype MB.C.18615.14, at $54.0 \mathrm{~mm} \mathrm{dm,} 24.4 \mathrm{~mm}$ ww, $28.3 \mathrm{~mm}$ wh; $\times 2.0$. N-P. Ontogenetic development of the conch width index (ww/dm), umbilical width index (uw/dm), whorl width index (ww/wh), and whorl expansion rate (WER) of all available specimens. 
suture line, but is more slender than D. dzhazairense (ww/dm $=0.36$ in D. gracile at $45 \mathrm{~mm} \mathrm{dm}$ but $0.45-0.50$ in D. dzhazairense).

D. hispanicum (Foord \& Crick, 1897) is difficult to compare because it is based on a single corroded and fully septate specimen (NHM 37492a) of $54 \mathrm{~mm}$ conch diameter from the Cantabrian Mountains. The conch is thinly discoidal $(\mathrm{ww} / \mathrm{dm}=0.41)$ and involute $(\mathrm{uw} / \mathrm{dm}=0.12)$. The specimen has a parallel-sided external lobe, but this may be an effect of corrosion. D. hibernicum (Delépine, 1940) is based on a large specimen (NHM c38180, J. Sowerby Coll.; $104 \mathrm{~mm} \mathrm{dm})$ from an unknown locality in Ireland. It is thinly discoidal $(\mathrm{ww} / \mathrm{dm}=0.34)$ and involute $(\mathrm{uw} / \mathrm{dm}=0.07)$; in this it resembles the conch of larger specimens of D. dzhazairense. The suture line of the specimen also shows a parallel-sided external lobe, but in this case a possible sinuosity of the external lobe flanks may be an artefact of deep grinding. The suture line of D. hispanicum and also that of D. hibernicum have a rather symmetric adventive lobe and thus differ markedly from $D$. dzhazairense.

"Nautellipsites pseudoparallelus Delépine, 1941" from Erfoud (Anti-Atlas, Morocco) may also belong to Dzhaprakoceras, but this species is known from only one specimen (the others listed by Delépine came from Timimoun) of $17 \mathrm{~mm} \mathrm{dm}$. The umbilicus is nearly closed in this specimen. The specimens from Djebel Ioucha (Saoura Valley, Algeria) referred by Pareyn (1961, p. 106) to this species and the "forma flexuosa" clearly belong to Dzhaprakoceras. They have a very low median saddle and differ in this respect from $D$. dzhazairense.

Order Prolecanitida Miller \& Furnish, 1954

Suborder Prolecanitina Miller \& Furnish, 1954

Superfamily Prolecanitaceae Hyatt, 1884

Family Prolecanitidae Hyatt, 1884

Subfamily Protocanitinae Weyer, 1972

\section{Merocanites Schindewolf, 1922}

Type species. Ellipsolithes compressus Sowerby, 1813 (OD).

Included species.

applanatus: Prolecanites applanatus Frech, 1899, p. pl. 46a; Rhenish Mountains.

compressus: Ellipsolithes compressus Sowerby, 1813, p. 37; Ireland.

djaprakensis: Prolecanites (Merocanites) djaprakensis Librovitch, 1927, p. 38; Tien Shan.

drostei: Merocanites drostei Collinson, 1955, p. 434; Kentucky.

henslowi: Ammonites Henslowi Sowerby, 1820, p. 111; Ireland.

holzapfeli: Prolecanites Holzapfeli Frech, 1902, p. 66; Rhenish Mountains. [Synonym of M. applanatus]

houghtoni: Goniatites Houghtoni Winchell, 1862, p. 363; Michigan.

ogivalis: Merocanites ogivalis Pareyn, 1961, p. 82; South Oran, Algeria.

planorbis: Merocanites planorbis Delépine, 1941, p. 44; Morocco.

rileyi: Merocanites rileyi Work \& Nassichuk, 2000, p. 29; British Columbia.

similis: Prolecanites similis Crick, 1895, p. 180; Yorkshire.

subapplanatus: Merocanites subapplanatus Smyth, 1951, p. 290; Ireland.

subhenslowi: Merocanites subhenslowi Wagner-Gentis, 1964, p. 238; Cantabrian Mountains. [Synonym of M. applanatus]

tenuis: Merocanites tenuis Sheng, 1984, p. 287; Xinjiang.

quadrilobus: Merocanites quadrilobus Riley, 1996, p. 18; Lancashire.

\section{Merocanites merocanites $\mathbf{n}$. sp.}

Figures 43, 44

1984 Merocanites applanatus. - Conrad, pl. 5, fig. 7.

Derivation of name. Tautonym of the genus name.

Holotype. Specimen MB.C.18616.1, illustrated in Figure 43A.

Type locality and horizon. Sebkha de Timimoun, locality TIM-C7 (14.5 km west-southwest of Timimoun, Algeria); Ammonellipsites-Merocanites Assemblage.

Material. Fourteen moderately preserved specimens between 16 and $66 \mathrm{~mm}$ conch diameter are available for study.

Diagnosis. Merocanites with strongly applanate whorl cross section (ww/wh $=0.62$ at $65 \mathrm{~mm} \mathrm{dm}$ ); flattened and subparallel and separated from slightly flattened venter by a subangular ventrolateral edge; umbilical margin rounded. Shell surface almost smooth. Suture line with small Vshaped external lobe; three lobes present on the flanks, the outer two of these resemble each other in their strikingly asymmetric form with a steeper ventral flank and a prominently curved dorsal flank, they are separated by an inflated saddle.

Discussion. Many of the species of Merocanites require revision and are thus very difficult to compare with the new species. M. merocanites differs from M. applanatus, M. djaprakensis, M. ogivalis, and M. quadrilobus in the strongly asymmetric shape of the outer two lobes on the flank (adventive lobe and first lateral lobe). 
Table 45. Conch ontogeny (Figs 44A, C-E) of Merocanites merocanites n. sp.

\begin{tabular}{|c|c|c|c|}
\hline$d m$ & conch shape & whorl cross section shape & aperture \\
\hline $16 \mathrm{~mm}$ & $\begin{array}{l}\text { extremely discoidal; evolute } \\
(\mathrm{ww} / \mathrm{dm} \sim 0.25 ; \mathrm{uw} / \mathrm{dm} \sim 0.45)\end{array}$ & $\begin{array}{l}\text { weakly compressed; weakly embracing } \\
\text { (ww/wh } \sim 0.80 ; \text { IZR } \sim 0.05 \text { ) }\end{array}$ & $\begin{array}{l}\text { moderate to high } \\
(\mathrm{WER} \sim 2.00)\end{array}$ \\
\hline $30 \mathrm{~mm}$ & $\begin{array}{l}\text { extremely discoidal; evolute } \\
(\mathrm{ww} / \mathrm{dm}=0.24-0.30 ; \mathrm{uw} / \mathrm{dm}=0.45-0.50)\end{array}$ & $\begin{array}{l}\text { weakly compressed; weakly embracing } \\
\text { (ww/wh }=0.75-0.90 ; \text { IZR }=0.05-0.08)\end{array}$ & $\begin{array}{l}\text { moderate to high } \\
\text { (WER }=1.95-2.10)\end{array}$ \\
\hline $60 \mathrm{~mm}$ & $\begin{array}{l}\text { extremely discoidal; evolute } \\
(\mathrm{ww} / \mathrm{dm}=0.18-0.22 ; \mathrm{uw} / \mathrm{dm}=0.45-0.50)\end{array}$ & $\begin{array}{l}\text { weakly compressed; weakly embracing } \\
(\text { ww/wh }=0.60-0.70 ; \text { IZR }=0.05-0.08)\end{array}$ & $\begin{array}{l}\text { moderate to high } \\
(\text { WER }=1.95-2.10)\end{array}$ \\
\hline
\end{tabular}

Table 46. Conch dimensions (in $\mathrm{mm}$ ) and proportions for reference specimens of Merocanites merocanites $\mathrm{n}$. sp.

\begin{tabular}{|c|c|c|c|c|c|c|c|c|c|c|}
\hline & $\mathrm{dm}$ & ww & wh & uw & ah & $w w / d m$ & ww/wh & $\mathrm{uw} / \mathrm{dm}$ & WER & IZR \\
\hline holotype MB.C.18616.1 & 65.6 & 12.7 & 20.6 & 31.0 & 19.3 & 0.19 & 0.62 & 0.47 & 2.01 & 0.06 \\
\hline paratype MB.C.18616.2 & 60.8 & 12.5 & 18.9 & 29.5 & 17.7 & 0.21 & 0.66 & 0.48 & 1.99 & 0.06 \\
\hline paratype MB.C.18616.3 & 43.8 & 10.5 & 14.3 & 19.5 & 13.6 & 0.24 & 0.73 & 0.45 & 2.10 & 0.05 \\
\hline paratype MB.C.18616.4 & 29.0 & 6.5 & 8.7 & 14.1 & 7.9 & 0.22 & 0.74 & 0.49 & 1.88 & 0.10 \\
\hline paratype MB.C.18616.5 & 16.0 & 4.1 & 5.1 & 7.4 & 4.9 & 0.25 & 0.80 & 0.46 & 2.02 & 0.06 \\
\hline
\end{tabular}
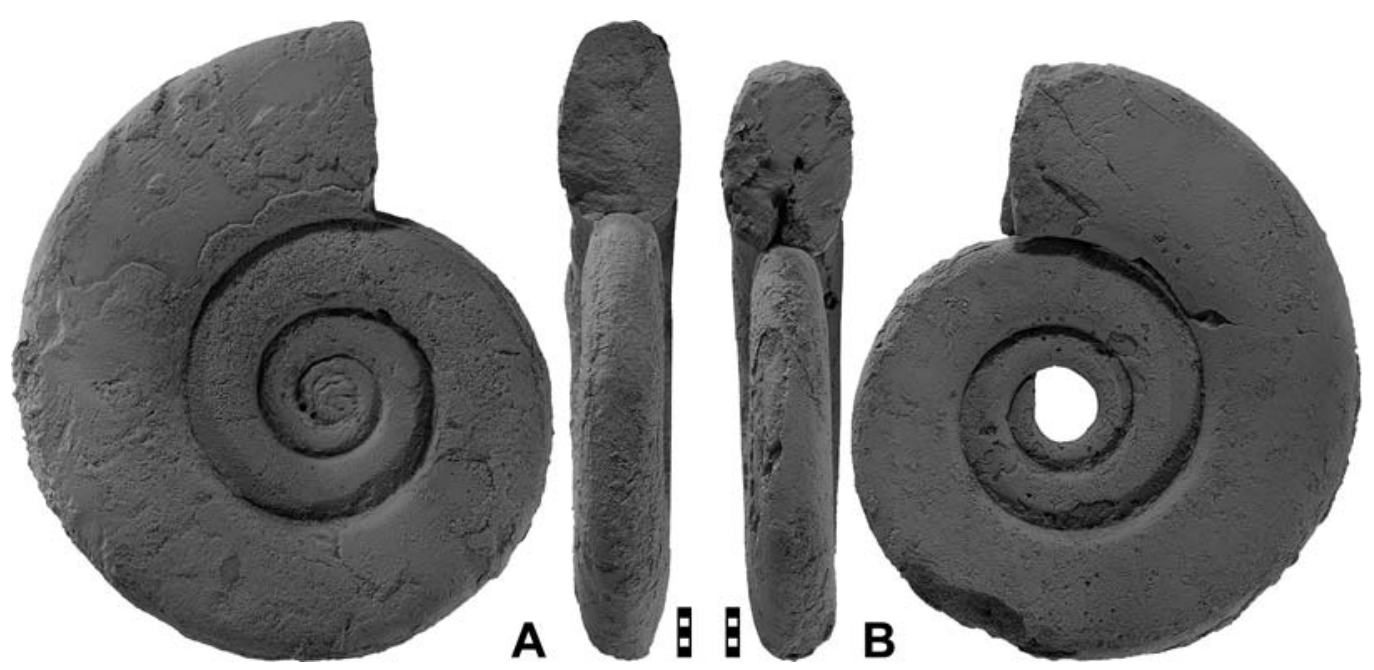

Figure 43. Merocanites merocanites n. sp. from locality TIM-C7. A. Holotype MB.C.18616.1;×1.0. B. Paratype MB.C.18616.2; × 1.0.
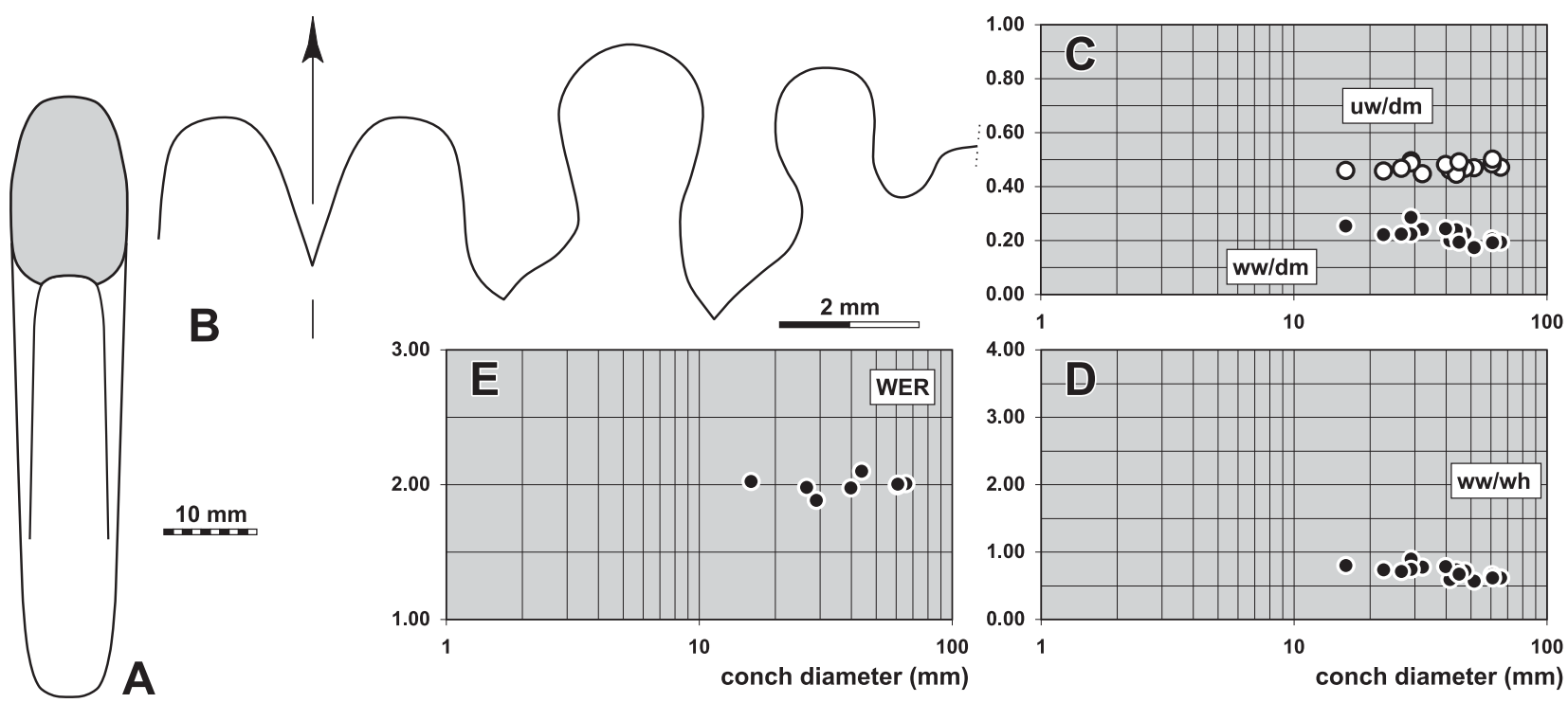

Figure 44. Merocanites merocanites n. sp. from locality TIM-C7. A. Dorsal view of holotype MB.C.18616.1; $\times 1.0$. B. Suture line of paratype MB.C.18616.3, at $23.0 \mathrm{~mm} \mathrm{dm}, 8.0 \mathrm{~mm}$ wh; $\times 7.5$. C-E. Ontogenetic development of the conch width index (ww/dm), umbilical width index (uw/dm), whorl width index (ww/wh), and whorl expansion rate (WER) of all available specimens. 


\section{Acknowledgements}

We are indebted to the regional Algerian authorities for their permission for us to undertake field work. D.K. acknowledges the Deutsche Forschungsgemeinschaft (DFG) for financial support (project Ko1829/3-1). Technical support was provided by Wolfgang Gerber (Tübingen) by photographing the specimens and by Evelin Stenzel and Markus Brinkmann (Berlin) for preparing of the material. We also thank Sonny Walton (Potsdam) for proofreading the manuscript and R. Thomas Becker (Münster) as well as Christian Klug (Zürich) for reviewing the article.

\section{References}

Barrois, C. 1879. Le marbre griotte des Pyrénées. - Annales de la Société Géologique du Nord 6: 270-300.

Barrois, C. 1882. Recherches sur les terrains anciens des Asturies et de la Galice. - Mémoires de la Société Géologique du Nord 2 (1): 1-630.

Bockwinkel, J., Korn, D. \& Ebbighausen, V. 2010. The ammonoids from the Argiles de Timimoun of Timimoun (Early and Middle Viséan; Gourara, Algeria). - Fossil Record 13 (1): 215-278.

Böhm, R. 1935. Études sur les faunes du Dévonien Supérieur et du Carbonifère Inférieur de la Montagne Noire. Charité, Montpellier: 203 pp.

Campbell, K. S. W., Brown, D. A. \& Coleman, A. R. 1983. Ammonoids and the correlation of the lower Carboniferous rocks of eastern Australia. - Alcheringa 7 (2): 75-123.

Chao, King-Koo \& Liang Xiluo 1964. Middle Carboniferous Cephalopoda. Handbook of Index fossils from Southern China: p. 87.

Collinson, C. 1955. Mississippian prolecanitid goniatites from Illinois and adjacent areas. - Journal of Paleontology 29 (3): 433-438.

Conrad, J. 1984. Les séries Carbonifères du Sahara Central Algérien stratigraphie, sédimentation, évolution structurale. Université de Droit, d'Economie et des Sciences d'Aix Marseille, Thèse de Doctorat d'Etat dès-Sciences naturelles. Marseille: 370 pp.

Crick, G. C. 1899. On some new or little-known Goniatites from the Carboniferous limestone of Ireland. - The annals and magazine of natural history, zoology, botany and geology (7) 3: 429-454.

Czarniecki, S. 1973. Goniatyty wapienia weglowego z Galezic. Rocznik Polskiego Towarzystwa Geologicznego 43: 227-248.

Delépine, G. 1940. Les goniatites du Dinantien de la Belgique. Mémoires du Museé Royal d'Histoire Naturelle de Belgique 91: $1-91$

Delépine, G. 1941. Les goniatites du Carbonifère du Maroc et des confins Algéro-Marocains du sud (Dinantien-Westphalien). Notes et mémoires, Service Géologique, Protectorat de la République Française au Maroc, Direction de la Production Industrielle et des Mines, Division des Mines et de la Géologie 56: 1-111.

Delépine, G. 1943. Les faunes marines du Carbonifère des Asturies (Espagne). - Mémoires de l'Académie des Sciences de l'Institut de France 66: 1-122.

Devuyst, F. X., Hance, L., Hou, H. F., Wu, X. H., Tian, S. G., Coen, M. \& Sevastopulo, G. 2003. A proposed global stratotype section and point for the base of the Visean stage (Carboniferous): the Pengchong section, Guangxi, South China. - Episodes 26 (2): $105-115$.

Dzik, J. 1997. Emergence and succession of Carboniferous conodont and ammonoid communities in the Polish part of the Variscan sea. - Acta Palaeontologica Polonica 42: 57-170.

Ebbighausen, V., Bockwinkel, J., Korn, D. \& Weyer, D. 2004. Early Tournaisian ammonoids from Timimoun (Gourara, Algeria). Mitteilungen aus dem Museum für Naturkunde Berlin, Geowissenschaftliche Reihe 7: 133-152.
Foord, A. H. \& Crick, G. C. 1897. Catalogue of the fossil Cephalopoda in the British Museum (Natural History), Part III, Containing the Bactritidae and part of the suborder Ammonoidea. London: $303 \mathrm{pp}$,

Foord, A. H. 1901. Monograph of the Carboniferous Cephalopoda of Ireland, Part IV, Containing the families Solenocheilidae (concluded) and Glyphioceratidae. - Palaeontographical Society 55: 127-146.

Foord, A. H. 1903. Monograph of the Carboniferous Cephalopoda of Ireland, Part V, Containing the families Glyphioceratidae (concluded) and Prolecanitidae, with title-page and index. - Palaeontographical Society 57: 147-234.

Frech, F. 1899. Lethaea geognostica, Teil 1: Lethaea palaeozoica, Band 2, Lieferung 2: Die Steinkohlenformation: 257-452.

Frech, F. 1902. Über devonische Ammoneen. - Beiträge zur Paläontologie Österreich-Ungarns und des Orients 14: 27-112.

Gischler, E. 1992. Das devonische Atoll von Iberg und Winterberg im Harz nach Ende des Riffwachstums. - Geologisches Jahrbuch A 129: 1-192.

Gordon, M. jr. 1957. Missisippian Cephalopods of Northern and Eastern Alaska. - Professional Papers of the United States Geological Survey 283: 1-61.

Gordon, M. Jr. 1965. Carboniferous Cephalopods of Arkansas. - Professional Papers of the United States Geological Survey 460: 1322 .

Gordon, M. Jr. 1986. Late Kinderhookian (Early Mississippian) Ammonoids of the Western United States. - Journal of Paleontology, Memoirs 19: 1-36.

Hall, J. 1860. Notes and observations upon fossils of the Goniatite Limestone in the Marcellus shale of the Hamilton group, in the eastern and central parts of the State of New York, and those of the Goniatite beds of Rockford, Indiana; with some analagous forms from the Hamilton group proper. - Annual Reports of the Regents of the University of the State New York, on the condition of the State Cabinet of Natural History, and the Historical and Antiquarian Collection annexed thereto 13: 59-112, 125.

Higgins, A. C. \& Wagner-Gentis, C. H. T. 1982. Conodonts, goniatites and the biostratigraphy of the earlier Carboniferous from the Cantabrian Mountains, Spain. - Palaeontology 25 (2): 313-350.

Higgins, A. C., Wagner-Gentis, C. H. T. \& Wagner, R. H. 1964. Basal Carboniferous Strata in Part of Northern León, NW. Spain: Stratigraphy, Conodont and Goniatite Faunas. - Bulletin de la Société Belge de Géologie, de Paléontologie et d'Hydrologie 72 (2): 205249

Hind, W. 1910. On four new Carboniferous nautiloids and a goniatite new to Great Britain. - Proceedings of the Yorkshire Geological Society 17: 97-109.

Holzapfel, E. 1889. Die Cephalopoden-führenden Kalke des unteren Carbon von Erdbach-Breitscheid bei Herborn. - Palaeontologische Abhandlungen, Neue Folge 5 (1): 1-74.

Hyatt, A. 1883-1884. Genera of fossil cephalopods. - Proceedings of the Boston Society of Natural History 22: 253-338 (253-272 publ. 1883, 273-338 publ. 1884).

Hyatt, A. 1900. Tetrabranchiate Cephalopoda. In Zittel, K. v. (ed.; transl. \& ed. by C. R. Eastman). Text-book of palaeontology, volume 1, 1st edition. McMillan, New York: pp. 502-604.

Koninck, L. G. de 1844. Description des animaux fossiles qui se trouvent dans le terrain carbonifère de la Belgique: 650 pp.; Liège.

Koninck, L. G. de 1880. Faune du Calcaire Carbonifère de la Belgique, deuxième partie, Genres Gyroceras, Cyrtoceras, Gomphoceras, Orthoceras, Subclymenia et Goniatites. - Annales du Musée Royal d'Histoire Naturelle de Belgique 5: 1-333.

Konovalova, V. A. 2005. Ammonoid Evolution at the Tournaisian-Visean Boundary. - Paleonotological Journal 39, Suppl. 5: S538S557.

Korn, D. 1997. Evolution of the Goniatitaceae and Viséan-Namurian biogeography. - Acta Palaeontologica Polonica 42: 177-199. 
Korn, D. 2010. A key for the description of Palaeozoic ammonoids. Fossil Record 13 (1): 5-12.

Korn, D. \& Ebbighausen, V. 2008. The Early Carboniferous (Mississippian) ammonoids from the Chebket el Hamra (Jerada Basin, Morocco). - Fossil Record 11 (2): 83-156.

Korn, D., Belka, Z., Fröhlich, S., Rücklin, M. \& Wendt, J. 2004. The youngest African clymeniids (Ammonoidea, Late Devonian) failed survivors of the Hangenberg Event. - Lethaia 37: 307315 .

Korn, D., Bockwinkel, J. \& Ebbighausen, V. 2007. The Tournaisian and Viséan ammonoid stratigraphy in North Africa. - Neues Jahrbuch für Geologie und Paläontologie 243 (2): 127-148.

Korn, D., Bockwinkel, J. \& Ebbighausen, V. 2010a: The ammonoids from the Argiles de Teguentour of the Mouydir (Late Tournaisian; Central Sahara, Algeria). - Fossil Record 13 (1): 35-152.

Korn, D., Bockwinkel, J., Ebbighausen, V. \& Klug, C. 2003a. Palaeobiogeographic and evolutionary meaning of an early Late Tournaisian ammonoid fauna from the Tafilalt of Morocco. - Acta Palaeontologica Polonica 48 (1): 71-92.

Korn, D., Ebbighausen, V. \& Bockwinkel, J. 2010b. Ammonoids from the Dalle des Iridet of the Mouydir and Ahnet (Central Sahara) and the Formation d'Hassi Sguilma of the Saoura Valley (Late Tournaisian-Early Viséan; Algeria). - Fossil Record 13 (1): 203214

Korn, D., Ebbighausen, V. \& Bockwinkel, J. 2010c. The ammonoids from the Grès du Kahla supérieur of Timimoun (Middle-early Late Tournaisian; Gourara, Algeria). - Fossil Record 13 (1): $13-$ 34.

Korn, D., Ebbighausen, V., Bockwinkel, J. \& Klug, C. 2003b. The Amode sutural ontogeny in prolecanitid ammonoids. - Palaeontology 46 (6): 1123-1132.

Korn, D. \& Feist, R. 2007. Early Carboniferous ammonoid faunas and stratigraphy of the Montagne Noire (France). - Fossil Record $10(2)$ : 99-124.

Korn, D. \& Ilg, A. 2009. AMMON. Database of Palaeozoic Ammonoidea. - http://www.wahre-staerke.com/ammon/

Korn, D., Klug, C. \& Mapes, R. H. 1999. Viséan and Early Namurian Ammonoids from the Tafilalt (Eastern Anti-Atlas, Morocco). Abhandlungen der Geologischen Bundesanstalt 54: 345-375.

Korn, D., Klug, C. \& Mapes, R. H. 2005. The Lazarus ammonoid family Goniatitidae, the tetrangularly coiled Entogonitidae, and Mississippian biogeography. - Journal of Paleontology 79 (2): $356-365$.

Krebs, W. 1968. Die Lagerungsverhältnisse des Erdbacher Kalkes (Unterkarbon II) bei Langenaubach-Breitscheid (Rheinisches Schiefergebirge). - Geotektonische Forschungen 28: 72-103.

Kullmann, J. 1961. Die Goniatiten des Unterkarbons im Kantabrischen Gebirge (Nordspanien). I. Stratigraphie. Paläontologie der U.O. Goniatitina Hyatt. - Neues Jahrbuch für Geologie und Paläontologie, Abhandlungen 113 (3): 219-326.

Kullmann, J. 1963. Die Goniatiten des Unterkarbons im Kantabrischen Gebirge (Nordspanien). II. Paläontologie der U. O. Prolecanitina Miller \& Furnish. Die Altersstellung der Faunen. Neues Jahrbuch für Geologie und Paläontologie, Abhandlungen 116 (3): 269-32.

Kusina, L. F. 1971. O nekotorykh novykh i maloizvestnykh ranevizeyskikh (saurskikh) ammonoideyakh. - Paleontologicheskiy Zhurnal 1971 (1): 37-48.

Kusina, L. F. 1973. K revizii roda Muensteroceras. - Paleontologicheskiy Zhurnal 1973 (3): 14-25.

Kusina, L. F. 1974. Saurskiy kompleks ranekamennougolnykh ammonoidey. - Paleontologicheskiy Zhurnal 1974 (4): 18-31.

Kusina, L. F. 1980. Saurskie amonoidei. - Trudy Paleontologicheskogo Instituta Akademiya Nauk SSSR 181: 1-108

Kusina, L. F. 1983. O nekotorykh novykh rannekamenougolnykh ammonoideyakh Urala. - Paleontologicheskiy Zhurnal 1983: 9195 .
Kusina, L. F. 2000. Ammonoidei iz pogranichnykh Turneysko-Vizeyskikh otlozhenii. - Paleontologicheskiy Zhurnal 2000 (5): 16-24.

Kusina, L. F. \& Konovalova, V. A. 2004. Novye ammonoidei iz pogranichnykh Turneysko-Vizeyskikh otlozhenii na reke Kozhim (Pripolyanyi Ural). - Paleontologicheskiy Zhurnal 2004 (1): 10-18.

Kusina, L. F. \& Yatskov, S. V. 1987. Kamenougolnye ammonoidei milinkoy svity Novoy Zemli. - Doklady Moskovskaya obshchestva ispytatelei prirody, otdelenie geologii 62: 101-108.

Liang Xi-luo \& Wang Mingqian 1988. Carboniferous Cephalopods of Xinjiang, NW China. In Wiedmann, J. \& Kullmann, J. (eds). Cephalopods - Present and Past. Schweizerbart'sche Verlagsbuchhhandlung, Stuttgart: pp. 553-575.

Liang Xi-luo \& Wang Mingqian 1991. Carboniferous cephalopods of Xinjiang. - Paleontologica Sinica, new series B 180: 1-171.

Librovitch, L. S. 1927. Nizhnekamennougolnye golovonogie iz rayona ozera Son-kul (Tyan Shan). - Materialy po obschey i prikladnoy geologii, Geologichesky Komitet 74: 1-55.

Librovitch, L. S. 1940. Ammonoidea iz kamennougol'nykh otlozheniy Severnogo Kazakhstana [Carboniferous Ammonoids of North Kazakhstan]. - Paleontologiya SSSR 4 (9/1): VI + 392 pp.

M'Coy, F. 1844. A synopsis of the Carboniferous Fossils of Ireland VIII $+274 \mathrm{pp}$

M'Coy, F. \& Griffith, R. 1862. A synopsis of the characters of the Carboniferous Limestone fossils of Ireland. $271 \mathrm{pp}$.

Menchikoff, N. 1930. Recherches géologiques et morphologiques dans le nord du Sahara occidental. - Revue de Géographie physique et de Géologie dynamique 3 (2): 103-247.

Miller, A. K. 1931. Two new genera of Late Paleozoic cephalopods from Central Asia. -American Journal of Science, 4th series 522: 417-425.

Miller, A.K. \& Collinson, C. 1951. Lower Mississippian ammonoids of Missouri. - Journal of Paleontology 25: 454-487.

Miller, A. K. \& Furnish, W. M. 1958. Goniatites of the Burlington Limestone of Missouri. - Journal of Paleontology 32 (2): 269274.

Miller, A. K. \& Garner, H. F. 1955. Lower Mississippian cephalopods of Michigan. Part III. Ammonoids and summary. - Contributions of the Museum of Paleontology, University of Michigan 12: 113173.

Miller, A. K. \& Werner, C. 1942. A goniatite from the Mississippian Fern Glen formation of Illinois. - Journal of Paleontology 16 : 479-481.

Miller, A. K., Downs, H. R. \& Youngquist, W. 1949. Some Mississippian cephalopods from central and western United States. - Journal of Paleontology23: 600-612.

Müller, K. J. 1959. Nachweis der Pericyclus-Stufe (Unterkarbon) in den Karnischen Alpen. - Neues Jahrbuch für Geologie und Paläontologie, Abhandlungen 1959: 90-94.

Pardo Alonso, M. A. \& Kullmann, J. 2002. Erster AmmonoideenFund (Cephalopoda) im Unterkarbon der Zentral-Iberischen Zone in der Sierra Morena (Süd-Spanien). - Neues Jahrbuch für Geologie und Paläontologie, Monatshefte 2002: 597-608.

Pareyn, C. 1961. Les Massifs Carbonifères du Sahara Sud-Oranais Tome II. Paléontologie stratigraphique. - Publications du Centre de Recherches Sahariennes, Série Géologie 1: 1-244.

Parkinson, J. 1822. Introduction to the study of fossil organic remains I. Sherwood, Neely and. Jones, London: VII + 346 pp.

Petersen, M. S., Korn, D. \& Kullmann, J. 2000. The Early Mississippian (Osagean) ammonoid Dzhaprakoceras (Cephalopoda) from Utah. - Journal of Paleontology 74 (5): 853-857.

Phillips, J. 1836. Illustrations of the geology of Yorkshire; or a description of the strata and organic remains; accompanied by a geological map, sections, and diagrams, and figures of the fossils. Part II. The Mountain Limestone District. London: $\mathrm{XX}+259 \mathrm{pp}$

Popov, A. V. 1965. Novye vizeyskie ammonoidei Tyan-Shanya. - Paleontologicheskiy Zhurnal 1965 (2): 35-49. 
Popov, A. V. 1968. Vizeyskie ammonoidei cevernogo Tyan-Shanya i ikh stratigraficheskoe zhachenie. - Trudy Geologicheskiy Instituta, Kirgizskiy Filial Akademiya Nauk SSSR: 1-116.

Popov, A. V. 1975. Ammonoidea (Ammonoidei). In Paleontologicheskii Atlas kamennougol'nykh otlozhenii Urala. Trudy Vsesoyuznyy Nauchno-Issledovatel'skiy Geologo-Razvedochnyy Neftyanoy Institut 383: 111-130.

Popov, A. V. \& Kusina, L. F. 1997. The earliest Goniatitina (Ammonoidea) from the South Urals. - Paleontological Journal 31: 2834.

Riley, N. J. 1990. A global review of Mid-Dinantian ammonoid biostratigraphy. - Courier Forschungsinstitut Senckenberg 130: 133143.

Riley, N. J. 1996. Mid-Dinantian ammonoids from the Craven Basin, northwest England. - Special papers in Palaeontology 53: 1-87.

Roemer, F. A. 1852. Beiträge zur geologischen Kenntniss des nordwestlichen Harzgebirges. Zweite Abtheilung. - Palaeontographica 3: $69-111$

Schindewolf, O. H. 1951. Über ein neues Vorkommen unterkarbonischer Pericyclus-Schichten im Oberharz. - Neues Jahrbuch für Geologie und Paläontologie, Abhandlungen 93 (1): 23-116.

Schmidt, H. 1925. Die carbonischen Goniatiten Deutschlands. - Jahrbuch der Preußischen Geologischen Landesanstalt 45 (for 1924): 489-609.

Schmidt, H. 1931. Das Paläozoikum der spanischen Pyrenäen. - Abhandlungen der Gesellschaft der Wissenschaften in Göttingen, Mathematisch-Physikalische Klasse III (3) 5: 1-85.

Sheng Huaibin 1984. Lower Carboniferous ammonoid faunule from the Zhifang area, Xinjiang. - Acta Geologica Sinica 1984 (4): 284-292.

Shimansky, V. N. \& Kusina, L. F. 1977. Rannekamennougolnye golovonogie pripolyatnogo Urala. - Byulleten' Moskovskogo Obshchestva Ispytateley Prirody, Otdel Geologicheskiy 52 (6): 7990 .

Smyth, L. B. 1951. A Viséan cephalopod fauna in the Rush Slates of Co. Dublin. - Proceedings of the Royal Irish Academy B 53: 289-309.

Sowerby, J. 1812-1814. Mineral Conchology of Great Britain, volume I. 234 pp.
Sowerby, J. 1820. Mineral Conchology of Great Britain, volume III. 194 pp.

Swallow, G. C. 1860. Descriptions of new fossils from the Carboniferous and Devonian rocks of Missouri. - Transactions of the St. Louis Academy of Science 1: 635-660.

Tilsley, J. W. \& Korn, D. 2009. Chadian (Tournaisian-Viséan; Carboniferous) ammonoids from the Milldale Limestone Formation of the southern Peak District, England. - Proceedings of the Yorkshire Geological Society 57 (3/4): 133-150.

Turner, J. S. 1948. Mid-Dinantian reef limestones of Dublin and Cork. - Transactions of the Leeds Geological Association 6 (2): 44-56.

Walliser, O. H. 1966. Preliminary notes on Devonian, Lower and Upper Carboniferous goniatites in Iran. - Report, Geological Survey of Iran 6: 7-24.

Wendt, J., Kaufmann, B., Belka, Z. \& Korn, D. 2009. Carboniferous stratigraphy and depositional environments in the Ahnet Mouydir area (Algerian Sahara). - Facies 55 (3): 443-472.

Weyer, D. 1972. Trilobiten und Ammonoideen aus der Entogonites nasutus-Zone (Unterkarbon) des Büchenberg-Sattels (Elbingeröder Komplex, Harz). Teil I. - Geologie 21 (2): 166-184.

Winchell Winchell, A. 1862. Notice of the rocks lying between the Carboniferous limestone of the Lower Peninsula of Michigan and the limestones of the Hamilton group; with descriptions of some cephalopods supposed to be new to science. - American Journal of Science and Arts 33: 352-366.

Work, D. M. \& Mason, C. E. 2003. Mississippian (middle Osagean) ammonoids from the Nada Member of the Borden Formation, Kentucky. - Journal of Paleontology 77 (3): 593-596.

Work, D. M. \& Mason, C. E. 2004. Mississippian (late Osagean) ammonoids from the New Providence Shale Member of the Borden Formation, north-central Kentucky. - Journal of Paleontology 78 (6): 1128-1137.

Work, D. M. \& Mason, C. E. 2005. Mississippian (early Osagean) Cave Run Lake ammonoid fauna, Borden Formation, northeastern Kentucky. - Journal of Paleontology 79 (4): 719-725.

Work, D. M., Nassichuk, W. W. \& Richards, B. C. 2000. Lower Viséan ammonoids from the Lower Mount Head Formation, East-Central British Columbia. - Bulletin of the Geological Survey of Canada 541: 1-71. 